\title{
Spectrum Inference in Cognitive Radio Networks: Algorithms and Applications
}

\author{
Guoru Ding, Senior Member, IEEE, Yutao Jiao, Jinlong Wang, Senior Member, IEEE, \\ Yulong Zou, Senior Member, IEEE, Qihui Wu, Senior Member, IEEE, \\ Yu-Dong Yao, Fellow, IEEE, and Lajos Hanzo, Fellow, IEEE
}

\begin{abstract}
Spectrum inference, also known as spectrum prediction in the literature, is a promising technique of inferring the occupied/free state of radio spectrum from already known/measured spectrum occupancy statistics by effectively exploiting the inherent correlations among them. In the past few years, spectrum inference has gained increasing attention owing to its wide applications in cognitive radio networks (CRNs), ranging from adaptive spectrum sensing, and predictive spectrum mobility, to dynamic spectrum access and smart topology control, to name just a few. In this paper, we provide a comprehensive survey and tutorial on the recent advances in spectrum inference. Specifically, we first present the preliminaries of spectrum inference, including the sources of spectrum occupancy statistics, the models of spectrum usage, and characterize the predictability of spectrum state evolution. By introducing the taxonomy of spectrum inference from a time-frequency-space perspective, we offer an in-depth tutorial on the existing algorithms. Furthermore, we provide a comparative analysis of various spectrum inference algorithms and discuss the metrics of evaluating the efficiency of spectrum inference. We also portray the various potential applications of spectrum inference in CRNs and beyond, with an outlook to the 5th generation mobile communications (5G) and next generation high frequency (HF) communications systems. Last but not least, we highlight the critical research challenges and open issues ahead.
\end{abstract}

The work of G. Ding, J. Wang, and Q. Wu is supported in part by the National Natural Science Foundation of China under Grants 61501510 and 61631020, Natural Science Foundation of Jiangsu Province under Grant BK20150717, China Postdoctoral Science Funded Project, and Jiangsu Planned Projects for Postdoctoral Research Funds. The work of Y. Zou is supported in part by the National Natural Science Foundation of China under Grants 61401223 and 61522109 and the Natural Science Foundation of Jiangsu Province under Grants BK20140887 and BK20150040. The work of L. Hanzo is supported in part by the European Research Council's Advance Fellow Grant Beam-Me-Up and the Royal Society's Wolfson Research Merit Award. Y. Jiao and L. Hanzo are the corresponding authors.

G. Ding and J. Wang are with the College of Communications Engineering, PLA University of Science and Technology, Nanjing 210007, China (email: dr.guoru.ding@ieee.org; wj1543@sina.com). G. Ding is also with the National Mobile Communications Research Laboratory, Southeast University, Nanjing 210096, China.

Y. Jiao is with the School of Computer Science and Engineering, Nanyang Technological University, Singapore 639798 (email: yjiao001@ ntu.edu.sg). This work was done while he was at Nanjing University of Posts and Telecommunications, Nanjing 210003, China.

Y. Zou is with the School of Telecommunications and Information Engineering, Nanjing University of Posts and Telecommunications, Nanjing 210003, China (email: Yulong.Zou@ njupt.edu.cn).

Q. Wu is with the College of Electronic and Information Engineering, Nanjing University of Aeronautics and Astronautics, Nanjing, 211106, China (email: wuqihui2014@sina.com)

Y. Yao is with the Department of Electrical and Computer Engineering, Stevens Institute of Technology, Hoboken, NJ 07030, USA (email: yyao@stevens.edu), and Northeast University, Shenyang, China.

L. Hanzo is with the School of Electronics and Computer Science, University of Southampton, Southampton, UK (email: 1h@ecs.soton.ac.uk).
Index Terms-Spectrum inference, spectrum prediction, cognitive radio, 5G, HF communications

\section{INTRODUCTION}

\section{A. Background and Motivation}

The contradiction between spectrum shortage and spectrum under-utilization has motivated the emergence of dynamic spectrum access (DSA) or opportunistic spectrum access (OSA) [1]. Cognitive radio (CR) [2] has been well recognized as one of the crucial techniques of realizing the DSA/OSA concept. Since its conception, CR has been designed for autonomous reconfiguration by learning from and ultimately adapting to the continuously changing radio environment [3].

The first step of implementing a $\mathrm{CR}$ is to capture the relevant information about the spectral evolution. In the spectrum management framework relying on spectrum sensing, spectrum allocation decisions, spectrum sharing, and spectrum mobility proposed in [4], spectrum sensing has the task of sensing its occupancy and capturing the characteristics of the primary user (PU). However, in the practical sensing process, some inevitable problems arise concerning the sensing speed, the potentially excessive energy consumption and the limited sensing scope, all of which hinder the efficient operation of the $\mathrm{CR}$. The main reason for these problems is that each CR can only sense the current radio environment at its operating location without any awareness of the unsensed bands or locations and of the future trends of the spectral domain activities [5]. This inevitably wastes precious information about the evolution of spectral states between time slots, frequency bands, geographical locations, etc.

Spectrum inference/prediction is known as an effective technique complementary to spectrum sensing for capturing the relevant information about the spectral evolution and identifying spectrum holes. Briefly, spectrum sensing determines the spectrum state in a passive manner using various signal detection methods. By contrast, spectrum inference/prediction is a promising technique of inferring the occupied/free state of radio spectrum from already known/measured spectrum occupancy statistics by effectively exploiting the inherent correlations among them, in a proactive manner. Fortunately, some other fields of application, such as the atmosphere [6], finance [7], network traffic [8] and human mobility [9], inference/prediction techniques (including the popular big data technique [10]) have provided potential techniques of discovering the usage patterns hidden in the data and have 


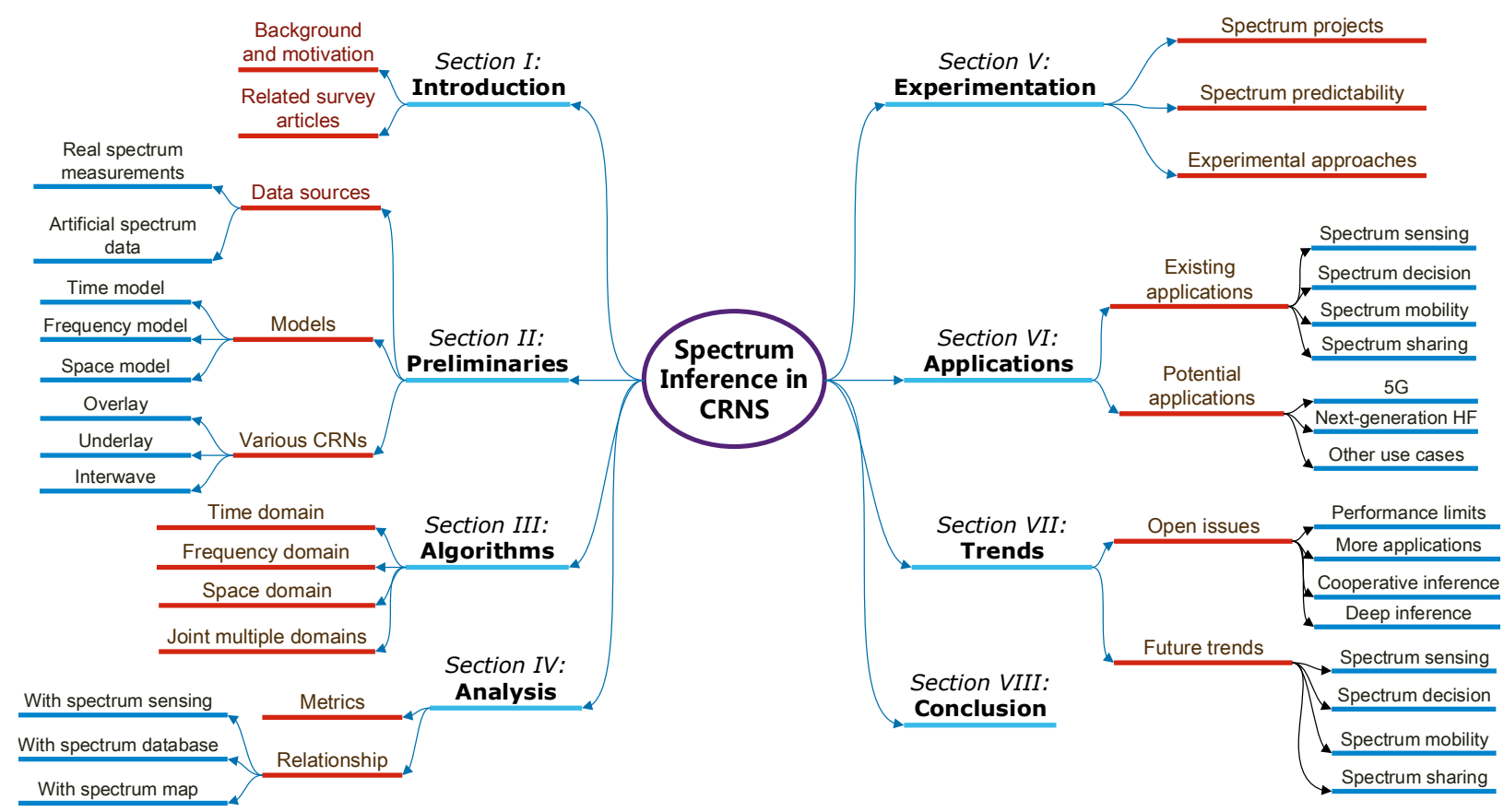

Fig. 1: The structure of this paper.

succeeded in maintaining the stability of the economy, forecasting the weather conditions and so on. Similarly, spectrum inference/prediction is a promising technique that can be utilized for acquiring precious unknown spectrum occupancy information in advance and for enhancing the performance of the CRs by accelerating the process of choosing the best channel and expanding the sensing scope in the timefrequency-spatial-domain [11]-[13]. Therefore, an increasing number of researchers have been focusing on using or developing effective inference/prediction techniques for CRs. Preliminary results (see, e.g., [14]-[20]) demonstrate that spectrum inference/prediction algorithms operating across the time-frequency-spatial dimensions may equip CRs with an accurate forecasting capability. Consequently, there is an urgent need for a survey of the specific algorithms, since the inference/prediction function is at the heart of the CR architecture and operates in a realm, where communications techniques meet artificial intelligence.

\section{B. Comparison with Related Survey Articles}

Historically speaking, there is only a brief survey relying on 15 citations [21] on spectrum prediction in CRNs, which is focused on a temporal scope. By contrast, the goal of this paper is twofold. The first is to present an all-encompassing systematic survey of the recent advances in spectrum inference/prediction algorithms from a time-frequency-spatial domain perspective. The second is to discuss the potential applications and prospects in order to emphasize the significance of inference/predicton techniques in the context of the CR technology and to provide guidelines for researchers focusing on making CR more intelligent and efficient.

Notably, spectrum inference is also related to the subject of spectrum occupancy measurements [23], [24] and primary user activity modeling [22]. Briefly, primary user activity models use mathematical or theoretical expressions to mimic the underlying PU activities, while spectrum inference algorithms use machine learning or data mining methods to predict or infer the future or unknown PU activities based on the available historic data. Refs. [22]-[24] are excellent survey and tutorial papers on spectrum occupancy measurements and/or theoretical modeling relying on statistical analysis, while our paper focuses on presenting various data mining algorithms conceived for spectrum inference/prediction. Indeed, the spectrum models in Refs. [22]-[24] are very useful for theoretical performance analysis or simulated data generation. However, they cannot be directly used for prediction.

Moreover, the work of this survey paper is also related to the survey papers on various aspects of CRNs, such as spectrum sensing [25]-[30], spectrum decision [31]-[35], resource allocation [36]-[41], security and privacy [42]-[48], MAC protocols [49], [50], routing [51], network coding [52], various applications [53]-[56], to just mention a few. A list of the related survey papers is provided in Table I for further references. On one hand, the studies on spectrum inference can find their applications in these aspects, which will be discussed in detail in the following sections. On the other hand, spectrum inference to some extent is a parallel or complementary technique to those in Table I. This paper fills the gap between them by providing a comprehensive survey of spectrum inference in CRNs.

\section{Organization and Notation}

This paper is organized as illustrated in Fig. 1. We introduce the necessary preliminary knowledge on spectrum inference in Section II. Specific inference algorithms operating in the timefrequency-spatial domain are detailed in Section III, while 
TABLE I: A List of Related Survey Articles

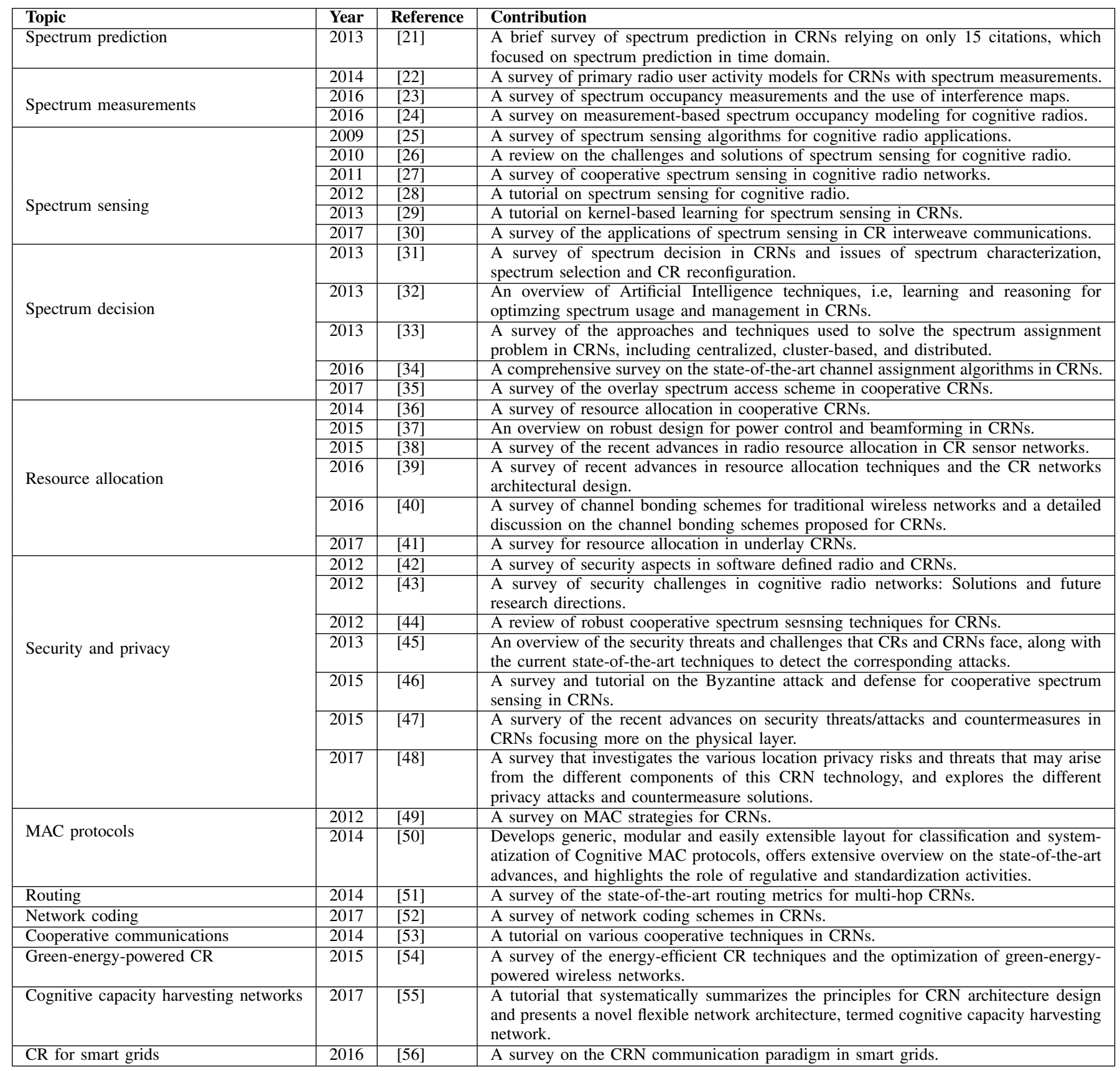

Section IV proposes a macroscopic view of various inference methods. The current and potential future applications are introduced in Section V. Finally, a range of challenges and future trends are presented in Section VI along with our conclusions. The acronyms used in this article can be found in the Table VII for convenience.

\section{PRELIMINARIES FOR SPECTRUM INFERENCE IN CRNS}

\section{A. Sources of Spectrum Data}

Empirical real world spectrum measurements constitute the very important source of spectrum data and play a fundamental role in supporting the research and development of spectrum inference techniques. Numerous spectrum occupancy measurement campaigns have been conducted all over the world (see, e.g., [23], [24], [59]-[87]). Excellent surveys and tutorials on the latest advances of worldwide spectrum occupancy measurements can be found in [23] and [24]. Briefly, here we highlight some common features of the spectrum measurement campaigns as follows.

Firstly, the campaigns have covered various frequency ranges. For example, the probably earliest measurement campaigns conducted by the Institute of Telecommunication Science in the USA around 1995 [59] measured very broad frequency bands spanning from $108 \mathrm{MHz}$ to $19.3 \mathrm{GHz}$. One the other hand, the very recent ones carried out in Beijing [86] 


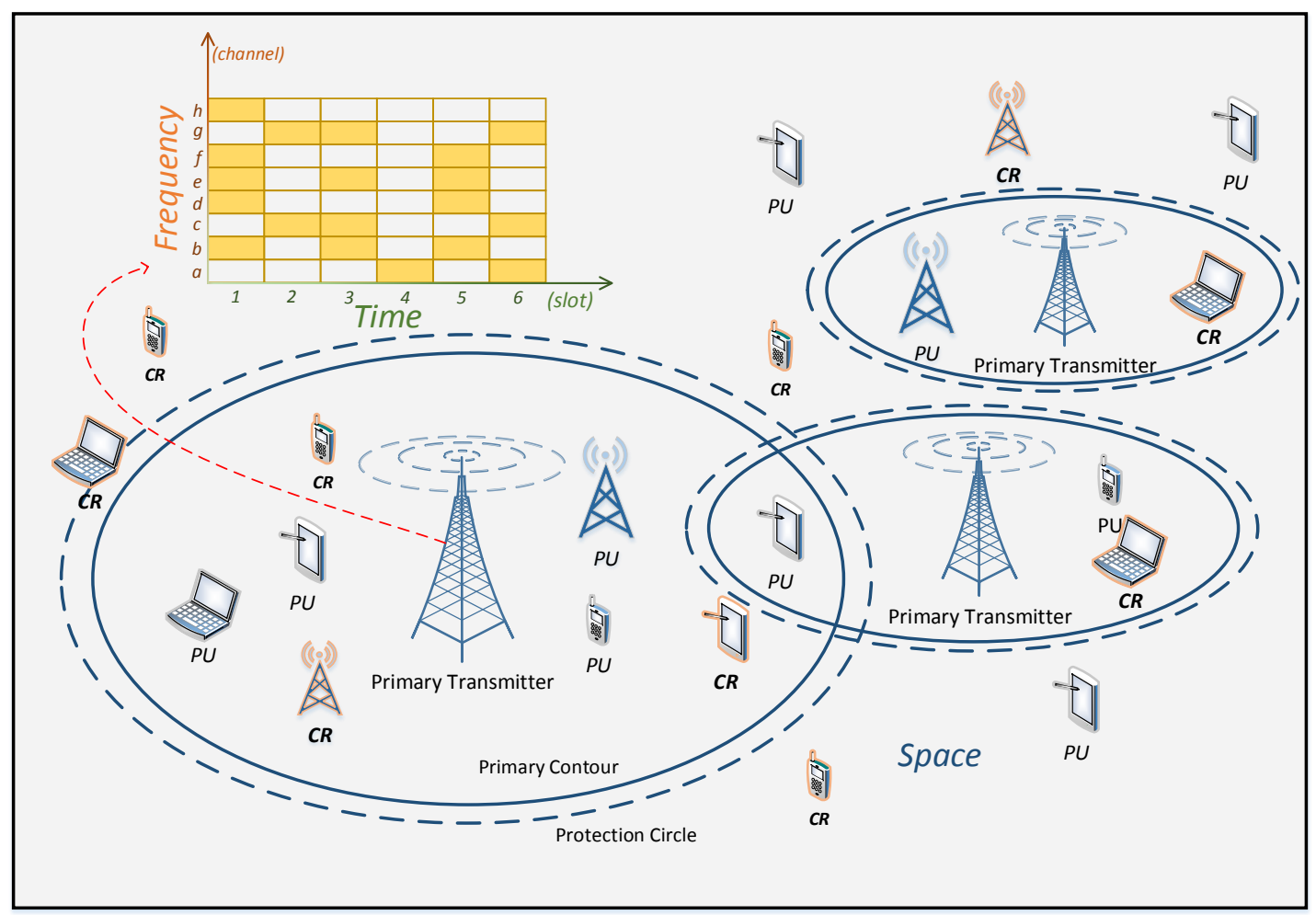

Fig. 2: An illustrative scenario in the time-frequency-spatial dimension.

and London [87] focused on TV bands (e.g., $470 \mathrm{MHz} \sim 790$ $\mathrm{MHz}$ ) to identify TV white spaces. Moreover, there are many other campaigns that measured specific licensed bands (like WiFi bands, TV bands, radar bands and cellular bands) relying on diverse lengths of measurement periods (from a few hours to several years) as well as different places (e.g., Denver, Dublin, Aachen, and Singapore, etc) and scenarios (including rural, suburban and urban areas, indoor and outdoor cases) [23], [24], [59]-[87].

Second, the campaigns have had various measurement setups. In terms of hardware devices, the spectrum measurement systems generally consist of the antenna, the filter, the amplifier and the spectrum analyser to collect the spectrum data. Most of the spectrum measurement campaigns specify several common setups. For example, both the sampling rate and the measurement period, as well as the frequency span and frequency points, the measurement location, antenna polarization and direction have to be chosen according to the particular applications considered. Moreover, since the various settings may lead to different levels of measurement complexity and accuracy, the tradeoffs among of these potentially conflicting factors have been carefully taken into consideration.

Third, the campaigns have a tendency to bridge real world spectrum measurements and the public/private spectrum databases [247]-[250]. On one hand, the collection, storage and evaluation of massive amounts of spectrum measurements requires advanced databases. On the other hand, spectrum measurements ensures a data-driven approach, complementarily to the traditional propagation model-based approach [242], when aiming for improving the accuracy and the update speed of the spectrum availability provided by the geoloca- tion spectrum databases [5], [88], [246]. Moreover, spectrum measurements are also coupled with the building of radio environment maps (REM) [255]-[257].

\section{B. Models of Spectrum Usage}

Exploiting the statistics of spectrum use or the deterministic status of PUs have been one of the critical issues for secondary use of the licensed spectrum. The research on the PU's behavior gleans lessons from the spectrum measurement campaigns. A brief conclusion is that the obtained spectrum occupancy results, e.g., in terms of the duty cycle, from spectrum measurement campaigns run by different groups are not always the same at different measurement time, locations, frequency bands with various measurement hardware and softwares. However, the primary user occupancy models (e.g., DTMC and CTMC) discussed in the following are widely used by different measurement campaigns associated with various parameter setups for specific bands, locations, and time.

Specifically, in CRNs, spectrum usage models of the primary system usually determine both the action and the performance of a secondary network and thus they play a significant role in spectrum inference, since the exploration and evaluation of inference algorithms as well as techniques is often based on them [90]. Spectrum usage models can be utilized to discover patterns of the PU's activities by analyzing the spectrum data and by reconstructing the statistical properties of the spectrum usage in real radio communication systems. By taking advantage of these models, we can generate simulated data for validating the inference algorithms. In order to prepare a solid basis for our discussions in the next section, we will 


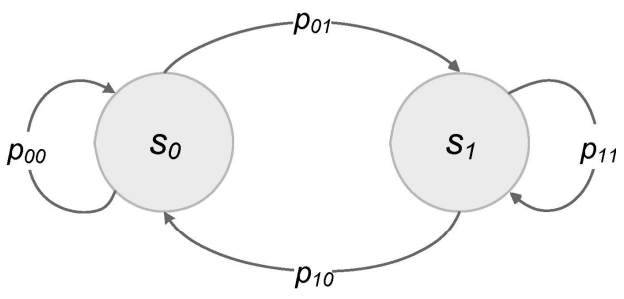

Fig. 3: DTMC model.

briefly present the most common approaches of modelling spectrum usage across the three orthogonal dimensions of time, frequency and space, as illustrated in Fig. 2 [19], [22], [89], [91]-[93]. Explicitly, in Fig. 2, there are several primary transmitters having their own primary coverage area, which are carefully coordinated for mitigating the interference arising from the others. A number of PUs and CRs are roaming either within or outside the coverage area of these primary transmitters. Furthermore, every primary transmitter has its spectrum for licensed or unlicensed use.

1) Time Domain Model: In the time domain, the spectrum usage may simply be modeled by a Markov chain having two states, one representing that the channel is busy and the other one representing that it is idle. In terms of temporal continuity, these models can be classified either into the discrete-time Markov chain (DTMC) model or into the continuous-time Markov chain (CTMC) model.

a) DTMC Model: Since the PU's states can be described as being either busy or idle and most measured data are usually represented in the form of binary sequences, the spectrum usage patterns are reflected by bits of zero value indicating having no traffic and logical ones indicating that the spectrum is being used by the PU at a particular time instant [94], [95]. The state space of a primary radio channel obeying the DTMC is denoted by $S=\{0,1\}$. The time index set is discrete, $t=t_{k}=k T_{s}$, where $k$ is a non-negative integer representing the step index and $T_{s}$ is the time period between consecutive transitions or state changes.

The most important parameter of the DTMC model is the transition probability $p_{i j}\left(t_{m}, t_{n}\right) \in\{0,1\}$, which represents the probability that channel state moves from state $i$ in the $m$ th slot to state $j$ in slot $n$. In this particular case, the transition matrix is given by:

$$
P=\left[\begin{array}{ll}
p_{00} & p_{01} \\
p_{10} & p_{11}
\end{array}\right] .
$$

The overall DTMC model describing the activity of a PU's channel is illustrated in Fig. 3. We can use the abovementioned concept of DC, denoted by $\Psi$, as a metric of describing the ratio of time during which the channel is declared busy as well as idle [89], [91].

In terms of stationarity, the DTMC models fall into two categories: stationary and non-stationary DTMC models. In the stationary DTMC model, the transition matrix can be formulated as,

$$
P=\left[\begin{array}{ll}
1-\Psi & \Psi \\
1-\Psi & \Psi
\end{array}\right]
$$

where $\Psi$ is a constant parameter and it can be selected as $\Psi=p_{01}=p_{11}$, especially in the long term $\Psi=P(s=1)$. In this way, the DTMC model becomes capable of reproducing the mean DC of a real channel. However, the stationary DTMC may only be valid for a limited time period, if the system exhibits only an approximately stationary behavior during this period, where the reproduced average DC approximately matches the instantaneous DC at all times [89].

If the modeled system does not appear to exhibit strictly stationary characteristics, a non-stationary DTMC model should be considered. In this case, the transition matrix should be defined as,

$$
P(t)=\left[\begin{array}{ll}
1-\Psi(t) & \Psi(t) \\
1-\Psi(t) & \Psi(t)
\end{array}\right],
$$

where $\Psi(t)$ is a variable, which is a function of the discrete time $t$. Based on different patterns of the channel's load, DC models can be developed using both deterministic and stochastic modelling approaches [89]. On one hand, in many particular services, like GSM and TETRA, common human habits and social behaviors impose a significant impact on the load patterns, which deterministically shape the characteristics of $\Psi(t)$. On the other hand, the stochastic DC modelling approaches are presented to describe the random variable $\Psi(t)$ as a function of numerous random factors contributing to the PU's behavior in real scenarios. It was found that the empirical PDFs of $\Psi(t)$ can be modelled by the Beta distribution [96] and the Kumaraswamy distribution [97].

Furthermore, extensive empirical measurement results have shown that the DTMCs associated with the deterministic and stochastic DC models mentioned above are capable of reproducing the average $\mathrm{DC}$ value and the statistical properties of the PU's idle or busy duration in real-world channels. The DTMCs have also been widely used in the literature to analyze the system throughput, the average packet delay and the endto-end packet delay (see, e.g. [98], [99]).

b) CTMC Model: In contrast to the DTMC model, the CTMC model pays closer attention to modeling the length of a state's holding time. Note that the traditional exponential distribution used for characterizing the state holding time in CTMC is not necessarily accurate enough, according to a range of realistic measurements and analysis conducted across the globe [91], [100]-[106]. In view of this, many researchers considered the Continuous-Time Semi-Markov Chain (CTSMC) models, where the sojourn time may obey arbitrary distributions. In [104], [105], [107], [108], the idle duration was assumed to obey either a generalized Pareto distribution [108], or a mixture of uniform distribution and the generalized Pareto distribution, or alternatively, obey a hyper-Erlang distribution [104], [105] and so on. In [89], the Lopez et al. employ goodness-of-fit metrics to evaluate several common distributions and to demonstrate that in real-world systems CTSMC models have a better performance in terms of reproducing the statistical properties of the busy and idle durations. Additionally, the correlation between the busy and idle durations, which the CTSMC fails to reproduce has been analyzed in [91], [109]. 
2) Frequency Domain Model: In this paper, the frequencydomain model can also be termed as the time-frequency model, since we do analyze the properties of spectrum data over the allocated frequency band based on the channel's DC which is a temporal parameter. Although the channels are considered to be mutually independent within a spectrum band [90], [110], this may not be the case for adjacent frequency-domain channels [92]. Hence, when modeling the spectrum usage in the frequency domain, the dependency among neighboring channels should be taken into consideration. With respect to the statistical correlation across the frequency band, two aspects can be investigated through the analysis of real-world spectrum data. The first one is the DC distribution within an allocated band, while the second is the cumulative distribution function (CDF) of the DC distribution, which are closely matched by the Beta distribution, by the Kumaraswamy distribution and so on [89].

Another aspect to consider is the DC clustering across the frequency bands. In [111], the similar DC values of the adjacent channels were classified into the same group, and the distribution of the size of the cluster group was described by an appropriately shifted version of the geometric distribution [96].

3) Spatial Domain Model: In recent years, numerous studies considered the relationships among the channel states observed by CRs in different scenarios [112]-[114]. Making use of the location information beneficially supports the CR's initial awareness of the environment and its ability of taking actions efficiently [115], [116]. Based on the above-mentioned DC models in the time- and frequency- domains, the spatial DC models and the modelling of simultaneous observations are briefly introduced below. Reference [89] discussed the spatial DC models under different conditions, namely when concerning either time-varying or constant power and continuous or discontinuous transmitters.

The DC values, $\Psi$, are calculated after the process of energy detection. The authors in [89] also discussed the conditional and the joint probabilities of the state observed at an arbitrary location and the simultaneously observed state at the reference location. Given these two probabilities, we will be able to model the spectrum usage at another location based on the model at the reference location. Therefore, the spatial modelling procedure attaches importance to the correlation of the spectrum usage at various locations for supporting spectrum inference/prediction. However, it should be noted that generally spectrum measurement campaigns are conducted by measuring the received signals and then estimating the status of the PUs using various spectrum sensing techniques, such as energy detection [117], matched filtering, and waveform based sensing. Due to the inevitable noise, incomplete observations, and the ambiguity of parameters like the energy decision threshold and the undiscovered spectrum features, such formulations are unable to accurately infer the PUs' true status and to capture the real characteristics of the PU's activity [117]-[119]. In this sense, all the models, including those introduced above may be imperfect, which necessitates further research.

\section{Spectrum Inference in Various CRNs}

Depending on the type of available side information and on the regulatory constraints, there are three main CRNs paradigms: underlay, overlay, and interweave [120]. Briefly, the underlay CRN allows CRs to operate if the interference they cause is below a given threshold. In overlay CRNs, the CRs overhear the transmissions of the PUs, and then use this information along with sophisticated signal processing techniques to improve the performance of PUs, while also obtaining some additional bandwidth for the CRs' own transmission. In interweave CRNs, the CRs sense the absence of a PU signal in the time, frequency, and/or spatial domains, and opportunistically communicate during the PUs' absence. Generally, underlay, overlay, and interweave can be used in different bands (e.g., TV white space, radar bands and cellular bands) according to the specific regulations in a separate or hybrid manner [121], [122].

Most of the existing studies on spectrum inference focus their attention on interweave CRNs, i.e., on predicting the spectrum occupancy state in terms of being idle (i.e. the absence of a PU signal) or occupied (i.e. the presence of a PU signal), by licensed users. There are relatively few studies on spectrum inference in other types of CRNs such as underlay, overlay and hybrid CRNs. It is an interesting and fruitful research direction to extend the research from interweave CRNs to other types of CRNs. Technically, a key difference is that the inference of binary spectrum state (i.e. idle or busy) in interweave CRNs should be extended to the inference of multilevel or even continuous spectrum state values in underlay and overlay CRNs, relying on the knowledge of the channel state information between PU transmitters and PU receivers.

\section{Spectrum Inference Algorithms in CRNs}

Numerous prediction algorithms have been proposed for forecasting the channel state, the spectrum occupancy, the potential interference imposed, the PU's coverage area and so on. In this section, the common spectrum inference/prediction algorithms found in the literature will be surveyed in the time, frequency and spatial domains by adopting a multidimensional approach. The taxonomy of these spectrum inference algorithms is illustrated in Fig. 4.

\section{A. Temporal Spectrum Inference Algorithms}

The three main branches of Fig. 4 will be surveyed in Subsections A, B and C respectively, commencing with the temporal techniques. In the time domain, spectrum prediction infers the status of spectrum according to the historical information on the evolution of the spectral occupancy [128]. The information gleaned is represented by a series of numbers extracted from the original data, with the aid of linear prediction, Markov modelling, neural networks, pattern mining, etc. After acquiring the regular patterns of the PU, we can sense the spectrum in less time than usual, conserve precious energy and make collisions among PUs and CRs more infrequent. 


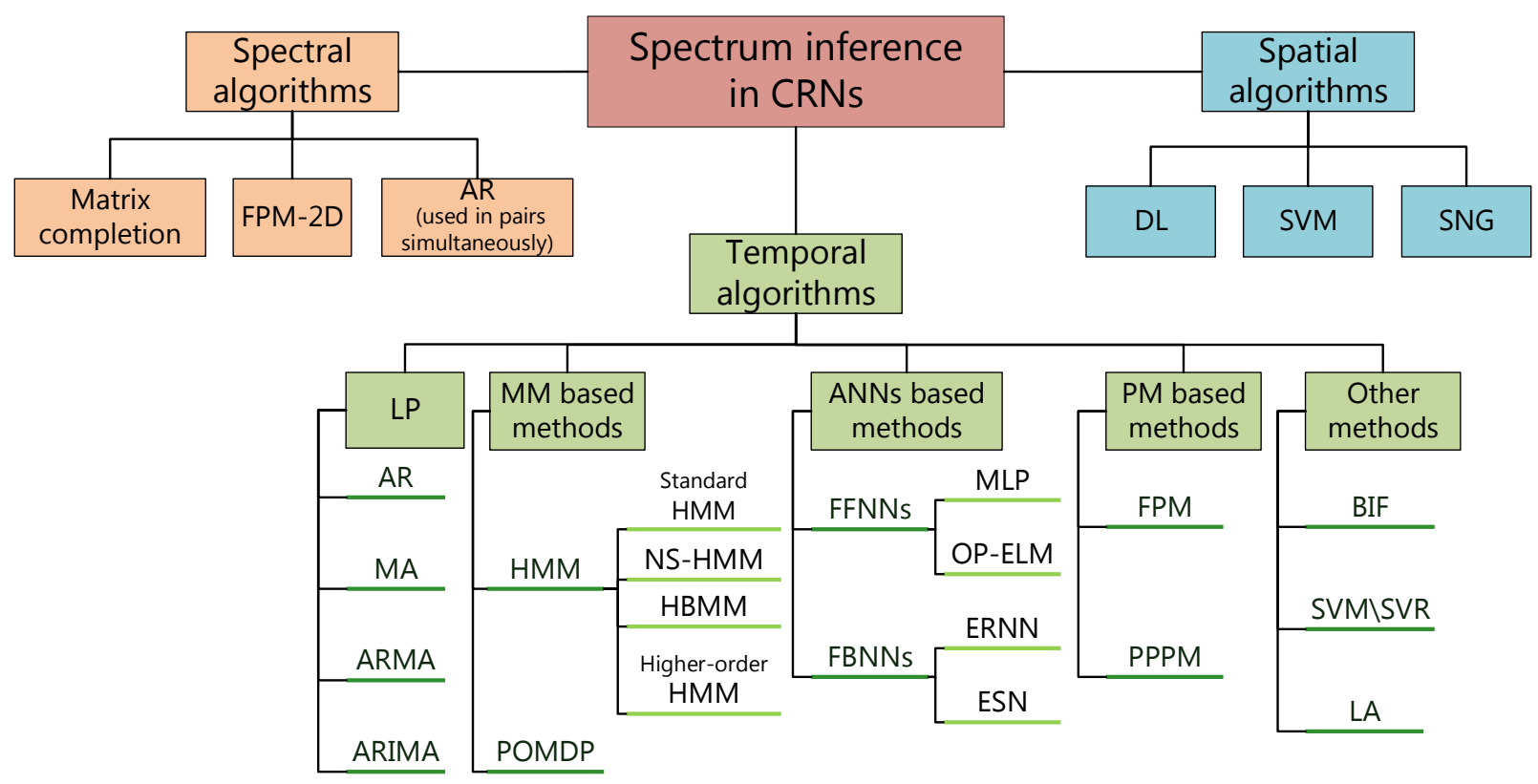

Fig. 4: Taxonomy of spectrum inference algorithms in the literature (See Table VII for the acronyms).

1) Linear Prediction (LP): Let us commence with LP algorithms seen at the top of the temporal branch. Linear prediction is an important branch of mathematical statistics, where future values are predicted as a linear function of previous samples. The LP based approach is widely used in digital signal processing for predicting the signal power as a benefit because of its remarkable simplicity, and it has also been invoked for implementing temporal-domain spectrum prediction [15], [93], [128]-[130]. Other applications include speech- and audiocompression [131] as well as video compression [132].The most commonly used linear prediction models include the autoregressive (AR) model, the moving average (MA) model, the autoregressive moving average (ARMA) model and the autoregressive integrated moving average (ARIMA) model.

a) AR Model: With reference to the left column of the middle branch in Fig. 4, the AR model of order $m, A R(m)$, can be formulated as [133],

$$
X(t)=\sum_{i=1}^{m} \alpha_{j} X(t-i)+e(t) .
$$

In the spectrum prediction context, $X(t-i)$ represents the past observation before the $t$-th slot, while $X(t)$ and $e(t)$ represent the observation and error terms of the $t$-th slot. The expression $\sum_{i=1}^{m} \alpha_{j} X(t-i)$ aims for weighting the historical observations, where $m$ is the length of the prediction memory window and $\alpha_{j}$ is the weighting parameter of the AR model. Reference [15] used a second-order autoregressive model, i.e. $\operatorname{AR}(2)$, for performing spectrum prediction in the time domain, where the coefficient $\alpha_{j}$ has been obtained as the solution of the Yule-Walker equations. Then a Kalman filter has been used for predicting the state of a spectrum hole. In [130], the channel occupancy status is converted into a binary form and the AR model is used for spectrum occupancy characterization as well as for prediction. Finally, artificial signals complying with the Global System of Mobile
(GSM) communication standard were generated for testing the performance of prediction. Furthermore, AR models have also been applied for predicting both the channel state transitions in fading channels [134], [135] and the channel occupancy [93].

b) MA Model: The MA model is similar to the autoregressive model, except that the predicted value for the observation in the $t$-th slot depends on the error values observed in the past [136], rather than on the current values.

The MA model of order $n$, namely $M A(n)$, can be formulated as [133],

$$
X(t)=\sum_{i=0}^{n} \beta_{i} e(t-i)
$$

where, the notations $X(t)$ and $e(t)$ are the same as in the AR model, while $\beta_{j}$ is the parameter of the MA model and $\beta_{0}=1$.

In [137], when analysing the Chinese TV-band channels spanning from $603.25 \mathrm{MHz}$ to $843.25 \mathrm{MHz}$, the results indicate that the MA model is more suitable for predicting the strength of television signals than the 'experience based' method. Additionally, in [129], a non-linear exponential moving average (EMA) model is proposed, whose weighting factor decreases exponentially for each older data point, in order to put more emphasis on the recent observations, while giving some cognizance to former observations.

c) ARMA Model: Still referring to the left column of the middle branch in Fig. 4, the MA model is combined with the AR model to form an autoregressive moving average model $A R M A(m, n)[16]$ as follows:

$$
X(t)+\sum_{i=1}^{m} \alpha_{j} X(t-i)=\sum_{i=0}^{n} \beta_{i} e(t-i),
$$

where the notations of $X(t)$ and $e(t)$ are the same as in the AR and MA models. Furthermore, $\alpha_{j}$ and $\beta_{j}$ are the parameters 


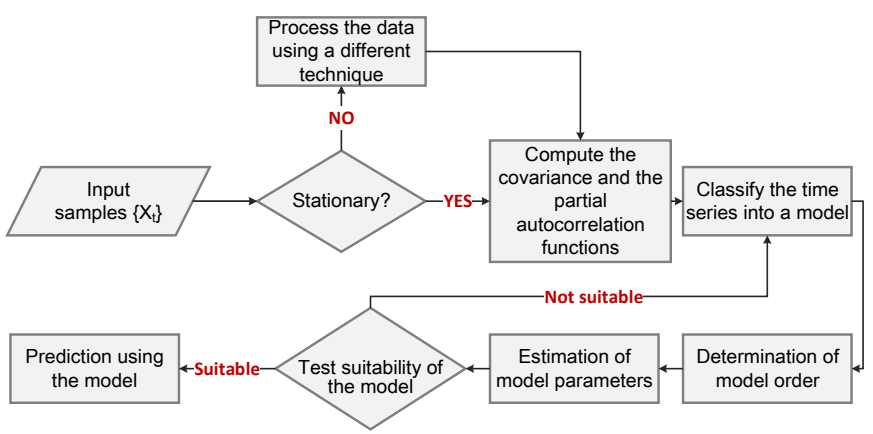

Fig. 5: Linear prediction (LP) structure.

of the ARMA model, $\beta_{0}=1$. In [138], the ARMA model shows a better prediction performance in channels which have cyclic behaviors compared to the performance in forecasting the Bluetooth and $\mathrm{WiFi}$ channels that have more random behaviors.

d) ARIMA Modeling: The most important feature of the more flexible ARIMA model is that it is capable of handling non-stationary time series as well as rapidly decaying autocorrelation functions [139], etc. The general $A R I M A(m, n, d)$ model is formulated as [136],

$$
W(t)=\sum_{i=1}^{m} \alpha_{j} W(t-i)+\sum_{i=0}^{n} \beta_{i} e(t-i),
$$

where we have $W(t)=X(t)-X(t-d)$, while $\alpha_{j}$ and $\beta_{i}$ are the parameters of the ARMA model of order $m, e(t)$ represents random errors, and $d$ is the degree of non-stationary homogeneity.

The overall process of using these models is illustrated in Fig. 5 adopted from [128]. The samples $X$ of the time series constitute the input of this procedure. As seen in Fig. 5, we first judge the degree of stationarity for the input data, and if the samples are not deemed to be stationary, they will have to be processed by different techniques. Following this step, we calculate both the covariance function as well as the partial autocorrelation function, and then classify $X$ into a specific model. Finally, we compute $m$ and $n$. As seen in Fig. 5, we also have to estimate the parameters $\alpha_{j}$ and $\beta_{i}$. Finally, we use the selected model to forecast the prospective values.

2) Markov Model (MM) Based Methods: Let us now consider the second column of the middle branch in Fig. 4. The Markov chain based modelling of sub-band PUs was validated by analyzing real-world measurements in the paging spectrum band in [140]. The Markov model has an appealingly simple structure characterized by its state-transition matrix. In this subsection, we will discuss a pair of temporal prediction methods, namely the Hidden Markov models (HMM) and the partially observable Markov decision processes (POMDP), also featuring in Fig. 4.

a) HMM: According to [141], an HMM is usually formulated as $\lambda=(\boldsymbol{\pi}, \boldsymbol{A}, \boldsymbol{B})$, where $\boldsymbol{\pi}$ represents the initial state probability vector, defined as:

$$
\boldsymbol{\pi}=\left[\boldsymbol{\pi}_{i}\right]=P\left(q_{t}=s_{i}\right), 1 \leq i \leq N,
$$

where $S=\left\{s_{1}, s_{2}, s_{N}\right\}$ denotes $N$ different states in a Markov chain and $q_{t} \in S$ represents the state at time instant

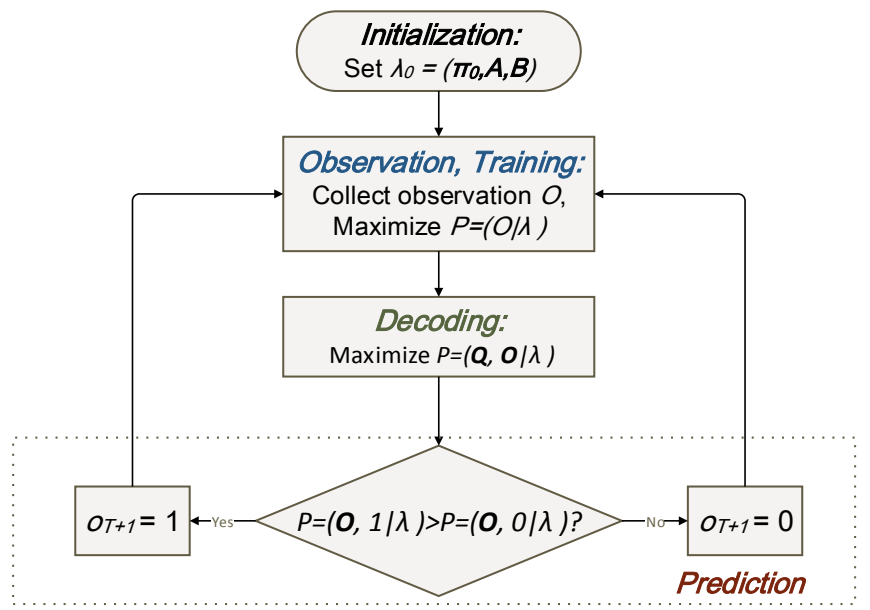

Fig. 6: HMM prediction process.

$t$, where $\pi_{i}$ satisfies the conditions $0 \leq \pi_{i} \leq 1, \sum_{i=1}^{N} \pi_{i}=1$. Furthermore, $\boldsymbol{A}=\left[a_{i j}\right]$ is the state transition matrix, which defines the probability of traversing from one state to another, formulated as:

$$
a_{i j}=P\left(q_{t}=s_{j} \mid q_{t-1}=s_{i}\right), 1 \leq i, j \leq N .
$$

Furthermore, $\boldsymbol{B}=\left[b_{i k}\right]$ is the observation probability matrix that offers the option of producing different observed values, while being in a particular state, which is encapsulated in:

$$
b_{j k}=P\left(o_{t}=v_{k} \mid q_{t}=s_{j}\right), 1 \leq j \leq N, 1 \leq k \leq M,
$$

where $\boldsymbol{V}=\left\{v_{1}, v_{2}, \ldots, v_{M}\right\}$ represents the space containing $M$ observed symbols and $o_{t}$ is the value observed at time instant $t, o_{t} \in V$. Note that we have $0 \leq a_{i j}, b_{j k} \leq 1, \sum_{j=1}^{N} a_{i j}=1$ and $\sum_{k=1}^{M} a_{j k}=1$.

Traditionally, a standard HMM predictor is based on the discrete-time model introduced in Section II, where the time is slotted and the state space is binary, with the spectrum slot being either in state $s_{i}=1$ or $s_{i}=0$. The set $\boldsymbol{O}=\left\{o_{1}, o_{2}, \ldots, o_{T}\right\}$ may be used for representing the past observation sequence in $T$ consecutive slots. In order to predict the state in the $(T+1)$-st slot, a HMM predictor of the standard form may be designed by obeying the following four steps [20], [111], which are illustrated in Fig. 6 and detailed as follows:

Step 1: Initialization. Set the initial parameters of the HMM $\lambda_{0}=\left(\boldsymbol{\pi}_{0}, \boldsymbol{A}_{0}, \boldsymbol{B}_{0}\right)$.

Step 2: Observation. The CR senses the sub-band spectrum and collects the observed data $\boldsymbol{O}=\left\{o_{1}, o_{2}, \ldots, o_{T}\right\}$.

Step 3: Training. Given the observed sequence, the parameters of the HMM will be adapted by invoking an appropriate training approach, such as the Monte-Carlo method, and the Baum-Welch algorithm [141], for maximizing the likelihood associated with the model and defined as $P(\boldsymbol{O} \mid \lambda)$.

Step 4: Prediction. The state observed at slot $(T+1), o_{T+1}$, can be predicted by the following rule:

$$
o_{T+1}= \begin{cases}1, & \text { if } P(\boldsymbol{O}, 1 \mid \lambda) \geq P(\boldsymbol{O}, 0 \mid \lambda), \\ 0, & \text { if } P(\boldsymbol{O}, 1 \mid \lambda)<P(\boldsymbol{O}, 0 \mid \lambda) .\end{cases}
$$


This standard HMM approach has been investigated in [14], [20], [95], [111], [140], [142], [143] for the sake of predicting the future states and the idle/busy durations of the channel. In [95], the HMM is used for predicting the spectrum usage patterns, which are assumed to be deterministic Poisson distributed. Given a sequence of predicted states, the CR will dynamically choose these surmised frequencies for its use, even though the spectrum occupancy should generally be modeled by stochastic distributions, as previously mentioned in Section II. This algorithm was also advocated in [142], matching its output to the data collected from the 450-470 $\mathrm{MHz}$ band in Australia, but the specific implementation of the algorithm was not detailed in [142]. Meanwhile, the authors of [14] considered a realistic propagation environment that took into account the time delay incurred both by the hardware and software. It should be mentioned that in [14] the parameters of the HMM used are obtained statistically, without employing any training for the model. Instead, the authors verify the proposed method using measured WiFi data instead of artificial data. Both the model complexity and the computational complexity have been studied in order to provide a qualitative characterization of the HMM performance, which were complemented by a range of implementation issues arising during the design [118]. As the number of states $N$ increases, the number of model parameters to be estimated also increases. The computational complexity of the HMM based predictor is directly related both to the number of states $N$ and to the size of the observation sequence $T$.

Moreover, MM or HMM-based methods work well under the assumption of having memoryless or Markovian spectrum state evolution, where the future state depends only on the relevant information about the current, not on information from distant the past. The Markovian property has been validated by analyzing real-world measurements in the paging spectrum band in [140], which motivates the research of HMM-based spectrum inference methods. Most of the related studies have focused on first-order and stationary HMM. However, in practical systems, the spectrum state of the future may depend on a relatively long historical information (see the tide effects in the GSM bands [209]). The state distribution of PUs may also dynamically change. In order to improve the performance of the standard HMM, in recent years researchers have developed several sophisticated HMMs by carefully considering the stationarity and the high-order Markovian nature of the spectral slot occupancy pattern. Some representative advances are summarized below as follows:

- Non-stationary Hidden Markov model (NS-HMM). Here we are still considering the Markov-modeling belonging to the second branch of the temporal algorithms seen in Fig. 4. As mentioned above, the PU's spectral slot occupancy pattern is assumed to be deterministically distributed, when the standard HMM model is used. However, in reality the PU's behaviors obey time-varying non-stationary DTMC [89]. Under these circumstance, conventional HMMs may fail to adequately characterize the PU's dwelling time distributions [17]. For this reason, the NS-HMM was employed for modeling the channel's status in [17] in order to characterize the idle/busy duration of the PU's channel. The NS-HMM can be described as $\lambda_{N S}=[\boldsymbol{\pi}, \boldsymbol{A}(t), \boldsymbol{B}]$, which replaces the static transition probabilities of the conventional HMMs with dynamic ones. In [17], the parameters of the NSHMM are inferred through Bayesian inference with the aid of Gibbs sampling, and the impact of different model parameters on the model's accuracy has been investigated. It was found that as expected the channel quality experienced by the $\mathrm{CR}$ is an increasing function of the sensing accuracy and of the estimated idle duration. In [118], the so-called expectation maximization based algorithm was developed for calculating the parameters of a NSHMM. Numerical experiments relying on real spectrum measurement data have been carried out for demonstrating that the NS-HMM outperforms the traditional HMMbased approaches.

- Hidden Bivariate Markov Model (HBMM). As an extension of the standard HMM, HBMMs have been proposed for more accurately characterizing the transmission pattern of a PU [144]. In contrast to the standard univariate HMM, the HBMM incorporates a number of additional variables by introducing a state duration distribution for modeling the channel usage in $\mathrm{CR}$, rather than using the geometric duration distribution of a standard HMM. In [144], the HBMM was described in form of the function $\lambda_{B}=(\boldsymbol{\pi}, \boldsymbol{G}, \boldsymbol{\mu}, \boldsymbol{R})$, where $\boldsymbol{\pi}$ is the initial state probability vector reminiscent of that in the standard HMMs, $\boldsymbol{G}$ is the state transition matrix, while $\boldsymbol{\mu}$ and $\boldsymbol{R}$ represent the vector of observed receive signal strength average and variance. Repeatedly using the definitions of the standard HMM parameters, let $\boldsymbol{Z}=\boldsymbol{O} \times \boldsymbol{S}=\left[Z_{t}\right]=$ $\left(o_{t}, q_{t}\right)$ denote the specific value returned by the bivariate Markov chain. The state transition matrix can be defined as:

$$
\boldsymbol{G}=\left[g_{a b}(i j)\right]=\left[P\left(Z_{t+1}=(b, j) \mid Z_{t}=(a, i)\right)\right] .
$$

The parameter $\lambda_{B}$ may be estimated by extending the Baum algorithm [145], which is simpler than an explicit duration estimating algorithm. When determining the model parameters, the authors of [144] apply forwardbackward recursions for predicting the spectral slot state at a future time instant. The performance of the HBMM in spectrum sensing and prediction was characterized by numerical results in [144], demonstrating that the HBMM is capable of more accurate state predictions than a standard HMM.

- Higher-order HMM. Still referring to Fig. 4, we note that although the first-order standard HMM has been broadly adopted for predicting the channel's state, this model does not make full use of the historical information, since a state only depends on the immediate preceding state. To alleviate this problem, a higher-order HMM was also proposed for predicting the next channel state [146]. A higher-order HMM can be formulated as $\lambda_{H O}=\left(\boldsymbol{\pi}, \boldsymbol{A}_{H O}, \boldsymbol{B}\right)$. In contrast to the standard HMMs, $\boldsymbol{A}_{H O}=\left[a_{i j}^{H O}\right]$ is the state transition matrix representing the transition from the states in the previous $D$ slots to 
the current state, which can be defined as [146]:

$$
\begin{aligned}
\boldsymbol{A}_{H O}= & {\left[a_{i j}^{H O}\right]=P\left(q_{t+1}=s_{i_{D+1}} \mid q_{t}=s_{i_{D}}, \ldots,\right.} \\
& \left.q_{t-D+1}=s_{i_{1}}\right), i_{1}, i_{2}, \ldots, i_{D}, i_{D+1}=1, \ldots, N .
\end{aligned}
$$

When considering the computational complexity versus model accuracy trade-offs, there are several variants of the higher-order HMMs [146]-[149]. By relying on realworld WiFi signals recorded, it was shown that the performance of the proposed approach is significantly better than that of the nearest neighbor prediction relying on first-order HMMs, especially when the order of the HMM increases [146].

b) POMDP: The last member of the MM-based temporal methods seen in Fig. 4 is constituted by the POMDP family, which is a generalization of a Markov decision process that tolerates uncertainty about the state of a Markov process and allows the acquisition of environmental information [150]. Based on DTMC model of Section II [89], a POMDP applied in CRNs is defined by the six-tuple $(\boldsymbol{S}, \boldsymbol{A}, \boldsymbol{T}, \boldsymbol{R}, \boldsymbol{Z}, \boldsymbol{O})$ [151][154], where

$S$ is the channel state space $\{0$ (idle), 1 (busy) $\}$;

$A$ is a discrete and finite set denoting the CR actions, i.e. as $\left\{a_{1}\right.$ (access),$a_{2}$ (no access) $\}$;

$\boldsymbol{T}: \boldsymbol{S} \times \boldsymbol{A} \rightarrow \boldsymbol{\Pi}(\boldsymbol{S})$ is the state-transition function. If $s$ is the current channel state and action $a$ is chosen by the decision maker, the process will move to a new state $s$ with the probability of $\boldsymbol{T}\left(s^{\prime} \mid s, a\right)$.

$\boldsymbol{R}: S \times \boldsymbol{A} \rightarrow \mathbb{R}$ is the reward function that defines the expected immediate reward $\boldsymbol{R}(s, a)$ received, when the process is in state $s$ and action $a$ is taken;

$Z$ is a finite set of observations the CR infers from the radio environment;

$\boldsymbol{O}: \boldsymbol{S} \times \boldsymbol{A} \rightarrow \boldsymbol{\Pi}(\boldsymbol{Z})$ is the observation function and for each action and resultant state, $O\left(s^{\prime} \mid a, o\right)$ denotes the probability of observing $o$ and moving to state $s^{\prime}$ after taking action $a$;

The POMDP based CR engine [155] is depicted in the Fig. 7, with the state predictor being the core component in the process [151], [153]. In this procedure, $b$ is the 'belief state', which describes the probability that the channel's state is idle, summarizing all the past information necessary for formulating the allocation decision $\pi$ [154]. The channel state predictor computes the probability $b$ based on a combination of the most recent value, on the current observation and on the previous action. More specifically, the authors of [153] proposed an approach for channel state prediction based on POMDP by finding the optimal policy that maximizes some aspect of the reward. In this approach, the probability $b^{\prime}\left(s^{\prime}\right)$ that the future state $s^{\prime}$ is idle can be calculated by the Bayesian formula as follows

$$
b^{\prime}\left(s^{\prime}\right)=P\left(s^{\prime} \mid a, b, o\right)=\frac{\sum_{S} O\left(o_{t+1} \mid s^{\prime}, a\right) T\left(s^{\prime} \mid s, a\right) b(s)}{P\left(o_{t+1} \mid b, a\right)} .
$$

The numerical results provided in [152], [156] have shown that the performance of the spectrum access approach using the POMDP based CR improves over time upon gleaning increasingly more accurate information concerning the channel's state inferred from the accumulated observations. As a benefit,

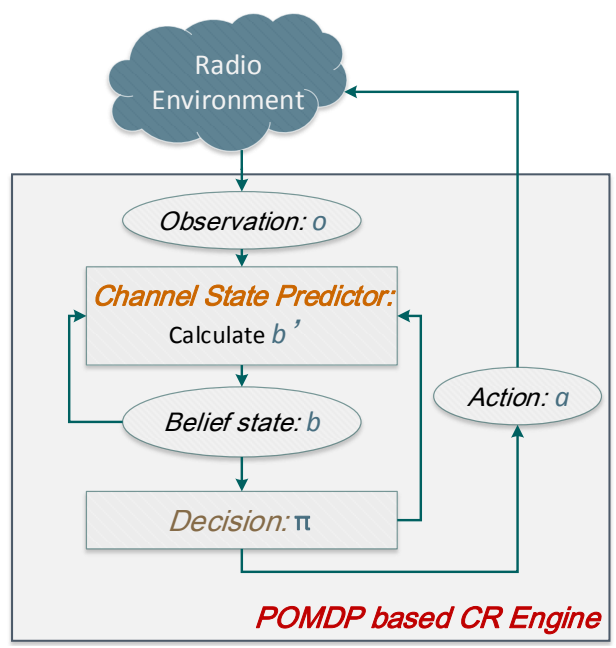

Fig. 7: POMDP based prediction.

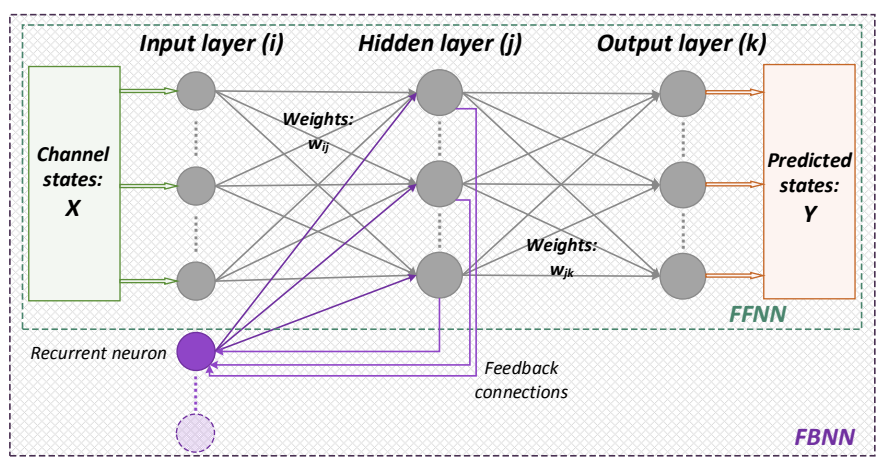

Fig. 8: Artificial neural network (ANN) based prediction.

the collisions of CRs with PUs were substantially reduced. However, a problem concerning the POMDP based approach is its potentially excessive complexity, especially when the number of channels is high. To tackle this problem, more research is required for developing efficient algorithms for solving POMDP problems.

3) Artificial Neural Network (ANN) Based Methods: Let us now move on to the third family of the temporal methods portrayed in Fig. 4. As a key technique in machine learning, the ANNs have been extensively applied to communications, signal processing, intelligent control, etc. They represent a class of flexible nonlinear models based on parallel computation, which consist of both input and output layers as well as intermediate layers composed of artificial neurons [157]. In CRNs, an ANN allows for learning the PU's behavioral patterns by invoking a training set of spectrum data, learn these patterns, and then capitalize on it for classifying new patterns and for making forecasts. Because of its high prediction accuracy and capability of learning, ANNs constitute a popular approach of predicting the spectrum state's evolution. Generally, depending on their architecture, we can categorize ANNs into two classes: feedforward and feedback neural networks [158]. The architecture of ANNs invoked for predicting channel states is depicted in Fig. 8.

a) Feedforward Neural Networks (FFNNs): Feedforward neural networks enter the spectrum data strictly into the input 
layer and give the states predicted by the output layer without any feedback. Referring to the rich literature concerning FFNN based algorithms invoked for spectrum prediction [20], [157], [159]-[168], we will first provide a brief overview of typical multilayer feedforward neural networks (MFNNs), also known as the multilayer perceptron (MLP). Then we will describe the overall spectrum prediction procedure of neural networks.

Based on the PU's activity modeled by DTMC, where the input and output data are represented by binary sequences, MFNNs rely on neurons as their basic computing unit for calculating a weighted sum of the inputs and perform a nonlinear transformation of the sum [20], [160], [168], which can be expressed as follows [20], [168]:

$$
\begin{aligned}
y_{j}^{l} & =f\left(a_{j}^{l}\right), \\
a_{j}^{l} & =\sum_{i} y_{i}^{l-1} w_{j i}^{l},
\end{aligned}
$$

where $y_{j}^{l}$ is the output of a neuron $j$ in the $l_{t h}$ layer; $w_{j i}^{l}$ represents the adaptive weights connecting the neuron $j$ in the $l_{t h}$ layer and neuron $i$ in the $(l-1)_{s t}$ layer; $a_{j}^{l}$ is the weighted sum of the inputs yielding the outputs of the former layer, and $f\left(a_{j}^{l}\right)$ is the activation function, often represented by the tangent sigmoid function [160]:

$$
y_{j}^{l}=\frac{2}{1+e^{-2 a_{j}^{l}}}-1 .
$$

Before performing spectrum prediction, there should be a training process for MFNNs. First, the observed spectrum data vectors $\boldsymbol{X}(t-\tau+1, t)=\left\{x_{t}, x_{t-1}, \ldots, x_{t-\tau+2}, x_{t-\tau+1}\right\}$ are imported into the input layer and the estimated output data $y_{t+1}^{o}$ is calculated using the activation function. Then, the difference between the desired value $x_{t+1}$ and its estimation $y_{t+1}^{o}$ is denoted as the error $e_{t}$, which can be expressed as follows [20], [160]:

$$
e_{t}=x_{t+1}-y_{t+1}^{o} .
$$

Finally, in order to minimize the prediction error $e_{t}$, the adaptive weight $w_{j i}^{l}$ will be updated by repeatedly using the back propagation algorithm [169] until the prediction error reaches its minimum. In [160], the prediction error is measured by the mean squared error (MSE), denoted as $E(w)$, which can be computed as follows [160]:

$$
E(w)=\frac{1}{2} \sum_{t} e_{t}^{2}=\frac{1}{2} \sum_{t}\left(x_{t+1}-y_{t+1}^{o}\right)^{2} .
$$

Once the training process is completed, the future spectrum state can be predicted by capitalizing on the observations with the aid of the MFNNs. In [20], the predicted value $y_{t+1}^{o}$ is decided by invoking a threshold at the output layer, which can be expressed as follows:

$$
y_{t+1}^{o}= \begin{cases}0, & \text { if } y_{t+1}^{o} \geq 0 \\ 1, & \text { if } y_{t+1}^{o} \leq 1\end{cases}
$$

It is noteworthy that the learning speed of the traditional FFNNs, like MFNNs, is generally slower than desired, which would naturally evolve the attainable prediction efficiency. This problem was solved by the extreme learning machine
(ELM) of [177]. Furthermore, the authors of [159] optimized the original ELM, while in [158] the optimally pruned extreme learning machine (OP-ELM) was used for spectrum prediction. OP-ELM based spectrum prediction also relies on the structure of single hidden layer neural networks (SLFNs), just like the original ELM, where the input weights are randomly assigned, but the output weights are calculated using a different procedure. According to the experiments conducted in [158], [159], the OP-ELM algorithm is more robust and flexible than the original ELM, showing a higher learning speed and a higher accuracy compared to other FFNNs.

b) Feedback Neural Networks (FBNNs): The only difference between FBNNs and FFNNs is the presence or absence of feedback links that span from the outputs of neurons to the inputs of neurons in the previous layer [178], [179], as depicted in Fig. 8. The FBNN requires the data to be passed both forward as well as backward, hence imposing a high complexity and often making the FBNNs 'confused' or unstable. In this sense, the forecasting performance of FBNNs may become less attractive than that of FFNNs [180]. FBNNs gleam information from the sequence or time dependence of the inputs and the inputs themselves, which means that the features detected in previous patterns form a part of each new pattern.

Therefore, when we want to take the previous spectrum observations into consideration in order to sense or predict the spectrum, the FFNNs discussed above are less pragmatic for us. In [178], Elman recurrent neural networks (ERNN) is used for radio frequency (RF) multivariate time series modelling in order to predict the spectral evolution. This feedback method leads to more intelligent $\mathrm{CR}$ decisions for exploiting the expected spectrum opportunities, thereby leading to optimized spectrum usage and interference avoidance [178]. Although ERNN is powerful in predicting the time domain sequences, it has slow convergence and does not excel in terms of determining the network structure [181]. The Echo State Network (ESN) concept is proposed as a new technique of overcoming these problems in [182]. Taking this as a basis, the authors of [183] further proposed a method based on an improved form of ESN for spectrum prediction. The proposed ESN structure is also visualized in [183]. In contrast to the ERNN, the intermediate hidden layers are replaced by a cycle reservoir associated with fixed feedback connections and randomly connected neurons [183]. The parameters of the improved ESN are calculated using particle swarm optimization (PSO). It should be noted that the PSO is known to outperform the traditional back propagation algorithms in training ANNs, as a benefit of their faster convergence and lower complexity [165], [184]. Experiments have been conducted in [183] to demonstrate that the performance of the optimal ESN is better than that of the ERNN in predicting both the specific state and the state duration of the primary spectrum, since it reduces the training time required for obtaining a higher prediction accuracy.

4) Pattern Mining (PM) Based Methods: PM is a classic data mining technique conceived for discovering underlying patterns in large amounts of data. In CRNs, the patterns usually reflect the rules hidden in the spectrum occupancy of a channel. The motivation of finding the rules arises from the 
desire to analyze historical spectrum data for predicting the future channel states, so as to improve the attainable spectral efficiency and hence to reduce the collision rate. In previous research, the spectrum usage prediction has relied on frequent pattern mining (FPM) [119] and partial periodic pattern mining (PPPM) [16]. Both methods are based on a binary channel state model.

- FPM. In the context of CRNs, the channel state values observed are delivered in a sequence and serve as the spectrum occupancy history database. The mission of FPM is to find the specific sequences that appear in the data set with a frequency above a threshold, which are referred to as a frequent sequential patterns [185]. Thus, it becomes possible to predict the next state or next sequence. Although the FPM of [119] is two-dimensional, its ability to predict the future spectral states is only known in the time domain.

- PPPM. In contrast to FPM, the partial periodic patterns observe the PU's features during certain time periods instead of the entire time span [186]. Since the propagation environment is stochastic and the PU's behaviors may not show an obvious regularity. The patterns only become relevant for certain time periods of the day [16]. The procedure of PPPM based prediction can be described as follows [16]:

Step 1: Process the binary channel state values and generate candidate patterns;

Step 2: Count the support of each candidate pattern;

Step 3: Eliminate the redundant candidate patterns;

Step 4: Generate the prediction rules and calculate the probability of the future channel states.

Additionally, the algorithm based on PPPM was also used for predicting the channel state duration in [16] so as to reduce the collision probability owing to the PU's short absence.

5) Other Algorithms: Other relevant temporal methods found in the literature include Bayesian inference (BIF), learning automata (LA) and support vector machines (SVM). BIF is known as a classic method of solving the state prediction problem in CRNs as a critical part of the Markov systems, like the aforementioned NS-HMM and POMDP. It provides a general unifying framework for sequential state estimation. The procedure of BIF based prediction continuously updates the a posteriori distribution in a recurrent manner, based on the influx of spectrum data. Assuming that the evolution of the state is Markovian, BIF can make predictions based both on the observations and on the a posteriori distribution. BIFbased prediction is proposed in [17] [187] for estimating the distribution of idle durations in the PU's traffic. The algorithm proposed in [17] combines BIF with NS-HMM to predict the channel quality, which is capable of improving the efficiency of dynamic spectrum access. Compared to the traditional maximum likelihood technique, the BIF technique is capable of performing better, when processing a limited number of samples, and the channel availability may be reliably predicted after estimating the prevalent spectrum usage patterns [188]. In [157], a LA technique is utilized for predicting the spectrum opportunities in CRNs. The LA technique first generates the PU's activity model according to the characteristics of the PU's behavior. Then, the training and testing stage updates the parameters of the models, and finally the performance of the model is assessed in order to correct the prediction of the system's behavior. In comparison to the MFNNs, the LA has shown a high performance, despite its simple structure [157].

The SVM technique has been widely used to make time series predictions in many scientific fields, such as financial marketing, power supply and medical sciences [189] as well as for spectrum prediction in CRNs. The application of SVM to solve regression problems is termed as support vector regression (SVR). In [190], SVR is used for predicting the probability density of the idle/busy CR state duration. In [191], SVR is applied for processing the spectrum occupancy data.

\section{B. Spectrum Inference in Frequency Domain}

Section III-A has reviewed some of the popular techniques conceived for predicting the future spectrum occupancy in the time domain. Further studies are well dedicated to inferring the states of other channels in the light of already acquired sensing or predicted results [93], [119], [130], [192], [193]. The spectral occupancy correlation between adjacent channels has been evaluated by experiments in [194]-[196]. This correlation serves as the most important information representing the relationship of different channels within the same services. In other words, the more correlated two channels are, the more accurate predictions will be made. The authors of [192] exploited the spectral correlations for inferring the availabilities of other channels in order to improve the throughput. The new concept of channel availability vector was introduced for characterizing the spectral occupancy information.

In [193], predicting the states of unsensed channels is formulated as a matrix completion problem. The classic technique of belief propagation (BP) [197] is applied to fill the matrix with predicted states. It is found that the BP scheme is more suitable for specific matrix types, where the adjacent elements are highly correlated [29]. In [198], Bayesian networks are proposed for jointly modeling the spectral-domain and spatialdomain correlations, where the authors introduced statistical inference for evaluating the spectrum occupancy. The correlation across the frequency dimension was exploited to enhance the estimation of spectrum occupancy in wideband spectrum sensing [195], [196].

Yin et al. [119] has proposed a frequency pattern matching algorithm operating in two-dimensions (FPM-2D) for spectrum inference, which searches through all relevant 2D patterns [119]. Once these patterns have been obtained, we can compute the probabilities of future channel states and estimate the channel's availability. Their algorithm outperforms the first-order HMM-based predictor in terms of its prediction accuracy. Similarly, frequency domain correlation techniques were also introduced in [93] by modeling the neighboring channels in pairs. Simultaneously considering multiple frequency bands, the classic AR models which do not need a priori knowledge on the communication environment were also employed to reduce the complexity [93]. This algorithm performs well for deterministic usage patterns. 
The investigations of [93], [119], [192], [196] clearly quantify the performance improvements achieved by spectral correlation based inference.

\section{Spectrum Inference in the Geographic Spatial Domain}

From a spatial-domain perspective, there are two directions for spectrum inference:

- One of them aims to infer the spectrum state information, while taking both the positions and movements of CRs into consideration;

- The other one infers geographical information, including the primary system's coverage contours, service areas and so on.

1) The first direction: In the first direction, both the $\mathrm{BP}$ technique and the Bayesian networks discussed in the frequency domain are also capable of taking the spatial correlations into account for inferring the channel states from those of other locations.

In the static spectrum environment, the interference powers experienced at different $\mathrm{CR}$ nodes are inferred by exploiting the spatial variation of interference. Specifically, both semisupervised dictionary learning [200] and compressive sensing are employed for the interpolation of unobserved interference in the spatial domain, incorporating the CR network topology.

Since the movement of CRs directly affects the spectrum availability in a specific geographic area, spectrum prediction in the time-space domain constitutes and important research area. The traditional static model is extended to a dynamic mobililty model in [201]. The CRs' mobility and the PUs' activity can be jointly considered to infer both the spectrum occupancy and the interference constraints for a certain period under different relative positions between the PUs and CRs. Based on this scheme, both a greedy and a fair prediction algorithms were proposed in [201] to make the spectrum exploitation more effective, hence maintaining fairness in spectrum allocation. A SVM is used for inferring the spectrum occupancy evolution considering both the timedomain and geographic distribution characteristics [202]. A joint feature-vector extraction method is designed by analyzing both the CRs' movement and the PUs' behavior. Again, a SVM based inference mechanism is introduced for expediting the convergence speed, which is shown to have a better inference performance than the algorithms solely depending on the speed and location information, as in [202]. It was found that carefully choosing the parameters is capable of mitigating the performance loss caused by high-speed CRs having erratic movements. In [203], the SVM was invoked both for predicting the handoff point and the idle channels with a high precision. In addition to the spectrum availability, the link availability between the CRs was also inferred by the authors of [204]. By invoking this novel approach, a more reliable path can be found for dynamic routing in CRNs.

2) The second direction: For the TV services, the primary receivers must be protected from harmful interference [18]. This approach makes full use of the effective antenna height, effective isotropic radiated power and terrain information to map the primary users' coverage contour through Fresnel diffraction theory. The Google Earth software plays an indispensable role in TV white space prediction. Based on the primary users' coverage contour inference, the potential interference and the collisions imposed by the CRs may be significantly reduced. In [205], a location predictor is proposed, where the historical changes of the PU's geographic locations are represented by a directed graph having weighted edges. Once a spectrum occupancy prediction is requested, all the edges originating from the starting point are listed and then the destination is predicted according to the calculated maximum weights.

\section{Spectrum Inference in Joint Multiple Domains}

In addition to spectrum inference techniques in single domain (i.e., time, frequency and space) mentioned above, there are also recent studies on joint multiple-domain spectrum inference. Specifically, the work in [119] is one of the first studies to develop joint time-frequency spectrum prediction algorithm, where two-dimensional frequent pattern mining is proposed to analyze binary historical spectrum occupancy data. In [210], the authors develop an algorithm for spectral-temporal two-dimensional spectrum prediction with incomplete historical data by exploiting the approximate lowrank property of real-world spectrum measurement matrices. In [211], a robust spectral-temporal two-dimensional spectrum prediction algorithm is proposed under an assumption on the sparsity of abnormal or corrupted historical data. In [212], a matrix completion-based algorithm is developed for TV white space database construction via joint spectrum sensing in time domain and spectrum inference in spatial domain. In [213], the authors propose the concept of spectrum tensor and develop a multi-dimensional (including, time, frequency, and space) spectrum inference algorithm for spectrum map construction by invoking the recent advances in tensor completion. Moreover, the recent tutorial papers [214], [215] propose to combine spectrum sensing and spectrum inference into spectrum database for providing spectrum services for users, and also highlight that joint multiple-domain spectrum inference is an active research trend.

\section{E. Summary and Insights}

As discussed above, there are a wealth of contributions on spectrum inference. In this subsection, we offer a brief summary and discussions of the existing spectrum inference algorithms. First of all, the major developments of spectrum inference/prediction techniques are summarized at a glance in Tables II and III in the chronological order. Then, the advantages and technical challenges of various spectrum inference algorithms are summarized in Table IV, according to a priori information concerning the processed data and to the resultant complexity and accuracy constraints.

Furthermore, there are also several major insights based on the comprehensive survey of the existing studies detailed as follows. Firstly, while the majority of the existing studies focused on spectrum inference in the time domain, in the past decade, spectrum inference in joint multiple domains has been gaining increasing research interests. For instance, the 
TABLE II: Major developments of spectrum inference techniques (Part 1) (T/E: Theoretic/Experimental work; U/O/I: Underlay/Overlay/Interweave).

\begin{tabular}{|c|c|c|c|c|c|c|}
\hline Year & Dimension & Algorithm & Reference & Key Contribution(s) & T/E & $\mathrm{U} / \mathbf{O} / \mathbf{I}$ \\
\hline \multirow{3}{*}{2007} & \multirow[b]{3}{*}{ Time } & HMM & {$[95]$} & $\begin{array}{l}\text { proposed to use the HMM based prediction algorithm in comparison with } \\
\text { traditional CSMA based algorithms. }\end{array}$ & $\mathrm{T}$ & I \\
\hline & & AR & [130] & $\begin{array}{l}\text { proposed an AR algorithm with different orders in predicting spectrum occu- } \\
\text { pancy status. }\end{array}$ & $\mathrm{T}$ & I \\
\hline & & AR & [135] & $\begin{array}{l}\text { proposed an AR algorithm to predict channel state transitions over fading } \\
\text { channels. }\end{array}$ & $\mathrm{T}$ & I \\
\hline \multirow{5}{*}{2008} & \multirow{5}{*}{ Time } & FFNNs & [168] & $\begin{array}{l}\text { proposed a spectrum prediction algorithm using multilayered feedforward neural } \\
\text { networks for better PHY rate adaption. }\end{array}$ & $\mathrm{T}$ & I \\
\hline & & SVR & [191] & $\begin{array}{l}\text { proposed SVR-based prediction for nonlinear, non-stationary and complex data } \\
\text { series. }\end{array}$ & $\mathrm{T}$ & I \\
\hline & & ARIMA & [206] & $\begin{array}{l}\text { proposed a seasonal ARIMA algorithm to analyse the spectrum occupancy and } \\
\text { make forecasts. }\end{array}$ & $\mathrm{T}$ & I \\
\hline & & AR & [15] & $\begin{array}{l}\text { applied the AR model presented in [135] and a Kalman filter to predict spectrum } \\
\text { holes. }\end{array}$ & $\mathrm{T}$ & I \\
\hline & & MA & [207] & $\begin{array}{l}\text { proposed an exponential weighted moving average based approach to predict } \\
\text { spectrum occupancy. }\end{array}$ & $\mathrm{T}$ & I \\
\hline \multirow{6}{*}{2009} & \multirow{6}{*}{ Time } & FFNNs & [167] & adopted the approach of [168] for channel selection. & $\mathrm{T}$ & I \\
\hline & & HMM & [140] & validated the Markov chain-based modeling of the spctrum usage. & $\mathrm{E}$ & I \\
\hline & & POMDP & [154] & proposed a POMDP framework for DSA considering the energy constraint. & $\mathrm{T}$ & I \\
\hline & & BIF & [208] & $\begin{array}{l}\text { provided a fast Bayesian statistical approximation method to infer the radio } \\
\text { signal's power. }\end{array}$ & $\mathrm{T}$ & $\mathrm{U} / \mathrm{O}$ \\
\hline & & MA & [128] & $\begin{array}{l}\text { discovered that the usage pattern of all Chinese TV band channels can be } \\
\text { modeled by the MA modeling method. }\end{array}$ & $\mathrm{T}$ & I \\
\hline & & MA & [129] & $\begin{array}{l}\text { presented the EMA based prediction approach to improve energy detection in } \\
\text { order to reduce the sensing time. }\end{array}$ & $\mathrm{T}$ & $\mathrm{I}$ \\
\hline \multirow{6}{*}{2010} & \multirow{4}{*}{ Time } & BIF & [198] & invoked a Bayesian network to infer the spectrum occupancy. & $\mathrm{T}$ & I \\
\hline & & FFNNs & [163] & $\begin{array}{l}\text { designed a MLP to predict the channel status without requiring a priori } \\
\text { knowledge of the statistics of channel usage. }\end{array}$ & $\mathrm{T}$ & I \\
\hline & & HMM & [146] & $\begin{array}{l}\text { extended the standard HMM prediction method of [140] by considering the } \\
\text { latency between spectrum sensing and data transmission. }\end{array}$ & $\mathrm{T}$ & I \\
\hline & & BIF & [187] & proposed a BIF method to estimate the distribution of state duration. & $\mathrm{T}$ & I \\
\hline & Space & SNG & [205] & proposed the SNG algorithm for predicting the mobility of cognitive users. & $\mathrm{T}$ & $\mathrm{U} / \mathrm{O}$ \\
\hline & Frequency, Space & MC & [193] & $\begin{array}{l}\text { proposed an efficient framework of BP for matrix completion to achieve a } \\
\text { reduced error rate. }\end{array}$ & $\mathrm{T}$ & $\mathrm{U} / \mathrm{O}$ \\
\hline \multirow{5}{*}{2011} & Frequency, Time & AR & [93] & $\begin{array}{l}\text { took the frequency dependence into consideration to optimize the performance } \\
\text { of the AR model. }\end{array}$ & $\mathrm{T}$ & I \\
\hline & \multirow{4}{*}{ Time } & SVM & [203] & $\begin{array}{l}\text { introduced the SVM model to infer the handoff point to reduce the collision } \\
\text { rate. }\end{array}$ & $\mathrm{T}$ & I \\
\hline & & ERNN & [178] & $\begin{array}{l}\text { proposed an ERNN approach and modeled the features of the PUs' activity as } \\
\text { a multivariate chaotic series. }\end{array}$ & $\mathrm{T}$ & I \\
\hline & & FFNNs & [162] & applied the MLP to measured data. & $\mathrm{E}$ & $\mathrm{I}$ \\
\hline & & HMM & [14] & $\begin{array}{l}\text { modified the HMM approach for single-user prediction and considered the time } \\
\text { delay of hardware platforms. }\end{array}$ & $\mathrm{E}$ & I \\
\hline \multirow[t]{3}{*}{2012} & \multirow[t]{2}{*}{ Time } & PPPM & [216] & $\begin{array}{l}\text { introduced a PPPM algorithm to mine the underlying spectrum occupancy } \\
\text { patterns to make forecasts. }\end{array}$ & $\mathrm{T}$ & $\mathrm{I}$ \\
\hline & & ARMA & [217] & $\begin{array}{l}\text { proposed a multichannel ARMA prediction filter based on a particular lattice } \\
\text { filter structure. }\end{array}$ & $\mathrm{T}$ & $\mathrm{I}$ \\
\hline & Frequency, Time & FPM & [119] & $\begin{array}{l}\text { developed a two-dimensional FPM algorithm for exploiting the spectral corre- } \\
\text { lations. }\end{array}$ & E & I \\
\hline \multirow{5}{*}{2013} & Space & DL & [200] & $\begin{array}{l}\text { proposed a specific dictionary learning framework to predict the interference } \\
\text { power levels in various locations. }\end{array}$ & $\mathrm{T}$ & $\mathrm{U} / \mathrm{O}$ \\
\hline & \multirow{4}{*}{ Time } & FBNNs & [183] & $\begin{array}{l}\text { proposed a ESN method to predict the state duration with the aid of an improved } \\
\text { parameter selection algorithm. }\end{array}$ & $\mathrm{T}$ & I \\
\hline & & HMM & [111] & applied the HMM algorithm in the HF spectrum for activity prediction. & $\mathrm{E}$ & I \\
\hline & & HMM & [144] & $\begin{array}{l}\text { proposed a hidden bivariate Markov model (HBMM) prediction method which } \\
\text { allows a phase-type dwell time distribution. }\end{array}$ & $\mathrm{T}$ & I \\
\hline & & HMM & [17] & proposed a NS-HMM to predict the channel quality. & $\mathrm{T}$ & $\mathrm{U} / \mathrm{O}$ \\
\hline \multirow{4}{*}{2014} & Space & SVM & [202] & proposed a SVM based spectrum mobility prediction algorithm. & $\mathrm{T}$ & I \\
\hline & \multirow{2}{*}{ Time } & LA & [157] & proposed the LA technique to predict spectrum holes. & $\mathrm{T}$ & I \\
\hline & & HMM & [118] & $\begin{array}{l}\text { extended the NS-HMM method with an expectation maximization based pa- } \\
\text { rameter estimation algorithm. }\end{array}$ & $\mathrm{T}$ & I \\
\hline & Time, Frequency & MC & [210] & $\begin{array}{l}\text { developed a joint spectral-temporal spectrum prediction from incomplete his- } \\
\text { torical observations. }\end{array}$ & $\mathrm{E}$ & $\mathrm{U} / \mathrm{O}$ \\
\hline
\end{tabular}

authors of [209]-[211] proposed various joint two-dimensional spectral-temporal spectrum prediction algorithms by leveraging the low-rank nature of the spectral data. The authors of [213] developed a multi-dimensional (time, frequency, and space) spectral map construction method.
Second, there are some quantitative comparisons among different spectrum inference techniques. Specifically, it was reported in [20] that under the same traffic scenario, an ANN based predictor performs slightly better than the HMM predictor owing to having a flexible number of states and 
TABLE III: Major developments of spectrum inference techniques (Part 2) (T/E: Theoretic/Experimental work; U/O/I: Underlay/Overlay/Interweave).

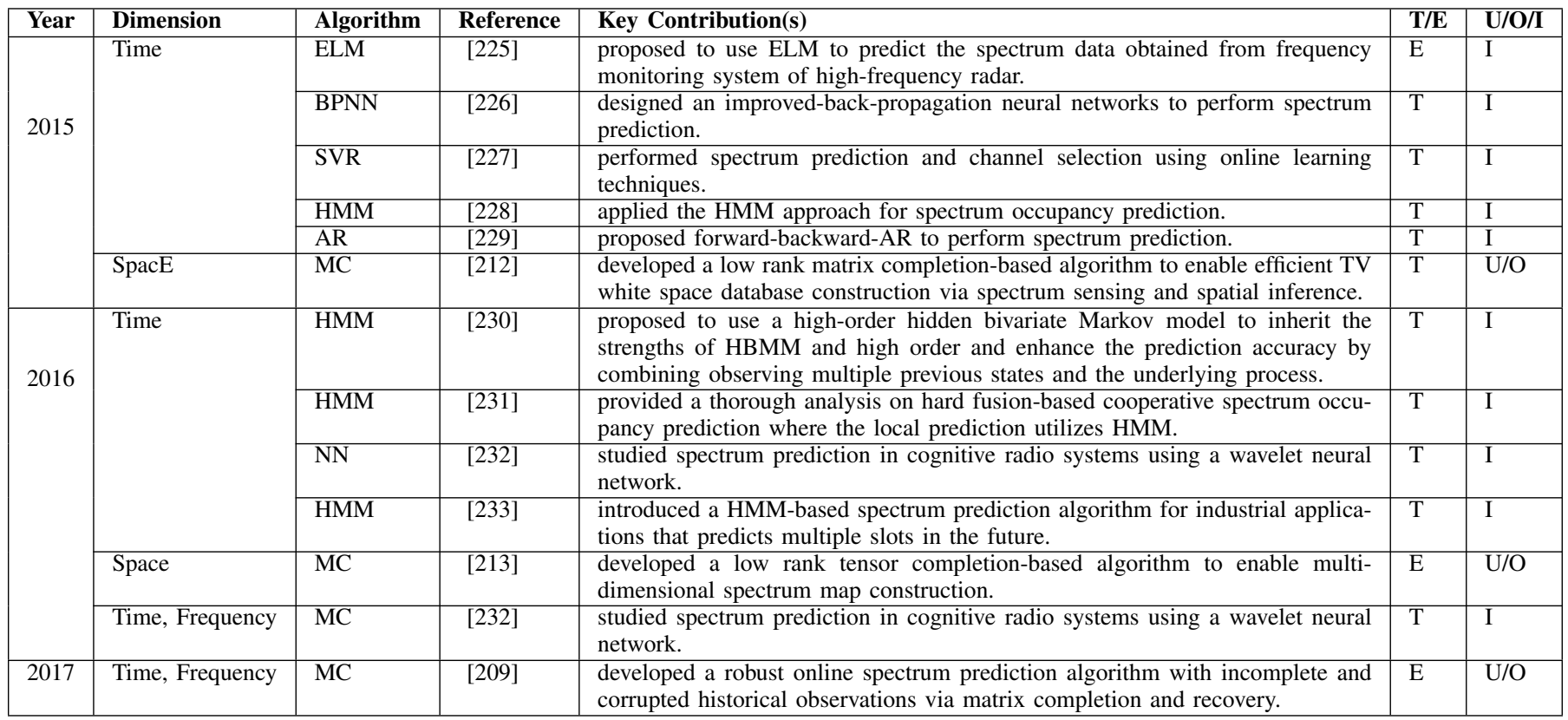

TABLE IV: Discussions on advantages and challenges of various spectrum inference algorithms.

\begin{tabular}{|c|c|c|c|}
\hline \multicolumn{2}{|c|}{ TYPEs } & Advantages & Challenges \\
\hline \multirow[t]{2}{*}{ Markov } & $H M M$ & $\begin{array}{l}\text { 1. solid statistics foundation } \\
\text { 2. robustness in time sequence processing } \\
\text { 3. flexibility in non-stationary scenarios }\end{array}$ & $\begin{array}{l}\text { 1. complex matrix operations and a large number } \\
\text { of previous data needed } \\
\text { 2. large memory } \\
\text { 3. hard to find the optimized number of states } \\
\text { 4. high computational complexity } \\
\text { 5. cannot capture the rich temporal covariance of } \\
\text { activity on the channel at multiple delays } \\
\text { 6. discrete Gaussian models of observations is not } \\
\text { suitable }\end{array}$ \\
\hline & POMDP & adapt to uncertain information in the environment & $\begin{array}{l}\text { 1. difficult to model the environment of dynamics } \\
\text { (estimate the probabilities of action outcomes } \\
\text { and the accuracy of data) } \\
\text { 2. lacking efficient resolution algorithm }\end{array}$ \\
\hline \multirow[t]{2}{*}{$\mathbf{L P}$} & $A R$ & $\begin{array}{l}\text { 1. low complexity for low orders } \\
\text { 2. convergence guaranteed } \\
\text { 3. needs no thresholds }\end{array}$ & $\begin{array}{l}\text { 1. requires some training data } \\
\text { 2. dependent on stationary processes (except ARIMA) } \\
\text { 3. high accumulated errors for high order } \\
\text { 4. computationally expensive for high order }\end{array}$ \\
\hline & ARIMA & 1. able to process non-stationary sequences & $\begin{array}{l}\text { 1. needs considerable statistical skills } \\
\text { 2. not suitable for fast learning }\end{array}$ \\
\hline \multicolumn{2}{|c|}{ FFNN } & $\begin{array}{l}\text { 1. flexibility in non-stationary scenarios } \\
\text { 2. need no priori knowledge of the observed process } \\
\text { distribution } \\
\text { 3. does not need to set parameters } \\
\text { 4. needs no thresholds }\end{array}$ & $\begin{array}{l}\text { 1. computationally expensive training process } \\
\text { 2. hard to identify the optimal number of intermediate } \\
\text { layers and neurons in each layer } \\
\text { 3. large number of free parameters } \\
\text { 4. local minima problem during training }\end{array}$ \\
\hline \multicolumn{2}{|c|}{ FPM } & $\begin{array}{l}\text { 1. robustness in time sequence processing } \\
\text { 2. easy to implement } \\
\text { 3. convergence guaranteed }\end{array}$ & dependent on stationary processes \\
\hline \multicolumn{2}{|c|}{ BIF } & dynamic prediction for even incomplete data & $\begin{array}{l}\text { 1. no feedback } \\
\text { 2. requires the knowledge of distribution }\end{array}$ \\
\hline \multicolumn{2}{|c|}{ SVM } & $\begin{array}{l}\text { 1. flexibility in non-stationary scenario } \\
\text { 2. guarantees to converge to optimal solution } \\
\text { 3. small number of free parameters } \\
\text { 4. computationally efficient }\end{array}$ & $\begin{array}{l}\text { 1. requires prior knowledge of the observed process } \\
\text { distribution } \\
\text { 2. computationally expensive training process }\end{array}$ \\
\hline \multicolumn{2}{|c|}{ LA } & 1. simple structure & increase model complexity \\
\hline
\end{tabular}

a more efficient training mechanism. Although having more intermediate layers in the ANN increases their computational complexity, the prediction results are improved [157]. As expected, there is a tradeoff between the complexity and the accuracy. Moreover, it is reported in [139] that the short- term prediction performance of the ANN based predictor is better than that of the ARIMA predictor, but their performance degrades, when predicting further into the future. The performance of the HMM predictor suffers from two inherent limitations [218]. Since the radio environment is changing 
all the time, the fixed states assumed in Markov modeling fail to capture the rich temporal variations of the channel activities. Another limitation of the HMM aided predictor is that discrete or univariate Gaussian models of observations often fail to adequately characterize the general situations. The POMDP predictor gives full cognizance to random actions, to incomplete information and to noisy observations of the environment, but it may not be able to accurately calculate the probabilities of specific action outcomes [219]. When it comes to pattern mining based schemes, the partial periodic pattern mining predictor is more robust than the HMM predictor owing to extracting more channel state prediction rules, which reduces the probability of spectrum usage prediction compared to traditional frequent-pattern mining [16].

\section{Algorithm Analysis of Spectrum Inference IN CRNS}

\section{A. Performance Metrics}

When evaluating or choosing a prediction algorithm, carefully selected metrics should be considered. Typically the notion of detection probability $\left(P_{d}\right)$ representing the correct prediction of the busy states and the false-alarm probability $\left(P_{f a}\right)$ representing the rate of incorrect prediction of idle states are used for studying the performance of algorithms in spectrum occupancy prediction. A combination of a higher $P_{d}$ and lower $P_{f a}$ suggests a better prediction performance. Some other statistical criteria like the normalized mean square error (NMSE), the mean absolute percentage error (MAPE), and the root mean square error (RMSE) can also be introduced to quantify the adequacy of prediction. In order to demonstrate the advantage of using spectrum prediction, both the percentage improvement in spectrum utilization and the percentage reduction in sensing energy can also be invoked.

Spectrum inference/prediction is an efficient technique complementary to other techniques, such as spectrum sensing, geolocation spectrum database, and radio environment map, for capturing the relevant information about the spectral evolution and identifying spectrum access opportunities. In this subsection, we first briefly present the key ideas of each technique one by one. Then, we discuss the relationships between spectrum inference and the other techniques.

\section{B. Relationship with Spectrum Sensing}

Spectrum sensing determines spectrum state in a passive manner using various signal detection methods. This approach, however, suffers from the hidden node problem because of shadowing [239]. In the past decade, intensive studies have been carried out across the globe to improve the detection performance of spectrum sensing. Excellent survey papers in this direction can be found in [25]-[27]. Briefly, existing studies on spectrum sensing in CRNs can be classified into two groups: quiet-periods-based (see, e.g. [26]) and nonquiet-periods-based spectrum sensing (see, e.g. [291]), also known as spectrum monitoring in the literature. As the name implies, in the former studies, a cognitive radio first spends a time duration (known as quiet period) to perform spectrum sensing and then determines whether to transmit based on the sensing results. By contrast, spectrum monitoring in CRNs is a relatively new technique, where the cognitive radios can continue their communications while simultaneously monitoring the band to detect any transmissions that are initiated by primary radios. More specifically, the spectrum is monitored by the cognitive radio receiver during reception and without quiet periods [290]-[292]. So far, spectrum sensing has not been widely accepted by regulatory bodies to ensure nonharming the primary/licensed users. It is challenging to meet the strict rules required by the regulators from FCC, such as the detection of a primary signal at $-114 \mathrm{dBm}$ by FCC.

\section{Relationship with Geolocation Spectrum Database}

By contrast, according to the regulations of FCC [240], [241], the geolocation spectrum database approach seems to provide a technically feasible and commercially viable solution in the near future. This approach provides a service ensuring that an unlicensed device can inquire the spectrum availability from a geolocation database, which predicts the spectrum availability at any location using propagation modeling combined with terrain data [242], [246]. Based on the guidelines provided by FCC [240], several TV white space database systems have been developed by companies [247][250]. A specific limitation of this approach is that the accuracy of the spectrum availability provided by the geolocation database depends crucially both on the quality of the propagation modeling and on the granularity of the terrain data. To resolve this issue, a data-driven approach is presented in [5] to build a spectrum database by learning the spectrum availability from mobile crowd sensing and big spectrum data analytics. Another limitation of this approach is that the update speed of the spectrum availability provided by the geolocation database is relatively slow (e.g., in the time scale of hours or days).

\section{Relationship with Radio Environment Maps}

The radio environment map (REM), concept was originally proposed by scientists from Virginia Tech [251]-[253], which is a promising tool that provides a practical realization of CRNs, explicitly, it constructs a comprehensive map of the CRN by utilizing multi-domain information from geolocation databases, characteristics of spectrum use, geographical terrain models, propagation environment and regulations [254]. In contrast to the geolocation spectrum database, REM is an advanced knowledge base that stores live multi-domain information on the entities in the network as well as on the environment [255], [256].

Spectrum inference is an efficient technique complementary to the above techniques. Spectrum inference, with spectrum prediction in the time domain as a special case, infers an unknown spectrum state from known spectrum data, by effectively exploiting the inherent statistical correlations of spectrum data in time, frequency and spatial domains. A key distinguishing feature of spectrum inference is that it presents a proactive view of the spectrum state. In terms of the relation with spectrum sensing, spectrum inference can reduce the sensing time and energy consumption [127]. Spectrum inference (e.g., in time domain) can also be fused with spectrum 
sensing to improve the detection performance of spectrum access opportunities [12]. In terms of the relationship with a geolocation spectrum database, spectrum inference (e.g., in spatial domain) can be further used to calibrate the propagation models and to improve the update speed of the database. In terms of its relationship with REM, spectrum inference in multiple domains (i.e., time-frequency-space) can be used to construct dynamic interference maps for each frequency at each location of interest [254], in a proactive and energyefficient manner.

\section{EXPERIMENTATION-BASED APPROACHES IN CRNS}

Although the majority of the existing spectrum inference algorithms are mainly studied from a theoretical perspective, there are some studies that aim for bridging the theory and the practice. In this section, we present discussions regarding real experimentation-based spectrum inference approaches in CRNs. We begin with presenting several well known spectrum-related experimental projects, which serve as the hardware/software basis for the development of spectrum inference algorithms. Then, we briefly comment on the analysis of real-world spectrum measurements. Furthermore, we discuss the experimental results reported in the literature.

\section{A. Spectrum-Related Experimentation Projects}

During the past two decades, quite a few spectrum-related experimental projects have been carried out all over the world, which collected empirical real world spectrum measurements and supported the development of spectrum inference techniques. Excellent surveys and tutorials on the latest advances of worldwide spectrum projects and occupancy measurements can be found in [23] and [24]. In the following, we provide a brief review of several well-known spectrum occupancy evaluation projects to assist the readers.

- ORCA, which stands for Optimization and Rational use of wireless Communication bAnds [294], is a project with the general scientific objective of extensively evaluating the spectrum occupancy in order to study the potential for its exploitation by innovative wireless services. This project developed DTV coverage prediction algorithms by exploiting for example the Longley-Rice propagation model [295], [296].

- WiSHFUL, which stands for Wireless Software and Hardware platforms for Flexible and Unified radio and network controL [297], is a project funded by the European Commission's Horizon 2020 Programme with the following objectives: i) to offer open, flexible and adaptive software and hardware platforms for radio control and network protocol development; ii) to offer portable facilities that can be deployed at any location; iii) to attract and support experimenters for wireless innovation.

- CREW, which stands for Cognitive Radio Experimentation World [298], is a project with the main target of establishing an open federated test platform, which facilitates experimentally-driven research on advanced spectrum sensing, cognitive radio and cognitive networking in view of both horizontal and vertical spectrum sharing in licensed and unlicensed bands. Parts of the research outputs of this project focus on constructing radio environment maps using various spatial inference techniques [299].

- FARAMIR, which stands for Flexible and spectrumAware Radio Access through Measurements and modeling in cognitive Radio systems [300], is a project making CRs a reality, with the main goal of developing techniques for increasing the radio environmental and spectral awareness of future wireless systems. Parts of the research outputs of this project focus on constructing radio environment maps using various statistical inference techniques [301], [302].

\section{B. Predictability Analysis of Real Spectrum Measurements}

With respect to spectrum inference/prediction, there is a fundamental question: To what degree is the spectrum state evolution predictable? In other words, the predictability of spectrum evolution reflects the upper-bound performance of all potential spectrum inference/prediction techniques. When considering the temporal spectrum usage evolution for a given frequency band in a given location as a random time series, the entropy is the most fundamental quantity characterizing the degree of predictability for this random variable. In general, having a lower entropy implies a higher predictability and vice versa. Entropy-based analysis has indeed been introduced in diverse prediction scenarios, such as for the atmosphere [6], in finance [7], for network traffic [8] and for human mobility [9]. The basic idea is that the entropy offers a precise definition of the information content of predictions and it is renowned for its generality due to relying on minimal assumptions concerning the model of the scenario studied.

Recently, in [127], we have invoked an information theoretic methodology of using statistical entropy measures and Fano's inequality to quantify the degree of predictability underlying real-world spectrum measurements. We found that despite the apparent randomness, a remarkable $90 \%$ predictability may be achieved, in real-world RSS dynamics over diverse spectral bands for several popular services, including the classic TV bands, cellular bands, the (Industrial Scientific Medical) ISM bands, etc. Similarly, Olivieri et al. [123] have applied the information theoretic entropy as a measure of predictability in the process of generating the ON- and OFF-period durations. In [124]-[126], the authors have used state-of-the-art multiscale entropy metrics in order to examine the predictability of the spectrum measurement traces recorded as a function of the prediction complexity.

\section{Experimental Spectrum Inference Approaches}

As shown in Tables II and III, although the majority of the existing spectrum inference algorithms are predominately studied from a theoretical perspective, there are some studies in the open literature that implement and test spectrum inference approaches from an experimental perspective.

To the best of the authors' knowledge, [140] is one of the earliest studies of this kind, where the Markovian property 


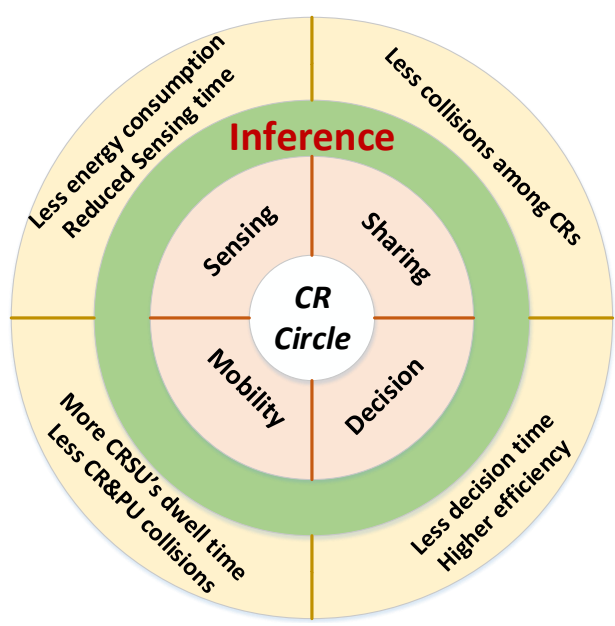

Fig. 9: Illustration of the applications and benefits of spectrum inference in CRNs.

of the spectrum usage has been validated by analyzing realworld measurements in the paging spectrum band (928-948 $\mathrm{MHz}$ ). Experimentally, the HMM is used for predicting the ON-OFF activity of the PU with a prediction accuracy of about $76 \%$. Similarly, Z. Chen, et al [14] verify the HMM method using measured WiFi data instead of artificial data, where the numerical results show that the prediction accuracy for a single device is above $70 \%$, which can be improved by cooperative prediction of multiple devices. In [111], the authors train and validate the HMM model with the aid of real measurements collected from the $14 \mathrm{MHz} \mathrm{HF}$ amateur band, where an average of $10.3 \%$ prediction error rate is achieved.

In [162], a multi-layer perception ANN is applied to forecast the idle or busy state of different channels, where the performances are evaluated for a seven-day spectrum dataset collected in a metro city located in south China and the empirical results show a high prediction accuracy having a mean RMSE as low as 0.04. In [119], based on spectrum measurements ranging from $20 \mathrm{MHz}$ to $3 \mathrm{GHz}$ at four locations in Guangdong province of China, the authors develop a 2-dimensional frequent pattern mining (FPM) algorithm to predict the availability of multiple channels simultaneously, where the numerical results show that the prediction accuracy is higher than $95 \%$ for the GSM900/1800 downlink and for the TV bands.

In [210] the authors develop a joint spectral-temporal prediction from incomplete historical observations, where real measurements of TV bands (614 MHz-698 MHz) are used for quantifying the improvements over the one-dimensional neural network-based temporal prediction scheme. In an extended work [209], the authors develop a robust online spectrum prediction algorithm relying on incomplete and corrupted historical observations, where the real measurements of TV bands, ISM bands and GSM bands are used for demonstrating that the proposed algorithm performs well in the presence of missing data.

\section{Applications of SpeCtrum InfEREnCE IN CRNS}

The specific benefits of cognitive radio networks depend on our ability to infer/predict the unknown/unmeasured spectrum states from known/measured spectrum data. To portray the various applications of the spectrum inference technology, in this section we first review the existing use cases and then we portray spectrum inference in the context of: i) 5th generation mobile communications (5G) spectrum sharing, ii) nextgeneration high-frequency (HF) automatic link establishment (ALE), and iii) several other potential applications in various future wireless networks, such as cognitive smart grid networks, cognitive radio sensor networks, cognitive cellular networks $(\mathrm{CCN})$, and cognitive machine-to-machine communications (CM2M).

Based on the spectrum management framework proposed in [4], the existing applications of spectrum prediction in CRNs can be classified into four basic categories, namely, spectrum sensing, spectrum decisions, spectrum evolution upon handover and spectrum sharing [21]. The corresponding example applications of the inference algorithms discussed in Section VI on each category are summarized in Table V. Moreover, the applications and benefits of exploiting spectrum inference/prediction in CRNs are presented in Fig. 9, Table VI and briefly summarized as Section VI-A to Section VI-D.

\section{A. Spectrum Inference for Sensing}

Spectrum sensing is a critical aspect of spectrum inference applications that aim to explore idle spectrum slots. In the emerging spectrum sensing paradigms in the time, frequency and spatial dimensions, inference techniques are widely used to infer the most possible vacant channels for improving the detection performance and reducing the energy and time consumption of sensing.

In terms of spectrum inference in the time domain for spectrum sensing, refs. [15], [93], [128], [129], [139], [187], [220] use various linear prediction techniques, such as AR, MA, ARMA and ARIMA to perform spectrum prediction, where the output is used to improve the sensing accuracy and reduce the sensing cost. In parallel, Markov models such as HMM [14], [17], [20], [95], [111], [118], [140], [142], [144], [146], [218], [221], [222] and POMDP [153] are also widely used to perform similar tasks. These kinds of models work well under the assumption of memoryless or Markov property existing in the spectrum state evolution. That is, the future state depends only on the relevant information about the current, not on information from further in the past. ANN models are another kind of techniques that are widely investigated (see, e.g. [20], [119], [139], [158], [160]-[162], [167], [168], [178], [183], [223], [224]), which show relatively better prediction accuracy over other mondels. In addition, pattern mining methods [119], [216], BIF methods [17], [187] and SVM methods [190], [191] are also investigated.

In terms of spectrum inference in the frequency domain for spectrum sensing, the spectral occupancy correlation between adjacent channels has been evaluated by experiments in [194][196]. Related studies are well dedicated to inferring the states of other channels in the light of already acquired sensing or 
TABLE V: Existing applications of various spectrum inference algorithms.

\begin{tabular}{|c|c|c|c|c|c|c|}
\hline \multicolumn{3}{|c|}{$\begin{array}{ll}\text { Methodology } & \text { Application } \\
\end{array}$} & For spectrum sensing & For spectrum decision & For spectrum mobility & For spectrum sharing \\
\hline \multirow{13}{*}{$\begin{array}{l}\text { Time } \\
\text { Inference }\end{array}$} & \multirow{4}{*}{$L P$} & $A R$ & {$[15],[93],[128],[187]$} & & & \\
\hline & & & {$[128],[129]$} & & & \\
\hline & & ARMA & {$[128]$} & & & \\
\hline & & ARIMA & [139], [220] & [220] & & \\
\hline & \multirow[t]{2}{*}{$\begin{array}{l}\text { Markov } \\
\text { Models }\end{array}$} & $H M M$ & $\begin{array}{c}\text { [14], }[17],[20], \quad[95], \\
{[111], \quad[118],} \\
{[142],} \\
{[218],[22144],[222]}\end{array}$ & [17], [95], [111], [221] & & \\
\hline & & $P O M D P$ & {$[153]$} & {$[153]$} & & \\
\hline & \multirow[t]{2}{*}{ ANNs } & FFNNS & $\begin{array}{l}{[20], \quad[139], \quad[158],} \\
{[160]-[162],} \\
{[168],[223],[234]}\end{array}$ & {$[161],[167],[168]$} & & \\
\hline & & FBNNs & {$[178],[183],[224]$} & {$[178]$} & & \\
\hline & \multirow[b]{2}{*}{$P M$} & $F P M$ & & [119] & & \\
\hline & & $P P P M$ & [216] & & & \\
\hline & \multirow{2}{*}{\multicolumn{2}{|c|}{$B I F$}} & {$[17],[187]$} & {$[17],[208]$} & [174] & \\
\hline & \multirow{2}{*}{\multicolumn{2}{|c|}{$\frac{D N A}{L A}$}} & [190], [191] & {$[190],[191]$} & {$[202],[203]$} & \\
\hline & & & {$[157]$} & {$[157]$} & & \\
\hline \multicolumn{3}{|c|}{ Frequency Inference } & $\begin{array}{l}\text { [12], [93], [119], [192], } \\
{[193],[195]}\end{array}$ & [119], [198], [199] & [175] & \\
\hline \multicolumn{3}{|c|}{ Geographic Inference } & {$[212],[213]$} & [198], [201] & [204], [205] & $\begin{array}{c}{[5],[18],[176],[200]} \\
{[238]}\end{array}$ \\
\hline
\end{tabular}

TABLE VI: A List of Reported Benefits/Gains of Exploiting Spectrum Inference in CRNs.

\begin{tabular}{|c|c|c|c|}
\hline Reference & Domain & Application & Benefits/Gains \\
\hline$[234]$ & Time & Sensing & $\begin{array}{l}\text { Spectrum prediction is designed to select the most likely idle channel for sensing and } \\
\text { brings } 10-30 \% \text { throughput improvement. }\end{array}$ \\
\hline [237] & Time & Decision & $\begin{array}{l}\text { Spectrum prediction is introduced to be fuzed with sensing to improve the detection of } \\
\text { PU and enhance the throughput as large as } 50 \% \text {. }\end{array}$ \\
\hline$[192]$ & Time, frequency & Sensing & $\begin{array}{l}\text { Spectral correlation is introduced to prediction to decrease the time consumption in } \\
\text { spectrum sensing and brings roughly } 20 \% \text { throughput improvement. }\end{array}$ \\
\hline$[12]$ & Time, frequency & Sensing & $\begin{array}{l}\text { Spectrum prediction is introduced to select channels for sensing only from the channels } \\
\text { predicted to be idle and brings } 5-30 \% \text { throughput improvement when the probability } \\
\text { of wrong prediction is no larger than } 0.2 \text {. }\end{array}$ \\
\hline$[236]$ & Time, frequency & Sensing & $\begin{array}{l}\text { Spectrum prediction is introduced to rank channels according to the availability and } \\
\text { quality and brings about } 15 \% \text { reduction of the link establishment time. }\end{array}$ \\
\hline$[5]$ & Space & Sharing & $\begin{array}{l}\text { Compared with the traditional propagation model-based approach, spectrum inference } \\
\text { in spatial domain brings roughly } 20 \% \text { improved spatial reuse between the PU and the } \\
\text { CRs and } 15 \% \text { reduced interference to the PU. }\end{array}$ \\
\hline [212] & Space & Sensing & $\begin{array}{l}\text { Spatial inference is invoked as a complimentary method of spectrum sensing for } \\
\text { enabling efficient TV white space database construction with a } 2-6 \mathrm{~dB} \text { gain in terms } \\
\text { of root square error, compared with the traditional approach. }\end{array}$ \\
\hline$[175]$ & Time & Mobility & $\begin{array}{l}\text { Spectrum prediction and monitoring are jointly used via OR/AND fusion to improve the } \\
\text { perform of spectrum mobility/handoff, which brings } 5-25 \% \text { throughput improvement } \\
\text { by reducing the resource wastage. }\end{array}$ \\
\hline [205] & Space & Mobility & $\begin{array}{l}\text { Moderate accuracy predictors improve routing reliability and bandwidth efficiency by } \\
11 \% \text { and } 8 \% \text {, respectively. }\end{array}$ \\
\hline
\end{tabular}

predicted results [93], [119], [192], [193]. Specifically, the authors of [192] exploited the spectral correlations for inferring the availabilities of other channels in order to improve the throughput. In [193], predicting the states of unsensed channels is formulated as a matrix completion problem and the technique of belief propagation (BP) is applied to fill the matrix with predicted states. Yin et al. [119] has proposed a frequency pattern matching algorithm operating in twodimensions (FPM-2D) for spectrum inference, which searches through all relevant 2D patterns. Similarly, frequency domain correlation techniques were also introduced in [93] by modeling the neighboring channels in pairs, where the classic AR models which do not need a priori knowledge on the communication environment were also employed to reduce the complexity.

The existing studies on spectrum inference in the spatial domain for spectrum sensing are relatively limited. In [212], a matrix completion-based algorithm is developed for TV white space database construction via joint spectrum sensing in time domain and spectrum inference in spatial domain. In [213], the authors propose the concept of spectrum tensor and develop a multi-dimensional (including, time, frequency, and space) spectrum inference algorithm for spectrum map construction by invoking the recent advances in tensor completion.

\section{B. Spectrum Inference for Decision}

Spectrum inference for decision has been investigated in various aspects such as, centralized spectrum allocation, decentralized channel selection, physical layer rate adaption, dynamic spectrum access, to name just a few. In the following, we introduce several representative examples. 
Akbar and Tranter in [95] proposed to use the HMM based prediction algorithm for dynamic spectrum allocation, where the superiority in terms of system throughput is presented by comparing with traditional CSMA based algorithms. Qin et al in [199] and Melián-Gutiérreza et al in [111] respectively studied the problem of spectrum inference-based channel selection in the HF spectrum. The authors of [153] proposed an approach for channel state prediction based on POMDP by finding the optimal policy that maximizes some aspect of the reward. The authors in [167], [168] proposed to use neural networks based cognitive controller for dynamic channel selection and adaptation. In [178], Elman recurrent neural networks was used for radio frequency multivariate time series modelling in order to predict the spectral evolution, which leads to intelligent $\mathrm{CR}$ decisions for exploiting the expected spectrum opportunities, optimized spectrum usage and interference avoidance.

\section{Spectrum Inference for Mobility}

The mobility terminology in the CRNs has double meanings. On the one hand, it refers to 'spectrum mobility or spectrum handoffs' from one spectral band to another, for example, due to the appearance of PUs or owing to interference avoidance for other CRs. One the other hand, the 'mobility of CRs and/or PUs', for example in vehicular CRNs, may also affect the surrounding geographical spectral environment in terms of imposing additional interference, hence changing channel conditions and spectrum availability, etc.

An excellent survey paper on spectrum mobility in CRNs was conceived by [170], which starts from the consideration that in highly dynamic environments, CR-aided communication is often interrupted and hence spectrum mobility is recognized as a pivotal feature of enabling continuous CR data transmission, by transferring ongoing sessions to an idle channel. A recent classification and survey was provided by [171]. In [172], a Poisson distribution based model of spectral resources relying on a cross-layer spectrum handoff protocol optimized for the minimum expected transmission time was developed for cognitive LTE networks. However, the fluctuating nature of the available spectrum makes it difficult to support seamless CR communications. To address this problem, a spectrum-aware mobility management scheme is proposed in [173] for CR cellular networks, where a unified mobility management framework is developed for supporting the diverse mobility patterns in CR networks, which consists of spectrum mobility management, user mobility management and inter-cell resource balancing.

Over the years, most of the existing studies have been focused on so-called reactive spectrum mobility, where the CR switches its communication once a PU becomes active, where the detection of the PU relies either on spectrum sensing or on monitoring. However, there are also some emerging proactive spectrum mobility solutions, based on prediction or inference techniques. For instance, Bütün et al. [205] propose a static neighbor graph (SNG) algorithm for predicting the mobility of cognitive users. Wang et al. [202] proposed a support vector machine (SVM) based spectrum mobility prediction algorithm for mobile CRNs. In [203], the SVM was invoked both for predicting the handoff point and the idle channels with a high precision. In addition to the spectrum availability, the link availability between the CRs was also inferred by [204], where by invoking this novel approach, a reliable path can be found for dynamic routing in CRNs. In [205], a location predictor is proposed, where the historical changes of the PU's geographic locations are represented by a directed graph having weighted edges. Once a spectrum occupancy prediction is requested, all the edges originating from the starting point are listed and then the destination is predicted according to the approximately calculated maximum weights. Huang et al. [174] propose a Bayesian inference-based prediction algorithm for spotting the specific channel that is most likely to be available for the CR-aided vehicular ad-hoc networks, where the most critical challenges are the high-speed mobility of vehicles and the dynamically-fluctuating channel availability. As a further advance, P. Thakur et al. [175] propose a proactive spectrum prediction technique, where the emergence of $\mathrm{PU}$ is predicted before its true emergence, in order to avoid dropping even a single packet.

\section{Spectrum Inference for Sharing}

In the context of spectrum sharing, there are different understandings in the literature. In the generalized sense, the concept of spectrum sharing is inter-changeable with the concepts of dynamic spectrum access or cognitive radio, which consists of three paradigms of spectrum usage: underlay, overlay and interweave [120]. This kind of understanding of spectrum sharing makes its meaning too board to cover every aspects of CRNs. On the other hand, in the narrow sense, spectrum sharing focuses on the underlay mode, which allows CRs to operate in the same band at the same time if the mutual interference among them is below a tolerant threshold. Spectrum inference for sharing is invoked for supporting/facilitating the coexistence of CRs with the PUs.

As shown in Table $\mathrm{V}$, the research on inference-based spectrum sharing is relatively limited and mainly focused on spatial domain [5], [18], [176], [200]. In [5], a systematic approach is developed to enable efficient spectrum sharing between the PU (i.e., TV receivers) and the CR (e.g., smart phone, tablet, mobile vehicles, etc) where matrix completionbased spectrum inference technique is invoked to serve as a spatial interpolator of unmeasured spectrum data. In [18], the authors propose to predict the contour and service areas of the PU for enabling unlicensed CR systems operating in the TV white space without producing harmful interference to the PU receivers. Recently, Kim and Giannakis in [200] have proposed a dictionary learning framework to predict the interference power levels in various locations for enabling harmonious spectrum sharing between the PU and the CR. In [176], the authors formulate the adaptive vehicular data piping problem for dynamic spectrum sharing as a coalitional formation game and propose a near optimal coalitional formation approach for enabling vehicular data pipe selection partition in a distributed way. 


\section{E. Potential Use Case I: Spectrum Inference for Supporting $5 G$ Spectrum Sharing}

Radio spectrum sharing is an essential topic for $5 \mathrm{G}$ wireless communications [259], [260]. The explosive growth of data rates offered by smart phones, tablets, laptops, vehicles and many other wireless devices is about to overwhelm the allocated 2 G-3G-4G radio spectrum. Spectrum sharing is emerging as an affordable, near-term method of meeting the $5 \mathrm{G}$ radio spectrum shortage and increasing the radio access network capacities for supporting 5G content delivery [261]. Spectrum sharing may occur between an incumbent user (e.g., commercial TV or public radars) and a secondary $5 \mathrm{G}$ user, when a $5 \mathrm{G}$ device uses a spectrum band allocated for an incumbent usage in a geographic place, time and RF channel that the incumbent is not using, subject to the tolerable interference imposed on the incumbent [5].

Fig. 10 presents a vision of spectrum inference/predictionbased spectrum sharing conceived for $5 \mathrm{G}$ wireless communications. Specifically, in the basic operation cycle printed in light color, the spectrum sensing module infers the observable RF stimuli from the radio environment and outputs the predicted RSS in various spectrum bands of the current time slot. Based on the input from spectrum sensing, the spectrum sharing module activates spectrum reuse and interference control to ensure the safe coexistence between commercial or public incumbent users and secondary $5 \mathrm{G}$ users. It may be deemed inevitable for $5 \mathrm{G}$ devices to explore and exploit the benefits of multiple (non-continuous) spectrum bands of a wide range, spanning from a few hundred $\mathrm{MHz}$ in the VHF/UHF band to the 30-300 GHz millimeter-wave length band. In this case, predictive schemes are expected to obtain the RSS of such wide spectrum bands and to enable wideband spectrum sharing in a timely and cost-efficient manner based on time domain or joint time-frequency domains spectrum inference algorithms discussed in Section IV. Specifically, in Fig. 10, a spectrum inference/prediction module is introduced, which infers the future RSS based on the historical RSS data acquired by spectrum sensing, which can further enhance $5 \mathrm{G}$ spectrum sharing for example by,

- supporting adaptive PHY-layer spectrum sensing, i.e. adaptive optimization of the sensing parameters, such as the sensing time duration in each time slot,

- facilitating resource-efficient MAC-layer spectrum sensing, for example by reducing the number of time-slots required for multi-band sequential sensing as well as scheduling and by reducing the energy consumption of multi-sensor cooperative sensing as well as scheduling,

- supporting high-data-rate spectrum sharing by combining the outputs of spectrum sensing and spectrum prediction, for example by guiding the selection of spectrum bands of high channel quality in carrier aggregation.

Specifically, in 5G systems, prediction-based spectrum sharing can be arranged at the base stations (BSs) of macro/small cells and at the access points (APs) of WLANs in a centralized manner as well as more aggressively, by the autonomous user equipment in a self-organized manner by relying on game theory and graph theory.
F. Potential Use Case II: Spectrum Inference for NextGeneration HF Automatic Link Establishment

High-frequency (HF) radio, also known as short-wave radio [262], operating in the $1.5-30 \mathrm{MHz}$ spectrum band, is now widely used, not only by the amateur community, but also by worldwide governmental and non-governmental agencies as an alternative to satellites for over-the-horizon wireless communications. Typical application scenarios of HF radios include ships at sea, aircraft out of range of line-of-sight radio networks, disaster areas where the terrestrial communications infrastructure has been destroyed, and distant regions lacking other communications, to name just a few.

One of the key challenges in using HF communications is finding a frequency that will support the desired teletraffic from a transmitter to a receiver. The reasons behind this challenge are mainly two-fold: Firstly, over-the-horizon HF communications often use skywave propagation paths provided by ionospheric refraction, which physically makes the window of usable frequencies time-varying throughout the day, the season, the subspot cycle, the weather environment, and the radio locations, etc [263], which is quite different from terrestrial wireless communications, such as cellular and WLAN systems. Second, there are many governmental and non-governmental HF radio systems in the HF band, which make it a nontrivial task for each transmission to find a frequency without interfering with other users [264].

To tackle the above critical challenge, ALE [263] is wellrecognized as a promising technology, which automates the process of finding a usable frequency and setting up links between two or more radios. Since the late 1970s, three generations of automatic link establishment techniques have been developed. Briefly, the first generation ALE was independently developed by different manufactures to automatically identify suitable transmission frequencies using microprocessors, instead of the original manual operation. The second generation ALE focused on standardized, interoperable HF radio systems, relying on the standards such as MIL-STD-188-141A and FED-STD-1045. The third generation ALE operates at a lower SNR, carries more traffic and supports larger networks.

The ALEs of the first three generations are in essence narrowband ALE operating over $3 \mathrm{kHz}$ HF channels. However, in recent years we have seen an increasing demand for higherdata-rate transmission over HF links, supporting services ranging from voice and low-speed data to real-time video over HF skywave channels from aircraft, which motivates us to develop wideband ALE operating over HF channels wider than $3 \mathrm{kHz}$, and up to $24 \mathrm{kHz}$ [263]. Wideband ALE, also termed as fourth generation (4G) ALE, introduces an increased bandwidth flexibility as well as additional automated capabilities: i) detect and characterize the occupancy or interference within a wideband channel, and ii) coordinate the allocation of the clear subchannel.

The literature [264]-[267] reports on the potential application of cognitive radio techniques in 4G ALE, where consensus ensures that spectrum sensing will indeed be used for HF radios for detecting the occupancy or interference within any portion of channels up to $24 \mathrm{kHz}$. Based on data link quality 


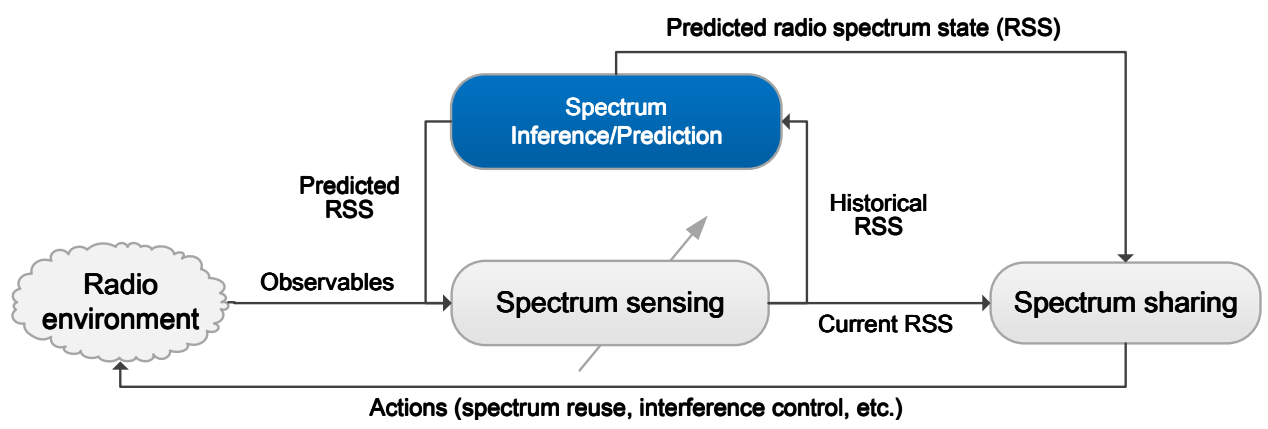

Fig. 10: Spectrum inference for spectrum sharing in $5 \mathrm{G}$ wireless communication systems. The basic operation cycle is in light color and the functionality of spectrum inference/prediction is in dark color.

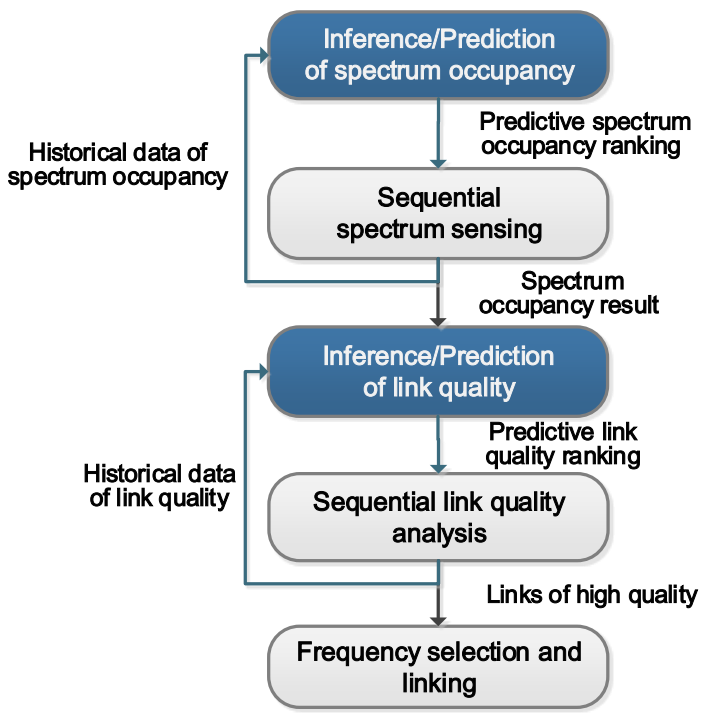

Fig. 11: A vision of spectrum inference/prediction-based wideband $4 \mathrm{G}$ HF ALE. The basic operating cycle is in light color and the functionality of spectrum inference/prediction is in dark color.

analysis (LQA), the associated frequency selection algorithm will be invoked for determining which channel(s) will be used for data transmission. The research and development of $4 \mathrm{G}$ ALE is still in its infancy [268].

Fig. 11 presents a vision of spectrum inference/predictionbased wideband 4G ALE. Specifically, in the basic operating cycle printed in light color, firstly sequential spectrum sensing is performed for detecting the occupancy or interference within each channel. Based on the output of spectrum sensing, sequential LQA is then performed on the particular channels, which are deemed to be unoccupied. Based on the LQA result, the frequency band(s) having a high quality will be selected as the active transmission link. One of the main problems of the basic operation cycle for wideband ALE is that the amount of time required both by the process of spectrum sensing and LQA increases proportionally with the number of frequencies sensed and analyzed. To reduce the associated delay, spectrum inference/prediction may be invoked as seen in Fig. 11. Specifically, spectrum inference can be employed in two specific ways:
- Inference/Prediction of spectrum occupancy, which outputs the predicted spectrum occupancy ranking. Based on this output, the specific frequency of the particular bands exhibiting a higher probability of being unoccupied will be sensed first in the following process.

- Inference/Prediction of link quality, which outputs the predicted link quality ranking. Based on this output, the specific frequency of the links having a higher predicted link quality will be firstly selected for LQA.

We note that in the basic operating cycle of wideband $4 \mathrm{G}$ ALE, the specific order in which spectrum sensing and LQA are carried out is generally random or predefined. By contrast, the introduction of spectrum inference/prediction is capable of providing an informative guidance considering the sensing order and LQA order. Therefore, the link establishment time is expected to decrease in $4 \mathrm{G}$ ALE, provided that the spectrum inference techniques summarized in the previous sections are invoked. To enable the vision shown in Fig. 11, time domain or joint time-frequency domains spectrum inference algorithms discussed in Section IV can be used.

\section{G. Other Use Cases: The Applications of Spectrum Inference for Various Future Wireless Networks}

In addition to $5 \mathrm{G}$ and $\mathrm{HF}$ communications, there are many other potential applications of spectrum inference for various future wireless networks, such as cognitive smart grid networks (CSGN) [269], [270], cognitive radio sensor networks (CRSN) [271]-[274], cognitive cellular networks (CCN) [275]-[277], and cognitive machine-to-machine communications (CM2M) [278].

Specifically, in cognitive radio-enabled smart grid network$\mathrm{s}$, spectrum sensing and spectrum inference techniques are promising methods of providing timely smart grid wireless communications by utilizing all available spectrum resources. Besides the prediction of spectrum state evolution, various statistical inference techniques can also be utilized to predict the system state of the smart grid itself and the potential faults resulting from environmental disasters, cyber attacks and mechanical failures [269].

In CRSNs, wireless sensor networks benefit from the advantage of cognitive radio to overcome the spectrum scarcity by enabling dynamic spectrum access [271]. Among others, the limited energy/power supply and processing capability of 
wireless sensors impose additional constraints on the utilization of various cognitive radio techniques. More specifically, the design of spectrum inference in CRSNs should carefully balance the tradeoffs among the inference accuracy, the computational complexity, and the memory requirements.

In future CCNs, spectrum sharing-oriented cognitive radio techniques can be used to improve the spectrum utilization and to meet the dramatic increase of wireless data rate requirements. Most of the studies on spectrum sharing in future CCNs assume that the network will be i) multi-tier, including macro cells, small cells such as micro cells and pico cells, and device-to-device or machine-to-machine communications [275]; ii) multi-band, including both licensed and unlicensed bands [276]; and iii) software-defined, relying on various intelligent learning or data mining algorithms operated in the cloud radio access networks [277]. Inference of the spectrum usage state and the users' content demand will facilitate more proactive and efficient network operations.

\section{OPEN ISSUES AND RESEARCH TRENDS}

Although a number of studies have been carried out on spectrum inference in CRNs, this research topic has a great potential for future investigation. Actually, there are still a number of unsolved challenges waiting for solutions. Based on the above survey and tutorial, this section presents a range of potential open issues and future research trends as follows.

\section{A. Fundamental Performance Limits of Spectrum Inference}

First of all, the fundamental performance limits on spectrum inference are still unknown. Most existing work on spectrum inference focus on applying various statistical inference techniques for capturing the spectrum state's evolution. However, to the best of our knowledge, there is a paucity of studies focusing on the theoretical performance analysis of spectrum inference. Just as the Shannon capacity gives the upper bound of various modulation and coding schemes, there should be fundamental performance limits for the various spectrum inference techniques. These fundamental performance limits can guide the further development of spectrum inference algorithms.

As mentioned above, recently, Olivieri et al. [123] have applied the information theoretic entropy as a measure of predictability in the process of generating the ON- and OFFperiod durations. In [124]-[126], the authors have used the multi-scale entropy, in order to examine both the complexity and the predictability of the spectrum measurement traces recorded. In [127], from an information theory perspective, the authors have introduced a methodology of using statistical entropy measures and Fano inequality to quantify the degree of predictability underlying real-world spectrum measurements. However, there is no commonly accepted limit on spectrum inference in the literature so far. Moreover, most of them have focused on the performance limits of time-domain spectrum prediction. No work has considered the limits of joint multidomain spectrum inference, intuitively, which is expected to have a higher performance upper bound.

\section{B. Spectrum Inference in Various Domains}

Besides the time, frequency and spatial domains, in other dimensions such as the code- and angular-domain, to the best of our knowledge, inference/prediction of spectrum usage has not been proposed. Once the system becomes aware of the spreading code that the PU is going to use, the CRs become capable of choosing orthogonal codes to simultaneously transmit information with little or no interference. In the context of multiple input multiple output (MIMO) techniques, the PUs may transmit data within a narrow beam in a specific direction [282]. This provides opportunities for CRs to simultaneously transmit over the same frequency band in different directions. Inference/prediction techniques may help the CRs to infer the PU's transmit phase trajectory and preshift the phase accordingly.

Moreover, most of the existing spectrum inference studies focus on single domain spectrum inference, more specifically, time-domain spectrum prediction, but only very limited work consider joint multi-domain spectrum inference. Actually, no individual spectrum data exists in isolation, there are inherent correlations between this data and its neighbors in time domain, frequency domain, and space domain. Consequently, joint multi-domain spectrum inference is an active research direction, which is expected to bring more comprehensive, accurate and reliable spectrum information.

\section{Spectrum Inference in Various Bands}

Although there have been extensive spectrum measurement campaigns all over the world during the past decade, the majority of the efforts have been focused on TV bands to identify TV white spaces (see e.g. [86] and [87]), which to some extent represent the responses to FCC's spectrum policy task force in 2002 [57] and to FCC's adoption rule for unlicensed use of television white spaces around 2010 [240]. There is an urgent need to perform spectrum measurements and inference, in order to document the usage of spectrum bands that have attracted the recent interest of the regulatory bodies. For instance, FCC issued a report and order in 2015, adopting rules for the commercial use of $150 \mathrm{MHz}$ of spectrum in the $3550-3700 \mathrm{MHz}$ band (known as $3.5 \mathrm{GHz}$ band), where the primary user is the US Department of Defense (DoD) radar systems. There are relatively few studies on the spectrum measurements in the new bands [285]-[287]. Interestingly, spectrum sharing between the incumbent radar systems and the Internet of Things devices or secondary WiFi networks have been recently studied in [287]-[289].

\section{Cooperative Spectrum Inference}

In scenarios where there are multiple PUs and multiple CRs, cooperative spectrum inference among CRs is a promising research direction. Cooperative spectrum inference can benefit from multi-user diversity gains since different CRs may face different shadowing and fading environments and have different spectrum consequences for inference. Furthermore, cooperative spectrum inference can enable different CRs to perform different inference subtasks, which may both reduce the computational complexity and the inference delay. 
Moreover, the geographical movements as well as the data transmission trends of the CRs could be better predicted by cooperative schemes. There are several studies in this direction [258], [280], [281].

\section{E. Deep Spectrum Inference}

With the escalation of spectrum demand, there is a pressing need to recognize, classify and to activate the available bands. In fields like public health, economic development and climate forecasting, data mining has shown a beneficial predictive power [279]. Similar success may be anticipated in the radio environment conceiving the required transmission power, spectrum state, PU/CR location, as long as there is sufficient training data. To handle the flood of spectrum data, recent advances in artificial intelligence techniques, like deep learning and reinforcement learning [283], [284], are promising tools for improving the spectrum inference performance.

\section{F. Spectrum Inference for Sensing}

There are a number of interesting directions of exploiting spectrum inference for sensing. Firstly, as is known, wireless spectrum has a (time, frequency, space, etc) multi-domain space. Due to either the hardware limitations or sensor deployment cost, spectrum sensing can only capture the state of a partial of the spectrum space. To obtain a whole picture of the multi-domain spectrum space, spectrum inference can help to fill the unmeasured space via acting as an interpolator. Second, to find idle spectrum from a large number of candidate bands, the order of spectrum sensing is vital to minimize the sensing time overhead. Spectrum inference can provide a predictive input to spectrum sensing for enabling a better sensing order. In addition, spectrum inference can output a complementary spectrum state estimation to spectrum sensing. It's possible to fuze the output of spectrum inference and spectrum sensing to get a more accurate state estimation.

\section{G. Spectrum Inference for Sharing}

In the context of spectrum sharing, spectrum inference is invoked for supporting/facilitating the coexistence of CRs and PUs. The current research on inference-based spectrum sharing is limited and mainly focused on spatial domain [5], [18], [176], [200], where the inference of spatial signal coverage is used to improve the spatial reuse. One interesting direction is to jointly predict/inference the spectrum demand of CRs and the spectrum activities of PUs for proactively coordinating the spectrum sharing among them. Another direction is to predict/inference the statistical channel gains of CR links, PU links and CR-PU interference links, which can facilitate the resource allocation, especially when the channel gains cannot be obtained due to various practical constraints.

\section{H. Spectrum Inference for Mobility}

As mentioned above, spectrum mobility in the CRNs has a twin interpretation. On the one hand, it refers to spectral handoff from one band to another, due to the appearance of PUs or interference avoidance. One the other hand, the mobility of CRs and/or PUs, for example, in vehicular CRN$\mathrm{s}$, may also affect the geographically surrounding spectrum environment in terms of imposing additional interference, changing channel conditions and spectrum availability, etc. Spectrum inference associated with in the former case has been extensively studied. However, in the latter case, there are only a few contributions.

Supporting high-throughput vehicular communications is important for safety applications, traffic management and mobile Internet access. One promising scenario of spectrum inference for mobility can be found in railway, highway or subway based cognitive communications, where the mobility trajectory is fixed and thus the spectrum demand may be relatively regular for inference. One interesting research direction is to jointly consider the spectrum mobility with the user mobility since the spectrum evolution patterns are generally determined by the human's usage of radio spectrum. Moreover, it is reported in [293] that a $93 \%$ potential predictability of human mobility can be expected, which in-turn can be exploited for supporting accurate spectrum inference.

\section{Spectrum Inference for Decision}

As mentioned above, spectrum inference for decision has been extensively investigated in various aspects such as, centralized spectrum allocation, decentralized channel selection, physical layer rate adaption, dynamic spectrum access, to name just a few. There are relatively few open issues found on this topic. However, one direction is to design demos or systems that utilizes the specific techniques with the well known example as DAPRA's spectrum challenges.

\section{J. Applications of Spectrum Inference}

Last but not least, more investigations on specific applications of spectrum inference techniques is also a fruitful research direction. Although there are several common rules for various applications, the requirements of spectrum inference in different application scenarios are rather diverse. For example, the spectrum occupancy state in TV bands changes relatively slowly on a time-scale of several hours, while the spectrum occupancy state in cellular bands or WiFi bands changes within several milliseconds. Considering the fact that the outdated prediction or inference is useless, the tolerance to time delay in the corresponding spectrum inference is significantly different, which imposes different design constraints on the specific inference algorithms.

\section{CONCLUSION}

Spectrum inference is a promising technique of improving the spectrum exploitation in cognitive radio networks. In this paper, we reviewed the recent advances in spectrum inference based on an extensive study of the existing literature. We first introduced the preliminaries of spectrum inference, including the sources of spectrum data, the models of spectrum usage and the predictability of spectrum evolution. Then we explored various spectrum inference algorithms from a time-frequencyspatial domain perspective and presented an in-depth tutorial. 
We proceeded by offering a comparative analysis of the advantages and challenges of various spectrum inference techniques. Additionally, we reviewed the applications of spectrum inference both in existing and in future wireless networks, including 5G cellular communications, next-generation HF communications, cognitive smart grid networks, cognitive radio sensor networks, etc. We also highlighted a range of open issues and research trends influencing the actual deployment of spectrum inference. We conclude that the main goal of the existing and forthcoming studies on spectrum inference in CRNs is to achieve a compromise amongst the conflicting objectives of improving the prediction accuracy, reducing its computational complexity and memory requirement. This forms a fruitful research area. Our hope is that this paper, with its interdisciplinary perspective, will stimulate the research and development of spectrum inference in future wireless networks.

\section{ACKNOWLEDGEMENT}

The authors thank the Editor and the Reviewers for their helpful comments and constructive suggestions, which have helped us significantly improve this work. The authors also thank Ms. Jing Zhang, Ms. Mengyun Tang and Mr. Guangming Nie in collecting and formatting parts of the references.

\section{REFERENCES}

[1] FCC, "Notice of proposed rule making and order," ET Docket No. 03 322, Nov. 2003.

[2] J. Mitola, "Cognitive radio: An integrated agent architecture for software defined radio," Ph.D. dissertation, KTH Royal Inst. Technol., Stockholm, Sweden, 2000.

[3] S. Haykin, "Cognitive radio: Brain-empowered wireless communications," IEEE J. Sel. Areas Commun., vol. 23, no. 2, pp. 201-220, 2005.

[4] I. F. Akyildiz, L. Won-Yeol, M. C. Vuran, and S. Mohanty, "A survey on spectrum management in cognitive radio networks," IEEE Commun. Mag., vol. 46, no. 4, pp. 40-48, 2008.

[5] G. Ding, J. Wang, Q. Wu, et al., "Cellular-base-station assisted deviceto-device communications in TV white space," IEEE J. Sel. Areas Commun., vol. 34, no. 1, pp. 107-121, 2016.

[6] R. Kleeman, A. J. Majda, and I. Timofeyev, "Quantifying predictability in a model with statistical features of the atmosphere," Proceedings of the National Academy of Sciences, vol. 99, no. 24, pp. 15 291-15 296, 2002.

[7] N. Navet, and S. H. Chen, "On predictability and profitability: Would gp induced trading rules be sensitive to the observed entropy of time series?" Natural Computing in Computational Finance, vol. 100, pp. 197-210, 2008

[8] R. Li, Z. Zhao, X. Zhou, J. Palicot, and H. Zhang, "The prediction analysis of cellular radio access network traffic: From entropy theory to networking practice," IEEE Commun. Mag., vol. 52, no. 6, pp. 234 240, 2014.

[9] C. Song, Z. Qu, N. Blumm, and A.-L. Barabási, "Limits of predictability in human mobility," Science, vol. 327, no. 5968, pp. 1018-1021, 2010.

[10] J. Manyika, M. Chui, B. Brown, J. Bughin, R. Dobbs, C. Roxburgh, and A. H. Byers, "Big data: The next frontier for innovation, competition, and productivity," McKinsey Global Institute, May 2011.

[11] R. Tandra, S. M. Mishra, and A. Sahai, "What is a spectrum hole and what does it take to recognize one?," Proc. IEEE, vol. 97, no. 5, pp. 824-848, Apr. 2009

[12] J. Yang and H. Zhao, "Enhanced throughput of cognitive radio network$\mathrm{s}$ by imperfect spectrum prediction," IEEE Communications Letters, vol. 19, no. 10, pp. 1738-1741, 2015.

[13] Q. Wu, G. Ding, J. Wang, and Y.-D. Yao, "Spatial-temporal opportunity detection for spectrum-heterogeneous cognitive radio networks: Twodimensional sensing," IEEE Trans. Wireless Commun., vol. 12, no. 2, pp. 516-526, Feb. 2013.
[14] Z. Chen, N. Guo, Z. Hu, and Q. Robert Caiming, "Experimental validation of channel state prediction considering delays in practical cognitive radio," IEEE Trans. Veh. Technol., vol. 60, no. 4, pp. 1314 $1325,2011$.

[15] Z. Wen, T. Luo, W. Xiang, et al, "Autoregressive spectrum hole prediction model for cognitive radio systems," in Proc. IEEE International Conference on Communications Workshops, 19-23 May 2008, Beijing, China, pp. 154-157.

[16] P. Huang, C. Liu, X. Yang, L. Xiao, and J. Chen, "Wireless spectrum occupancy prediction based on partial periodic pattern mining," IEEE Trans. Parallel Distrib. Syst., vol. 25, no. 7, pp. 1925-1934, 2014.

[17] X. Xing, T. Jing, Y. Huo, H. Li, and X. Cheng, "Channel quality prediction based on Bayesian inference in cognitive radio networks," in Proc. IEEE INFOCOM, April 14-19, 2013, Turin, Italy, pp. 1465-1473.

[18] G. P. Villardi and H. Harada, "Computer prediction of primary contour and service areas for unlicensed radio systems operating in TV white space," in Proc. IEEE Wireless Commun. and Networking Conference (WCNC), 07-10 April 2013, Shanghai, China, pp. 4528-4533.

[19] M. Lopez-Benitez and F. Casadevall, "Statistical prediction of spectrum occupancy perception in dynamic spectrum access networks," in Proc. IEEE International Conference on Communications (ICC), 5-9 June 2011, Kyoto, Japan, pp. 1-6.

[20] V. K. Tumuluru, P. Wang, and D. Niyato, "Channel status prediction for cognitive radio networks," Wireless Communications and Mobile Computing, vol. 12, no. 10, pp. 862-874, 2012.

[21] X. Xing, T. Jing, W. Cheng, Y. Huo, and X. Cheng, "Spectrum prediction in cognitive radio networks," IEEE Wireless Commun., vol. 20, no. 2, pp. 90-96, 2013.

[22] Y. Saleem and M. H. Rehmani, "Primary radio user activity models for cognitive radio networks: A survey," Journal of Network and Computer Applications, vol. 43, pp. 1-16, 2014.

[23] M. Hoyhtya, A. Mammela, M. Eskola, M. Matinmikko, J. Kalliovaara, J. Ojaniemi, J. Suutala, R. Ekman, R. Bacchus, D. Roberson, "Spectrum occupancy measurements: A survey and use of interference maps," IEEE Commun. Surveys Tuts., vol. 18, no. 4, pp. 2386-2414, 2016.

[24] Y. Chen and H. S. Oh, "A survey on measurement-based spectrum occupancy modeling for cognitive radios," IEEE Commun. Surveys Tuts., vol. 18, no. 1, pp. 848-859, Firstquarter 2016.

[25] T. Yucek and H. Arslan, "A survey of spectrum sensing algorithms for cognitive radio applications," IEEE Commun. Surveys and Tutorials, vol. 11, no. 1, pp. 116C130, Mar. 2009.

[26] Y. Zeng, Y.-C. Liang, A. T. Hoang, and R. Zhang, "A review on spectrum sensing for cognitive radio: Challenges and solutions," EURASIP Journal on Advances in Signal Processing, vol. 2010, Jan. 2010.

[27] I. F. Akyildiz, B. F. Lo, and R. Balakrishnan, "Cooperative spectrum sensing in cognitive radio networks: A survey," Physical Communication, vol. 4, no. 1, pp. 40-62, Mar. 2011.

[28] E. Axell, G. Leus, E. G. Larsson, and H. V. Poor, "Spectrum sensing for cognitive radio: State-of-the-art and recent advances," IEEE Signal Process. Mag., vol. 29, no.3, pp. 101-116, May 2012.

[29] G. Ding, Q. Wu, Y.-D. Yao, J. Wang, and Y. Chen, "Kernel-based learning for statistical signal processing in cognitive radio networks: Theoretical foundations, example applications, and future directions," IEEE Signal Process. Mag., vol. 30, no. 4, pp. 126-136, 2013.

[30] A. Ali and W. Hamouda, "Advances on spectrum sensing for cognitive radio networks: Theory and applications," IEEE Communications Surveys \& Tutorials, vol. 19, no. 2, pp. 1277-1304, Secondquarter 2017.

[31] M. T. Masonta, M. Mzyece, and N. Ntlatlapa, "Spectrum decision in cognitive radio networks: A survey," IEEE Communications Surveys \& Tutorials, vol. 15, no. 3, pp. 1088-1107, Third Quarter 2013.

[32] L. Gavrilovska, V. Atanasovski, I. Macaluso and L. A. DaSilva, "Learning and reasoning in cognitive radio networks," IEEE Communications Surveys \& Tutorials, vol. 15, no. 4, pp. 1761-1777, Fourth Quarter 2013.

[33] E. Z. Tragos, S. Zeadally, A. G. Fragkiadakis, and V. A. Siris, "Spectrum assignment in cognitive radio networks: A comprehensive survey," IEEE Communications Surveys \& Tutorials, vol. 15, no. 3, pp. 1108-1135, Third Quarter 2013.

[34] E. Ahmed, A. Gani, S. Abolfazli, L. J. Yao, and S. U. Khan, "Channel assignment algorithms in cognitive radio networks: Taxonomy, open issues, and challenges," IEEE Communications Surveys \& Tutorials, vol. 18, no. 1, pp. 795-823, Firstquarter 2016.

[35] W. Liang, S. X. g, and L. Hanzo, "Cooperative overlay spectrum access for cognitive radio networks," to appear in IEEE Communications Surveys \& Tutorials, 2017.

[36] M. Naeem, A. Anpalagan, M. Jaseemuddin, and D. C. Lee, "Resource allocation techniques in cooperative cognitive radio networks," IEEE 
TABLE VII: ACRONYMS

$\begin{array}{ll}\text { 5G } & \text { 5th Generation Mobile Communications } \\ \text { ALE } & \text { Automatic Link Establishment } \\ \text { 4G ALE } & \text { Fourth Generation ALE } \\ \text { APs } & \text { Access Points } \\ \text { AR } & \text { Autoregressive } \\ \text { AR-2 } & \text { A Second Order AR Model } \\ \text { ARIMA } & \text { Autoregressive Integrated Moving Average } \\ \text { ARMA } & \text { Autoregressive Moving Average } \\ \text { BIF } & \text { Bayesian Inference } \\ \text { BP } & \text { Belief Propagation } \\ \text { BSs } & \text { Based Stations } \\ \text { CAV } & \text { Channel Availability Vector } \\ \text { CDF } & \text { Cumulative Distribution Function } \\ \text { CR } & \text { Cognitive Radio } \\ \text { CRNs } & \text { Cognitive Radio Networks } \\ \text { CTMC } & \text { Continuous Time Markov Chain } \\ \text { CTSMC } & \text { Continuous Time Semi Markov Chain } \\ \text { DC } & \text { Duty Cycle } \\ \text { DL } & \text { Dictionary Learning } \\ \text { DSA } & \text { Dynamic Spectrum Access } \\ \text { DTMC } & \text { Discrete Time Markov Chain } \\ \text { ELM } & \text { Extreme Learning Machine } \\ \text { ERNN } & \text { Elman Recurrent Neural Networks } \\ \text { ESN } & \text { Echo State Network } \\ \text { FBNNs } & \text { Feedback Neural Networks } \\ \text { FFNNs } & \text { Feedforward Neural Networks } \\ \text { FPM } & \text { Frequent Pattern Mining } \\ \text { FPM-2D } & \text { FPM in Two Dimension } \\ \text { HBMM } & \text { Hidden Bivariate Markov Model } \\ \text { HF } & \text { High Frequency } \\ \text { LA } & \text { Learning Automata } \\ & \end{array}$

$\begin{array}{ll}\text { LBT } & \text { Listen-Before-Talk } \\ \text { LP } & \text { Linear Prediction } \\ \text { LQA } & \text { Link Quality Analysis } \\ \text { MA } & \text { Moving Average } \\ \text { MAPE } & \text { Mean Absolute Percentage Error } \\ \text { MC } & \text { Matrix Completion } \\ \text { MFNNs } & \text { Multilayer Feedforward Neural Networks } \\ \text { MIMO } & \text { Multiple Input Multiple Output } \\ \text { MLP } & \text { Multilayer Perceptron } \\ \text { MM } & \text { Markov Model } \\ \text { MSE } & \text { Mean Square Error } \\ \text { NMSE } & \text { Normalized Mean Square Error } \\ \text { NS-HMM } & \text { Non Stationary Hidden Markov Model } \\ \text { OP-ELM } & \text { Optimally Pruned Extreme Learning Machine } \\ \text { OSA } & \text { Opportunistic Spectrum Access } \\ \text { PCS } & \text { Personal Communication Service } \\ \text { PFA } & \text { Prediction Fairness Algorithm } \\ \text { PGA } & \text { Prediction Greedy Algorithm } \\ \text { PM } & \text { Pattern Mining } \\ \text { POMDP } & \text { Partially Observable Markov Decision Processes } \\ \text { PPPM } & \text { Partial Periodic Pattern Mining } \\ \text { PSO } & \text { Improved Partial Swarm Optimization } \\ \text { PU } & \text { Primary User } \\ \text { QoS } & \text { Quality Of Service } \\ \text { REM } & \text { Radio Environment Map } \\ \text { RMSE } & \text { Root Mean Square Error } \\ \text { RSS } & \text { Radio Spectrum State } \\ \text { SLFNs } & \text { Single Hidden Layer Neural Networks } \\ \text { SNG } & \text { Static Neighbor Graph } \\ \text { SVM } & \text { Support Vector Machine } \\ \text { SVR } & \text { Support Vector Regression } \\ & \end{array}$

Communications Surveys \& Tutorials, vol. 16, no. 2, pp. 729-744, Second Quarter 2014.

[37] Y. Xu, X. Zhao, and Y. C. Liang, "Robust power control and beamforming in cognitive radio networks: A survey," IEEE Communications Surveys \& Tutorials, vol. 17, no. 4, pp. 1834-1857, Fourthquarter 2015.

[38] A. Ahmad, S. Ahmad, M. H. Rehmani, and N. U. Hassan, "A survey on radio resource allocation in cognitive radio sensor networks," IEEE Communications Surveys \& Tutorials, vol. 17, no. 2, pp. 888-917, Secondquarter 2015

[39] G. I. Tsiropoulos, O. A. Dobre, M. H. Ahmed and K. E. Baddour, "Radio resource allocation techniques for efficient spectrum access in cognitive radio networks," IEEE Communications Surveys \& Tutorials, vol. 18, no. 1, pp. 824-847, Firstquarter 2016.

[40] S. H. R. Bukhari, M. H. Rehmani, and S. Siraj, "A survey of channel bonding for wireless networks and guidelines of channel bonding for futuristic cognitive radio sensor networks," IEEE Communications Surveys \& Tutorials, vol. 18, no. 2, pp. 924-948, Secondquarter 2016.

[41] M. El Tanab and W. Hamouda, "Resource allocation for underlay cognitive radio networks: A survey," IEEE Communications Surveys \& Tutorials, vol. 19, no. 2, pp. 1249-1276, Secondquarter 2017.

[42] G. Baldini, T. Sturman, A. R. Biswas, R. Leschhorn, G. Godor and M. Street, "Security aspects in software defined radio and cognitive radio networks: A survey and a way ahead," IEEE Communications Surveys \& Tutorials, vol. 14, no. 2, pp. 355-379, Second Quarter 2012.

[43] A. Attar, H. Tang, A. V. Vasilakos, and F. R. Yu, "A survey of security challenges in cognitive radio networks: Solutions and future research directions," Proc. IEEE, vol. 100, no. 12, pp. 3172-3186, Dec. 2012.

[44] R. P. Helena, J. B. Mercedes, and G. Carles, "Review of robust cooperative spectrum sesnsing techniques for cognitive radio networks," Wireless Pers. Commun., vol. 67, no. 2, pp. 175-198, Nov. 2012.

[45] A. G. Fragkiadakis, E. Z. Tragos, and I. G. Askoxylakis, "A survey on security threats and detection techniques in cognitive radio networks," IEEE Communications Surveys \& Tutorials, vol. 15, no. 1, pp. 428-445, First Quarter 2013.

[46] L. Zhang, G. Ding, Q. Wu, Y. Zou, Z. Han and J. Wang, "Byzantine attack and defense in cognitive radio networks: A survey," IEEE Communications Surveys \& Tutorials, vol. 17, no. 3, pp. 1342-1363, thirdquarter 2015.

[47] R. K. Sharma and D. B. Rawat, "Advances on security threats and countermeasures for cognitive radio networks: A survey," IEEE
Communications Surveys \& Tutorials, vol. 17, no. 2, pp. 1023-1043, Secondquarter 2015.

[48] M. Grissa, B. Hamdaoui, and A. A. Yavuz, "Location privacy in $\operatorname{cog}$ nitive radio networks: A survey," to appear in IEEE Communications Surveys \& Tutorials, 2017.

[49] A. De Domenico, E. Calvanese Strinati, and M. G. Di Benedetto, "A survey on MAC strategies for cognitive radio networks," IEEE Communications Surveys \& Tutorials, vol. 14, no. 1, pp. 21-44, First Quarter 2012.

[50] L. Gavrilovska, D. Denkovski, V. Rakovic, and M. Angjelichinoski, "Medium access control protocols in cognitive radio networks: Overview and general classification," IEEE Communications Surveys \& Tutorials, vol. 16, no. 4, pp. 2092-2124, Fourthquarter 2014.

[51] M. Youssef, M. Ibrahim, M. Abdelatif, L. Chen and A. V. Vasilakos, "Routing metrics of cognitive radio networks: A survey," IEEE Communications Surveys \& Tutorials, vol. 16, no. 1, pp. 92-109, First Quarter 2014.

[52] A. Naeem, M. H. Rehmani, Y. Saleem, I. Rashid, N. Crespi, "Network coding in cognitive radio networks: A comprehensive survey," to appear in IEEE Communications Surveys \& Tutorials, 2017.

[53] X. Chen, H. H. Chen, and W. Meng, "Cooperative communications for cognitive radio networks- From theory to applications," IEEE Communications Surveys \& Tutorials, vol. 16, no. 3, pp. 1180-1192, Third Quarter 2014.

[54] X. Huang, T. Han, and N. Ansari, "On green-energy-powered cognitive radio networks," IEEE Communications Surveys \& Tutorials, vol. 17, no. 2, pp. 827-842, Secondquarter 2015.

[55] H. Ding, Y. Fang, X. Huang, M. Pan, P. Li, and S. Glisic, "Cognitive capacity harvesting networks: Architectural evolution towards future cognitive radio networks," to appear in IEEE Communications Surveys \& Tutorials, 2017.

[56] A. A. Khan, M. H. Rehmani, and M. Reisslein, "Cognitive radio for smart grids: Survey of architectures, spectrum sensing mechanisms, and networking protocols," IEEE Communications Surveys \& Tutorials, vol. 18, no. 1, pp. 860-898, Firstquarter 2016.

[57] Spectrum Policy Task Force, "Report of the Spectrum Efficiency Working Group," Federal Communications Commission, Washington, D.C., USA, Tech. Rep., November 2002.

[58] A. Azarfar, J. F. Frigon, and B. Sanso, "Improving the reliability of wireless networks using cognitive radios," IEEE Communications Surveys \& Tutorials, vol. 14, no. 2, pp. 338-354, Second Quarter 2012. 
[59] F. Sanders, "Broadband spectrum surveys in Denver, CO, San Diego, CA, and Los Angeles, CA: Methodology, analysis, and comparative results," in Proc. IEEE International Symposium on Electromagnetic Compatibility, August 24-28, 1998, Denver, Colorado, USA, vol. 2, pp. 988-993.

[60] F. H. Sanders, B. J. Ramsey, and V. S. Lawrence, "Broadband spectrum survey at San Diego, California," NASA, no. 19980005224, 1996.

[61] M. Biggs, A. Henley, and T. Clarkson, "Occupancy analysis of the 2.4 GHz ISM band,' IEE Proc. Communications, vol. 151, no. 5, pp. 481-488, Oct 2004.

[62] M. McHenry and D. McCloskey, "Spectrum occupancy measurements: Chicago, Illinois, November 16-18, 2005." Shared Spectrum Company, Tech. Rep., 2005.

[63] A. Petrin and P. G. Steffes, "Analysis and comparison of spectrum measurements performed in urban and rural areas to determine the total amount of spectrum usage," in Proc. International Symposium on Advanced Radio Technologies (ISART), 1-3 Mar. 2005, Boulder, CO, USA, pp. 9-12.

[64] A. J. Petrin, "Maximizing the utility of radio spectrum: Broadband spectrum measurements and occupancy model for use by cognitive radio," Ph.D. Dissertation, Georgia Institute of Technology 2005.

[65] M. A. McHenry, P. A. Tenhula, D. McCloskey, D. A. Roberson, and C. S. Hood, "Chicago spectrum occupancy measurements \& analysis and a long-term studies proposal," in Proc. 1st International Workshop on Technology and Policy for Accessing Spectrum, 5 Aug. 2006, Boston, MA, USA, pp. 1-5.

[66] D. A. Roberson, C. S. Hood, J. L. LoCicero, and J. T. MacDonald "Spectral occupancy and interference studies in support of cognitive radio technology deployment," in Proc. 1st IEEE Workshop on Networking Technologies for Software Defined Radio Networks, Reston, VA, USA, 2006, pp. 26-35.

[67] V. Blaschke, H. Jäkel, T. Renk, C. Kloeck, and F. K. Jondral, "Occupation measurements supporting dynamic spectrum allocation for cognitive radio design," in Proc. 2nd International Conference on Cognitive Radio Oriented Wireless Networks and Communications (CrownCom), July 31-Aug. 3 2007, Orlando, USA, pp. 50-57.

[68] R. I. Chiang, G. B. Rowe, and K. W. Sowerby, "A quantitative analysis of spectral occupancy measurements for cognitive radio," in Proc. IEEE 65th Vehicular Technology Conference-Spring, 22-25 Apr. 2007, Dublin, Ireland, pp. 3016-3020

[69] T. Erpek, M. Lofquist, and K. Patton, "Spectrum occupancy measurements loring commerce centre limestone, Maine September 18-20, 2007," The Shared Spectrum Company, 2007.

[70] T. Erpek, K. Steadman, and D. Jones, "Dublin ireland spectrum occupancy measurements collected on April 16-18, 2007," The Shared Spectrum Company, 2007.

[71] S. D. Jones, E. Jung, X. Liu, N. Merheb, and I.-J. Wang, "Characterization of spectrum activities in the US public safety band for opportunistic spectrum access," in Proc. 2nd IEEE International Symposium on New Frontiers in Dynamic Spectrum Access Networks, 17-20 Apr. 2007 Dublin, Ireland, pp. 137-146.

[72] M. Wellens, J. Wu, and P. Mahonen, "Evaluation of spectrum occupancy in indoor and outdoor scenario in the context of cognitive radio," in Proc. 2nd International Conference on Cognitive Radio Oriented Wireless Networks and Communications, July 31-Aug. 3 2007, Orlando, USA, pp. 420-427.

[73] R. B. Bacchus, A. J. Fertner, C. S. Hood, and D. A. Roberson, "Long-term, wide-band spectral monitoring in support of dynamic spectrum access networks at the iit spectrum observatory," in Proc. 3rd IEEE Symposium on New Frontiers in Dynamic Spectrum Access Networks(DySPAN), 14-18 Oct. 2008, Chicago, USA, pp. 1-10.

[74] M. H. Islam, C. L. Koh, S. W. Oh, X. Qing, Y. Y. Lai, C. Wang, Y.-C. Liang, B. E. Toh, F. Chin, and G. L. Tan, "Spectrum survey in singapore: Occupancy measurements and analyses," in Proc. 3rd International Conference on Cognitive Radio Oriented Wireless Networks and Communications, 15-17 May, 2008, Singore, pp. 1-7.

[75] M. Lopez-Benitez, A. Umbert, and F. Casadevall, "Evaluation of spectrum occupancy in Spain for cognitive radio applications," in Proc. IEEE 69th Vehicular Technology Conference, 26-29 Apr. 2009, Barcelona, Spain, pp. 1-5.

[76] K. A. Qaraqe, H. Celebi, A. Gorcin, A. El-Saigh, H. Arslan, and M.-S. Alouini, "Empirical results for wideband multidimensional spectrum usage," in Proc. IEEE 20th International Symposium on Personal, Indoor and Mobile Radio Communications, 13-16 Sep. 2009, Tokyo, Japan, pp. 1262-1266.

[77] R. Bacchus, T. Taher, K. Zdunek, and D. Roberson, "Spectrum utilization study in support of dynamic spectrum access for public safety," in
Proc. IEEE Symposium on New Frontiers in Dynamic Spectrum Access Networks, 06-09 Apr. 2010, Singapore, pp. 1-11.

[78] R. Schiphorst and C. H. Slump, "Evaluation of spectrum occupancy in amsterdam using mobile monitoring vehicles," in Proc. IEEE 71st Vehicular Technology Conference, 16-19 May 2010, Taipei, Taiwan, pp. 1-5.

[79] V. Valenta, R. Marsalek, G. Baudoin, M. Villegas, M. Suarez, and F. Robert, "Survey on spectrum utilization in europe: Measurements, analyses and observations," in Proc. 5th International Conference on Cognitive Radio Oriented Wireless Networks \& Communications, 9-11 Jun. 2010, Cannes, France, pp. 1-5.

[80] S. Contreras, G. Villardi, R. Funada, and H. Harada, "An investigation into the spectrum occupancy in japan in the context of tv white space systems," in Proc. Sixth International ICST Conference on Cognitive Radio Oriented Wireless Networks and Communications, 1-3 Jun. 2011, Osaka, Japan, pp. 341-345.

[81] T. Harrold, R. Cepeda, and M. Beach, "Long-term measurements of spectrum occupancy characteristics," in Proc. IEEE Symposium on New Frontiers in Dynamic Spectrum Access Networks (DySPAN), 3-6 May 2011, Aachen, Germany, pp. 83-89.

[82] T. M. Taher, R. B. Bacchus, K. J. Zdunek, and D. A. Roberson, "Longterm spectral occupancy findings in chicago," in Proc. IEEE Symposium on New Frontiers in Dynamic Spectrum Access Networks (DySPAN), 3-6 May 2011, Aachen, Germany, pp. 100-107.

[83] J. Kokkoniemi and J. Lehtomaki, "Spectrum occupancy measurements and analysis methods on the $2.45 \mathrm{GHz}$ ISM band," in Proc. 7th International ICST Conference on Cognitive Radio Oriented Wireless Networks and Communications (CROWNCOM), 18-20 Jun. 2012, Stockholm, Sweden, pp. 285-290.

[84] R. Urban, T. Korinek, and P. Pechac, "Broadband spectrum survey measurements for cognitive radio applications," Radioengineering, vol. 21, no. 4, pp. 1101-1109, 2012.

[85] L. F. Pedraza, F. Forero, and I. Paez, "Metropolitan spectrum survey in Bogota Colombia," in Proc. 27th International Conference on Advanced Information Networking and Applications Workshops (WAINA), 25-28 Mar. 2013, Barcelona, Spain, pp. 548-553.

[86] K. Chen, J. Min, X. Han, X. Yan, Y. Duan, L. Zhang, and Z. Feng, "Spectrum survey for TV band in Beijing," in Proc. 21st International Conference on Telecommunications (ICT), 5-7 May 2014, Lisbon, Portugal, pp. 267-271.

[87] O. Holland, H. Kokkinen, S. Wong, V. Friderikos, A. Raman, M. Dohler, and M. Lema, "Changing availability of TV white space in the UK," IEE Electronics Letters, vol. 52, no. 15, pp. 1349-1351, 2016.

[88] B. Bochow, O. Holland, K. Katzis, "Spectrum sensing infrastructure support for IEEE 1900.6b sensing-assisted spectrum databases," in Proc. IEEE Conference on Standards for Communications \& Networking (CSCN'16), 31 Oct.-2 Nov. 2016, Berlin, Germany, pp. 1-6.

[89] M. López-Ben ítez, "Spectrum usage models for the analysis, design and simulation of cognitive radio networks," Cognitive radio and its application for next generation cellular and wireless networks, Springer Netherlands, pp. 27-73, 2012.

[90] C. Ghosh, S. Pagadarai, D. P. Agrawal, and A. M. Wyglinski, "A framework for statistical wireless spectrum occupancy modeling," IEEE Trans. Wireless Commun., vol. 9, no. 1, pp. 38-44, 2010.

[91] M. Wellens, J. RiihijäRvi, and P. MäHöNen, "Empirical time and frequency domain models of spectrum use," Physical Communication, vol. 2, no. 1, pp. 10-32, 2009.

[92] P. F. Marshall, "Closed-form analysis of spectrum characteristics for cognitive radio performance analysis," in Proc. 3rd IEEE Symposium on New Frontiers in Dynamic Spectrum Access Networks (DySpan), 14-17 Oct. 2008, Chicago, USA, pp. 1-12.

[93] A. Gorcin, H. Celebi, K. A. Qaraqe, and H. Arslan, "An autoregressive approach for spectrum occupancy modeling and prediction based on synchronous measurements," in Proc. IEEE 22nd International Symposium on Personal Indoor and Mobile Radio Communications (PIMRC), 11-14 Sep. 2011, Toronto, Canada, pp. 705-709.

[94] S. Ki Won, K. Seong-Lyun, and J. Zander, "Temporal spectrum sharing based on primary user activity prediction," IEEE Trans. Wireless Commun., vol. 9, no. 12, pp. 3848-3855, 2010.

[95] I. A. Akbar and W. H. Tranter, "Dynamic spectrum allocation in cognitive radio using hidden markov models: Poisson distributed case," in Proc. IEEE SoutheastCon, 2007., Richmond, VA, pp. 196-201.

[96] A. Papoulis and S. U. Pillai, Probability, random variables, and stochastic processes. Tata McGraw-Hill Education, 2002.

[97] P. Kumaraswamy, "A generalized probability density function for 
double-bounded random processes," Journal of Hydrology, vol. 46, no. 1 , pp. 79-88, 1980.

[98] A. U. Rehman, V. A. Thomas, L. L. Yang, and L. Hanzo, "Performance of cognitive selective-repeat hybrid automatic repeat request," IEEE Access, vol. 4, pp. 9828-9846, 2016.

[99] A. U. Rehman, C. Dong, L. L. Yang, and L. Hanzo, "Performance of cognitive stop-and-wait hybrid automatic repeat request in the face of imperfect sensing," IEEE Access, vol. 4, pp. 5489-5508, 2016.

[100] Z. Liang and D. Zhao, "Quality of service performance of a cognitive radio sensor network," in Proc. IEEE International Conference on Communications (ICC), 23-27 May 2010, Cape Town, South Africa, pp. 1-5.

[101] L. Yang, L. Cao, and H. Zheng, "Proactive channel access in dynamic spectrum networks," Physical communication, vol. 1, no. 2, pp. $103-$ $111,2008$.

[102] M. Hamid, A. Mohammed, and Z. Yang, "On spectrum sharing and dynamic spectrum allocation: MAC layer spectrum sensing in cognitive radio networks," in Proc. International Conference on Communications and Mobile Computing (CMC), 12-14 Apr. 2010, Shenzhen, China, vol. 2, pp. 183-187.

[103] C. Guo, T. Peng, Y. Qi, and W. Wang, "Adaptive channel searching scheme for cooperative spectrum sensing in cognitive radio networks," in Proc. IEEE Wireless Communications and Networking Conference (WCNC), 5-8 Apr. 2009, Budapest, Hungary, pp. 1-6.

[104] S. Geirhofer, L. Tong, and B. M. Sadler, "Dynamic spectrum access in WLAN channels: Empirical model and its stochastic analysis," in Proc. 1st international workshop on Technology and policy for accessing spectrum, 5 Aug. 2006, Boston, MA, USA pp. 1-10.

[105] — "Cognitive radios for dynamic spectrum access-dynamic spectrum access in the time domain: Modeling and exploiting white space," IEEE Commun. Mag., vol. 45, no. 5, pp. 66-72, 2007.

[106] L. Stabellini, "Quantifying and modeling spectrum opportunities in a real wireless environment," in Proc. IEEE Wireless Communications and Networking Conference (WCNC), 18-21 Apr. 2010, Sydney Australia, pp. 1-6.

[107] M. Abramowitz and I. A. Stegun, Handbook of Mathematical Functions: With Formulas, Graphs, and Mathematical Tables. Courier Dover Publications, 1972.

[108] S. Geirhofer, L. Tong, and B. M. Sadler, "A measurement-based model for dynamic spectrum access in WLAN channels," in Proc. IEEE Military Communications Conference (MILCOM), 23-25 Oct. 2006, Washington, DC, USA, pp. 1-7.

[109] M. Wellens, J. Riihijarvi, and P. Mahonen, "Modelling primary system activity in dynamic spectrum access networks by aggregated on/offprocesses," in Proc. 6th Annual IEEE Communications Society Conference on Sensor, Mesh and Ad Hoc Communications and Networks Workshops, 22-26 Jun. 2009, Rome, Italy, pp. 1-6.

[110] Q. Zhao and K. Liu, "Detecting, tracking, and exploiting spectrum opportunities in unslotted primary systems," in Proc. IEEE Radio and Wireless Symposium, 22-24 Jan. 2008, Orlando, Florida, USA, pp. 491494.

[111] L. Melián-Gutiérrez, S. Zazo, J. L. Blanco-Murillo, I. Pérezálvarez, A. Garcła-Rodrłguez, and B. Prez-Dłaz, "HF spectrum activity prediction model based on HMM for cognitive radio applications," Physical Communication, vol. 9, pp. 199-211, 2013.

[112] D. Willkomm, S. Machiraju, J. Bolot, and A. Wolisz, "Primary users in cellular networks: A large-scale measurement study," in Proc. 3rd IEEE symposium on New frontiers in dynamic spectrum access networks(DySPAN), 14-17 Oct. 2008, Chicago, USA, pp. 1-11.

[113] X. Hong, C.-X. Wang, H.-H. Chen, and Y. Zhang, "Secondary spectrum access networks," IEEE Veh. Technol. Mag., vol. 4, no. 2, pp. 36-43, 2009.

[114] Z. Chen, C.-X. Wang, X. Hong, J. Thompson, S. A. Vorobyov, and $\mathrm{X}$. Ge, "Interference modeling for cognitive radio networks with power or contention control," in Proc. IEEE Wireless Communications and Networking Conference (WCNC), 18-21 Apr. 2010, Sydney, Australia, pp. 1-6.

[115] Y. Zhao, L. Morales, J. Gaeddert, K. K. Bae, J.-S. Um, and J. H. Reed, "Applying radio environment maps to cognitive wireless regional area networks," in Proc. IEEE Symposium on New Frontiers in Dynamic Spectrum Access Networks (DySPAN), 14-17 Oct. 2008, Chicago, USA, pp. $115-118$.

[116] Y. Zhao, B. Le, and J. H. Reed, "Network support the radio environment map," Cognitive radio technology, pp. 325-366, 2006.

[117] J. J. Lehtomaki, R. Vuohtoniemi, and K. Umebayashi, "On the measurement of duty cycle and channel occupancy rate," IEEE J. Sel. Areas Commun., vol. 31 , no. 11 , pp. 2555-2565, 2013.
[118] X. Chen, H. Zhang, A. MacKenzie, and M. Matinmikko, "Predicting spectrum occupancies using a non-stationary hidden markov model," IEEE Commun. Lett., vol. 3, no. 4, pp. 333-336, Aug. 2014.

[119] S. Yin, D. Chen, Q. Zhang, M. Liu, and S. Li, "Mining spectrum usage data: A large-scale spectrum measurement study," IEEE Trans. Mobile Comput., vol. 11, no. 6, pp. 1033-1046, 2012.

[120] A. Goldsmith and I. Maric, "Capacity of cognitive radio networks," Principles of Cognitive Radio Networks, Cambridge University Press, ISBN:9781139854276. Nov. 2012.

[121] F. Paisana, N. Marchetti, and L. A. DaSilva, "Radar, TV and cellular bands: Which spectrum access techniques for which bands?" IEEE Communications Surveys \& Tutorials, vol. 16, no. 3, pp. 1193-1220, Third Quarter 2014.

[122] F. Akhtar, M. H. Rehmani, and M. Reisslein, "White space: Definitional perspectives and their role in exploiting spectrum opportunities,' Telecommunications Policy, vol. 40, no. 4, pp. 319-331, 2016.

[123] M. P. Olivieri, G. Barnett, A. Lackpour, A. Davis, and P. Ngo, "A scalable dynamic spectrum allocation system with interference mitigation for teams of spectrally agile software defined radios," in Proc. IEEE Symposium on New. Frontiers in Dynamic Spectrum Access Networks (DySPAN), 8-11 Nov. 2005, Baltimore, Maryland, USA, pp. 170-179.

[124] M. Wellens, J. Riihijarvi, and P. Mahonen, "Evaluation of spectrum occupancy using approximate and multiscale entropy metrics," in Proc. 5th IEEE Annual Communications Society Conference on Sensor, Mesh and Ad Hoc Communications and Networks Workshops, 16-20 Jun. 2008, California, USA, pp. 1-8.

[125] J. Riihijarvi, P. Mahonen, and M. Wellens, "Metrics for characterizing complexity of network traffic," in Proc. International Conference on Telecommunications, 16-19 Jun. 2008, St. Petersburg, Russia, pp. 1-6.

[126] J. Riihijarvi, M. Wellens, and P. Mahonen, "Measuring complexity and predictability in networks with multiscale entropy analysis," in Proc. IEEE INFOCOM, 19-25 Apr. 2009, Rio de Janeiro, Brazil, pp. 11071115 .

[127] G. Ding, J. Wang, Q. Wu, et al., "On the limits of predictability in real-world radio spectrum state dynamics: From entropy theory to 5G spectrum sharing," IEEE Commun. Mag., vol. 53, no. 7, pp. 178-183, July 2015.

[128] J. Su and W. Wu, "Wireless spectrum prediction model based on time series analysis method," in Proc. ACM workshop on Cognitive Radio Networks, 20-25 Sep. 2009, Beijing, China, pp. 61-66.

[129] Z. Lin, X. Jiang, L. Huang, and Y. Yao, "A energy prediction based spectrum sensing approach for cognitive radio networks," in Proc. 5th International Conference on Wireless Communications, Networking and Mobile Computing, 24-26 Sep. 2009, Beijing, China, pp. 1-4.

[130] S. Yarkan and H. Arslan, "Binary time series approach to spectrum prediction for cognitive radio," in Proc. IEEE 66th Vehicular Technology Conference, Sep. 30-Oct. 3 2007, Baltimore, MD, USA, pp. 1563-1567.

[131] L. Hanzo, C. Somerville, and J. Woodard, Voice and Audio Compression for Wireless Communications. John Wiley \& Sons, 2008.

[132] L. Hanzo, P. Cherriman, and J. Streit, Video Compression and Communications: From Basics to H. 261, H. 263, H. 264, MPEG4 for DVB and HSDPA-Style Adaptive Turbo-Transceivers. John Wiley \& Sons, 2007.

[133] J. G. Proakis and D. K Manolakis, Digital Signal Processing: Principles, Algorithms and Applications, Pearson, 2006 (4th edtion).

[134] P.-Y. Wu and A. Duel-Hallen, "Multiuser detectors with disjoint Kalman channel estimators for synchronous CDMA mobile radio channels," IEEE Trans. Commun., vol. 48, no. 5, pp. 752-756, 2000.

[135] P. Sharma and K. Chandra, "Prediction of state transitions in Rayleigh fading channels," IEEE Trans. Veh. Technol., vol. 56, no. 2, pp. 416$425,2007$.

[136] R. Matias, A. M. Carvalho, V. A. Teodoro, D. Tes, and L. B. Araujo, "Performance comparison of forecasting models applied to lan/man traffic prediction," in Proc. IEEE Workshop on Local \& Metropolitan Area Networks (LANMAN), 13-14 Oct. 2011, Chapel Hill, NC, USA, pp. 1-6.

[137] J. Su and W. Wu, "Wireless spectrum prediction model based on time series analysis method," in Proc. ACM workshop on Cognitive radio networks, 20-25 Sep. 2009, Beijing, China, pp. 61-66.

[138] A. A. Tabassam, M. U. Suleman, S. Khan, and S. H. R. Tirmazi, "Spectrum estimation and spectrum hole opportunities prediction for cognitive radios using higher-order statistics," in Proc. Wireless Advanced (WiAd), 20-22 June 2011, London, United Kingdom, pp. 213217. 
[139] C. Stolojescu-Crisan, "Data mining based wireless network traffic forecasting," in Proc. 10th International Symposium on Electronics and Telecommunications (ISETC), 15-16 Nov. 2012, Timisoara, Romania, pp. $115-118$

[140] C. Ghosh, C. Cordeiro, D. P. Agrawal, and M. B. Rao, "Markov chain existence and hidden Markov models in spectrum sensing," in Proc. IEEE International Conference on Pervasive Computing and Communications, 9-13 Mar. 2009, Galveston, Texas, pp. 1-6.

[141] L. Rabiner, "A tutorial on hidden markov models and selected applications in speech recognition," Proceedings of the IEEE, vol. 77, no. 2, pp. 257-286, 1989.

[142] T. Black, B. Kerans, and A. Kerans, "Implementation of hidden Markov model spectrum prediction algorithm," in Proc. International Symposium on Communications and Information Technologies (ISCIT), 02-05 Oct. 2012, Gold Coast, Australia, pp. 280-283.

[143] E. Chatziantoniou, B. Allen, and V. Velisavljevic, "An HMM-based spectrum occupancy predictor for energy efficient cognitive radio," in Proc. IEEE 24th International Symposium on Personal Indoor and Mobile Radio Communications (PIMRC), 8-11 Sep. 2013, London UK, pp. 601-605.

[144] N. Thao, B. L. Mark, and Y. Ephraim, "Spectrum sensing using a hidden bivariate Markov model," IEEE Trans. Wireless Commun., vol. 12, no. 9, pp. 4582-4591, 2013.

[145] L. E. Baum and T. Petrie, "Statistical inference for probabilistic functions of finite state Markov chains," Ann. Math. Statist., vol. 37, no. 6, pp. 1554-1563, 1966.

[146] Z. Chen and R. C. Qiu, "Prediction of channel state for cognitive radio using higher-order hidden Markov model," in Proc. IEEE SoutheastCon, 18-21 Mar. 2010, Charlotte, North Carolina, USA, pp. 276-282.

[147] H. A. Engelbrecht, "Efficient decoding of high-order hidden Markov models," Ph.D. dissertation, Stellenbosch University, 2007.

[148] J. A. du Preez, "Efficient training of high-order hidden Markov models using first-order representations," Computer speech \& language, vol. 12 , no. 1 , pp. 23-39, 1998.

[149] Z. Chen, "A study and implementation of speech recognition system for mobile communication terminals," Master's thesis, Hangzhou Dianzi University, 2003.

[150] G. E. Monahan, "State of the art - A survey of partially observable Markov decision processes: Theory, models, and algorithms," Management Science, vol. 28, no. 1, pp. 1-16, 1982.

[151] L. P. Kaelbling, M. L. Littman, and A. R. Cassandra, "Planning and acting in partially observable stochastic domains," Artificial intelligence, vol. 101, no. 1, pp. 99-134, 1998.

[152] H. Fang, L. Xu, and C. Huang, "Dynamic opportunistic spectrum access of multi-channel multi-radio based on game theory in wireless cognitive network," in Proc. IEEE Ninth International Conference on Mobile Ad-hoc and Sensor Networks (MSN), 11-13 Dec. 2013, Dalian, China, pp. 127-132.

[153] Q. Zhao, L. Tong, A. Swami, and Y. Chen, "Decentralized cognitive mac for opportunistic spectrum access in ad hoc networks: A POMDP framework," IEEE J. Sel. Areas Commun., vol. 25, no. 3, pp. 589-600, 2007.

[154] A. T. Hoang, Y.-C. Liang, D. T. C. Wong, Y. Zeng, and R. Zhang, "Opportunistic spectrum access for energy-constrained cognitive radios," IEEE Trans. Wireless Commun., vol. 8, no. 3, pp. 1206-1211, 2009.

[155] C. Clancy, J. Hecker, E. Stuntebeck, and T. O’Shea, "Applications of machine learning to cognitive radio networks," IEEE Wireless Commun., vol. 14, no. 4, pp. 47-52, 2007.

[156] Q. Zhao and S. B. M., "A survey of dynamic spectrum access," IEEE Signal Process. Mag., vol. 24, no. 3, pp. 79-89, 2007.

[157] M. Golestanian, S. Iranmanesh, R. Ghazizadeh, and M. Azimi, "A learning automata based spectrum prediction technique for cognitive radio networks," Science and Education, vol. 2, no. 3, pp. 93-97, 2014.

[158] L. Yang, N. Lv, and Z. X. Xu, "Spectrum prediction for cognitive radio system based on optimally pruned extreme learning machine," Applied Mechanics and Materials, vol. 536, pp. 430-436, 2014.

[159] Y. Miche, A. Sorjamaa, P. Bas, O. Simula, C. Jutten, and A. Lendasse, "OP-ELM: optimally pruned extreme learning machine," IEEE Trans. Neural Netw., vol. 21, no. 1, pp. 158-162, 2010.

[160] H. Li, X. Xu, B. Wu, and X. Chen, "Multilayer feedforward neural network based efficient spectrum occupancy prediction scheme for cognitive radio system," Journal of Computational Information Systems, vol. 10, no. 10, pp. 4017-4028, 2014.

[161] S. Bai, X. Zhou, and F. Xu, “'Soft decision' spectrum prediction based on back-propagation neural networks," in Proc. Internation- al Conference on Computing, Management and Telecommunications (ComManTel), 27-29 April 2014, Da Nang, Vietnam, pp. 128-133.

[162] L. Yin, S. Yin, W. Hong, and S. Li, "Spectrum behavior learning in cognitive radio based on artificial neural network," in Proc. Military Communications Conference, 07-10 Nov. 2011, Baltimore, MD, USA, pp. 25-30.

[163] V. K. Tumuluru, W. Ping, and D. Niyato, "A neural network based spectrum prediction scheme for cognitive radio," in Proc. IEEE International Conference on Communications (ICC), 23-27 May 2010, Cape Town, South Africa, pp. 1-5.

[164] J. Zhao, M. Wang, and J. Yuan, "Based on neural network spectrum prediction of cognitive radio," in Proc. International Conference on Electronics, Communications and Control (ICECC), 09-11 Sep. 2011, Ningbo, China, pp. 762-765.

[165] O. Winston, A. Thomas, and W. OkelloOdongo, "Optimizing neural network for tv idle channel prediction in cognitive radio using particle swarm optimization," in Proc. 5th International Conference on Computational Intelligence, Communication Systems and Networks (CICSyN), 5-7 Jun. 2013, Madrid, Spain, pp. 25-29.

[166] O. Winston, A. Thomas, O. Patrick, and O. William, "PSO of neural networks to predict busy times of cellular traffic for assignment to TV idle channels by cognitive radio," in Proc. European Modelling Symposium (EMS), 20-22 Nov. 2013, Manchester, UK, pp. 48-52.

[167] N. Baldo, B. R. Tamma, B. Manoj, R. Rao, and M. Zorzi, "A neural network based cognitive controller for dynamic channel selection," in Proc. IEEE International Conference on Communications (ICC), 14-18 June 2009, Dresden, Germany, pp. 1-5.

[168] N. Baldo and M. Zorzi, "Learning and adaptation in cognitive radios using neural networks," in Proc. 5th IEEE Consumer Communications and Networking Conference, 10-12 Jan. 2008, Las Vegas, Nevada, pp. 998-1003.

[169] S. Haykin and N. Network, "Neural networks: A comprehensive foundation," Neural Networks, vol. 2, no. 2004, 2004.

[170] I. Christian, et al., "Spectrum mobility in cognitive radio networks," IEEE Communications Magazine, vol. 50, no. 6, pp. 114-121, Jun. 2012.

[171] K. Kumar, A. Prakash, and R. Tripathi, "Spectrum handoff in cognitive radio networks: A classification and comprehensive survey," Journal of Network and Computer Applications, vol. 61, pp. 161-188, 2016.

[172] Y.-S. Chen, et al., "A cross-layer protocol of spectrum mobility and handover in cognitive LTE networks," Simulation modelling practice and theory, vol. 19, no. 8, pp. 1723-1744, 2011.

[173] W. Y. Lee and I. F. Akyildiz, "Spectrum-aware mobility management in cognitive radio cellular networks," IEEE Transactions on Mobile Computing, vol. 11, no. 4, pp. 529-542, Apr. 2012.

[174] X. Huang, J. Wu, W. Li, Z. Zhang, F. Zhu and M. Wu, "Historical spectrum sensing data mining for cognitive radio enabled vehicular ad-hoc networks," IEEE Transactions on Dependable and Secure Computing, vol. 13, no. 1, pp. 59-70, Jan. 2016.

[175] P. Thakur, et al., "Spectrum mobility in cognitive radio network using spectrum prediction and monitoring techniques," Physical Communication, vol. 24, pp. 1-8, 2017.

[176] H. Zhou, N. Cheng, Q. Yu, X. Sherman Shen, D. Shan and F. Bai, "Toward multi-radio vehicular data piping for dynamic DSRC/TVWS spectrum sharing," IEEE Journal on Selected Areas in Communications, vol. 34, no. 10, pp. 2575-2588, Oct. 2016.

[177] G.-B. Huang, Q.-Y. Zhu, and C.-K. Siew, "Extreme learning machine: theory and applications," Neurocomputing, vol. 70, no. 1, pp. 489-501, 2006.

[178] M. I. Taj and M. Akil, "Cognitive radio spectrum evolution prediction using artificial neural networks based multivariate time series modelling," in Proc. 11th European Wireless Conference on Sustainable Wireless Technologies (European Wireless) 2011, Vienna, Austria, pp. 1-6.

[179] A. Katidiotis, K. Tsagkaris, and P. Demestichas, "Performance evaluation of artificial neural network-based learning schemes for cognitive radio systems," Computers \& Electrical Engineering, vol. 36, no. 3, pp. 518-535, 2010.

[180] G. Dematos, M. S. Boyd, B. Kermanshahi, N. Kohzadi, and I. Kaastra "Feedforward versus recurrent neural networks for forecasting monthly Japanese Yen exchange rates," Financial Engineering and the Japanese Markets, vol. 3, no. 1, pp. 59-75, 1996.

[181] D. Verstraeten, B. Schrauwen, M. dHaene, and D. Stroobandt, "An experimental unification of reservoir computing methods," Neural Networks, vol. 20, no. 3, pp. 391-403, 2007.

[182] H. Jaeger, "The 'echo state' approach to analysing and training recurrent neural networks-with an erratum note," Bonn, Germany: German 
National Research Center for Information Technology GMD Technical Report, vol. 148, p. 34, 2001.

[183] L. Yang, X. Liang, T. Ma, and K. Liu, "Spectrum prediction based on echo state network and its improved form," in Proc. 5th International Conference on Intelligent Human-Machine Systems and Cybernetics (IHMSC), 26-27 Aug. 2013, Hangzhou, China, vol. 1, pp. 172-176.

[184] X. Hu, Y. Shi, and R. C. Eberhart, "Recent advances in particle swarm," in Proc. IEEE Congress on Evolutionary Computation 2004. Vancouver, BC, Canada, 24-29 Jul. 2016, vol. 1, pp. 90-97.

[185] J. Han, H. Cheng, D. Xin, and X. Yan, "Frequent pattern mining: current status and future directions," Data Mining and Knowledge Discovery, vol. 15 , no. 1, pp. 55-86, 2007.

[186] J. Han, G. Dong, and Y. Yin, "Efficient mining of partial periodic patterns in time series database," in Proc. 15th International Conference on Data Engineering, Mar. 23-26, 1999, Sydney, Australia, pp. 106115.

[187] Z. Wen, C. Fan, X. Zhang, Y. Wu, J. Zou, and J. Liu, "A learning spectrum hole prediction model for cognitive radio systems," in Proc. 10th IEEE International Conference on Computer and Information Technology (CIT), Bradford, UK, 29 Jun.-1 Jul. 2010, pp. 2089-2093.

[188] H. Kim and K. G. Shin, "Optimal online sensing sequence in multichannel cognitive radio networks," IEEE Trans. Mobile Comput., vol. 12, no. 7, pp. 1349-1362, 2013.

[189] N. I. Sapankevych and R. Sankar, "Time series prediction using support vector machines: A survey," IEEE Comput. Intell. Mag., vol. 4, no. 2, pp. 24-38, 2009.

[190] S. Ni and S. Shen, "Frequency spectrum access mechanism of cognitive radio based on spectrum prediction," in Proc. IET International Conference on Communication Technology and Application (ICCTA), 14-16 Oct. 2011, Beijing, China. pp. 556-560.

[191] C.-J. Yu, Y.-Y. He, and T.-F. Quan, "Frequency spectrum prediction method based on EMD and SVR," in Proc. 8th International Conference on Intelligent Systems Design and Applications, 26-28 Nov. 2008, Kaohsiung, Taiwan, vol. 3, pp. 39-44.

[192] S. Yin, D. Chen, Q. Zhang, and S. Li, "Prediction-based throughput optimization for dynamic spectrum access," IEEE Trans. Veh. Technol., vol. 60, no. 3, pp. 1284-1289, 2011.

[193] H. Li, "Reconstructing spectrum occupancies for wideband cognitive radio networks: A matrix completion via belief propagation," in Proc. IEEE International Conference on Communications (ICC), 23-27 May 2010, Cape Town, South Africa, pp. 1-6.

[194] S. Arkoulis, E. Anifantis, V. Karyotis, S. Papavassiliou, and N. Mitrou, "Discovering and exploiting spectrum power correlations in cognitive radio networks: an experimentally driven approach," EURASIP Journal on Wireless Communications and Networking, vol. 2014, no. 1, p. 17, 2014.

[195] K. Hossain, B. Champagne, and A. Assra, "Cooperative multiband joint detection with correlated spectral occupancy in cognitive radio networks," IEEE Trans. Signal Process., vol. 60, no. 5, pp. 2682-2687, 2012.

[196] K. Hossain and B. Champagne, "Wideband spectrum sensing for cognitive radios with correlated subband occupancy," IEEE Signal Process. Lett., vol. 18, no. 1, pp. 35-38, 2011.

[197] J. Pearl, Probabilistic reasoning in intelligent systems: networks of plausible inference. Morgan Kaufmann, 1988.

[198] H. Li and R. C. Qiu, "A graphical framework for spectrum modeling and decision making in cognitive radio networks," in Proc. of the IEEE Global Commun. Conf (GLOBECOM), 6-10 Dec. 2010, Miami, Florida, USA.

[199] Z. Qin, et al, "Link quality analysis-based channel selection in HF asynchronous automatic link establishment: A matrix completion approach," to appear in IEEE System Journal, 2017.

[200] S.-J. Kim and G. B. Giannakis, "Cognitive radio spectrum prediction using dictionary learning," in Proc. of the IEEE Global Commun. Conf (GLOBECOM), 9-13 Dec. 2013, Atlanta, Georgia, USA. pp. 1-6.

[201] Y. Wang, Z. Z. Zhang, Q. Y. Yu, and J. M. Chen, "A novel predictionbased spectrum allocation mechanism for mobile cognitive radio networks," KSII Transactions on Internet and Information Systems, vol. 7, no. 9, pp. 2101-2119, 2013.

[202] Y. Wang, Z. Zhang, L. Ma, and J. Chen, "SVM-Based spectrum mobility prediction scheme in mobile cognitive radio networks," The Scientific World Journal, vol. 2014.

[203] J. Guo, H. Ji, Y. Li, and X. Li, "A novel spectrum handoff management scheme based on svm in cognitive radio networks," in Proc. 6th International ICST Conference on Communications and Networking in China (CHINACOM), 17-19 Aug. 2011, Harbin, China, pp. 645-649.
[204] L. Hou, K.-H. Yeung, and K.-Y. Wong, "Link availability prediction for cognitive radio Ad Hoc networks," Journal of Computer and Communications, vol. 2014.

[205] I. Butun, A. Cagatay Talay, D. T. Altilar, M. Khalid, and R. Sankar, "Impact of mobility prediction on the performance of cognitive radio networks," in Proc. Wireless Telecommunications Symposium (WTS) 21-23 Apr. 2010, Tampa, FL, USA, pp. 1-5.

[206] Z. Wang and S. Salous, "Time series ARIMA model of spectrum occupancy for cognitive radio," in Proc. IET Seminar on Cognitive Radio and Software Defined Radios: Technologies and Techniques, 18 Sep. 2008, London, UK, pp. 1-4.

[207] S. Zhiyuan, J. Xueyuan, and H. Lianfen, "A prediction approach for MAC layer sensing in cognitive radio networks," in Proc. IET 2nd International Conference on Wireless, Mobile and Multimedia Networks (ICWMMN), 12-15 Oct. 2008, Beijing, China, pp. 317-320.

[208] B. Houlding, A. Bhattacharya, S. P. Wilson, and T. K. Forde, "A fast Bayesian model for latent radio signal prediction," in Proc. 7th International Symposium on Modeling and Optimization in Mobile, Ad Hoc, and Wireless Networks, 2009, Seoul, Korea (South), pp. 1-7.

[209] G. Ding, F. Wu, Q. Wu, et al, "Robust online spectrum prediction with incomplete and corrupted historical observations," to appear in IEEE Transactions on Vehicular Technology, 2017.

[210] G. Ding, J. Wang, Q. Wu, L. Yu, Y. Jiao, and X. Gao, "Joint spectraltemporal spectrum prediction from incomplete historical observations," in Proc. IEEE Global Conference on Signal and Information Processing (GLOBALSIP), 3-5 Dec. 2014, Atlanta, Georgia, USA, pp. 1-6.

[211] G. Ding, S. Zhai, X. Chen, Y. Zhang, and C. Liu, "Robust spectraltemporal two-dimensional spectrum prediction," in Proc. EAI International Conference on Machine Learning and Intelligent Communications (MLICOM), 26-28 Aug. 2016, Shanghai, China, pp. 1-9.

[212] M. Tang, Z. Zheng, G. Ding, and Z. Xue, "Efficient TV white space database construction via spectrum sensing and spatial inference," in Proc. IEEE International Performance Computing and Communications conference (IPCCC), 14-16 Dec. 2015. Nanjing, China, pp. 1-6.

[213] M. Tang, G. Ding, Q. Wu, Z. Xue, and T. A. Tsiftsis, "A joint tensor completion and prediction scheme for multi-dimensional spectrum map construction," IEEE Access, vol. 4, pp. 8044-8052, Nov. 2016.

[214] Q. Wu, G. Ding, Z. Du, Y. Sun, M. Jo, and A. V. Vasilakos, "A cloudbased architecture for the Internet of spectrum devices (IoSD) over future wireless networks," IEEE Access, vol. 4, pp. 4247-4255, Jun. 2016.

[215] J. Qiu, Q. Wu, G. Ding, Y. Xu, and S. Feng, "A survey of machine learning for big data processing," EURASIP Journal on Advances in Signal Processing, vol. 2016, no. 81, May 2016.

[216] P. Huang, C.-J. Liu, L. Xiao, and J. Chen, "Mining frequent partial periodic patterns in spectrum usage data," in Proc. of the 2012 IEEE 20th International Workshop on Quality of Service, 4-5 Jun. 2012, Coimbra, Portugal, pp. 1-4.

[217] M. T. Ozden, "Adaptive multichannel sequential lattice prediction filtering method for ARMA spectrum estimation in subbands," EURASIP Journal on Advances in Signal Processing, vol. 2013, no. 1, pp. 1-36, 2013.

[218] D. W. Browne, "Predicting communications activity in the radio spectrum," in Proc. Conference Record of the Forty Sixth Asilomar Conference on Signals, Systems and Computers (ASILOMAR), 4-7 Nov. 2012, Pacific Grove, California, USA, pp. 1069-1073.

[219] J. Qadir, "Artificial intelligence based cognitive routing for cognitive radio networks," Artificial Intelligence Review, volu. 45, no. 1, pp. 25 96, Jan. 2016.

[220] Z. Wang and S. Salous, "Spectrum occupancy statistics and time series models for cognitive radio," Journal of Signal Processing Systems, vol. 62, no. 2, pp. 145-155, 2011.

[221] S. D. Barnes and B. Maharaj, "Prediction based channel allocation performance for cognitive radio," AEU-International Journal of Electronics and Communications, vol. 68, no. 4, pp. 336-345, 2014.

[222] N. Guoqin, D. Jiaqi, S. Jian, and Q. Duo, "Spectrum sharing based on spectrum heterogeneity and multi-hop handoff in centralized cognitive radio networks," in Proc. Wireless and Optical Communications Conference (WOCC), 15-16 Apr. 2011, Newark, NJ, USA, pp. 1-6.

[223] C. Xu, Y. Li, Y. Yang, and Y. Xian, "A novel spectrum prediction algorithm for cognitive radio system based on chaotic neural network?" Journal of Computational Information Systems, vol. 9, no. 1, pp. 313320, 2013.

[224] Q.-L. Ma, Q.-L. Zheng, H. Peng, T.-W. Zhong, and L.-Q. Xu, "Chaotic time series prediction based on evolving recurrent neural networks," in Proc. International Conference on Machine Learning and Cybernetics, Aug. 2007, Hong Kong, vol. 6, pp. 3496-3500. 
[225] Z. Yang, L. Yang, and Y. Fu, "Spectrum prediction for high-frequency radar based on extreme learning machine," in Proc. 7th International Conference on Advanced Computational Intelligence, 27-29 Mar. 2015, Fujian, China, pp. 235-239.

[226] S. Bai, X. Zhou, and F. Xu, "Spectrum prediction based on improvedback-propagation neural networks," in Proc. 11th International Conference on Natural Computation (ICNC), 15-17 Aug. 2015, Zhangjiajie, China, pp. 1006-1011.

[227] Z. Zhang, K. Zhang, F. Gao, and S. Zhang, "Spectrum prediction and channel selection for sensing-based spectrum sharing scheme using online learning techniques," in Proc. 26th Annual International Symposium on Personal, Indoor, and Mobile Radio Communications (PIMRC), Aug. 30-Sep. 2 2015, Hong Kong, China, pp. 355-359.

[228] H. Eltom, S. Kandeepan, B. Moran, and R. Evans, "Spectrum occupancy prediction using a hidden Markov model," in Proc. International Conference on Signal Processing and Communication Systems (ICSPCS), 14-16 Dec.2015, Cairns, Australia, pp. 1-8.

[229] A. Eltholth, "Forward backward autoregressive spectrum prediction scheme in cognitive radio network," in Proc. 9th International Conference on Signal Processing and Commuication Systems (ICSPCS), 14-16 Dec. 2015, Cairns, Australia, pp. 1-5.

[230] Y. Zhao, Z. Hong, G. Wang, and J. Huang, "High-order hidden bivariate Markov model-a novel approach on spectrum prediction," in Proc. 25th International Conference on Computer Communication and Networks (ICCCN), 1-4 Aug. 2016, Waikoloa, Hawaii, USA, pp. 1-7.

[231] H. Eltom, S. Kandeepan, Y. Liang, B. Moran, and R. Evans, "HMM based cooperative spectrum occupancy prediction using hard fusion," in Proc. IEEE International Conference on Communications Workshops (ICC), 23-27 May 2016, Kuala Lumpur, Malaysia, pp. 669-675.

[232] A. Eltholth, "Spectrum prediction in cognitive radio systems using a wavelet neural network," in Proc. 24th International Conference on Software, Telecommunications and Computer Networks (SoftOOM), 2224 Sep. 2016, Split, Croatia, pp. 1-6.

[233] A. Saad, B. Staehle, and R. Knorr, "Spectrum prediction using hidden Markov models for industrial cognitive radio," in Proc. IEEE 12th International Conference on Wireless and Mobile Computing, Networking and Communications (WiMob), 17-19 Oct. 2016, New York, USA, pp. $1-7$.

[234] F. Hou, X. Chen, H. Huang, and X. Jing, "Throughput performance improvement in cognitive radio networks based on spectrum prediction," in Proc. 6th International Symposium on Communications and Information Technologies (ISCIT), 26-28 Sep. 2016, Qingdao, China, pp. 655-658.

[235] M. Huk and J. Mizera-Pietraszko, "Contextual neural-network based spectrum prediction for cognitive radio," in Proc. Fourth International Conference on Future Generation Communication Technology (FGCT), 29-31 July 2015, London, UK, pp. 1-5.

[236] L. Yu, J. Chen, G. Ding, Z. Qin, "Fast automatic link establishment: A new metric and the value of spectrum prediction," in Proc. 7th Wireless Communications \& Signal Processing (WCSP), Oct. 2016.

[237] J. Zhang, G. Ding, Y. Xu, and F. Song, "On the usefulness of spectrum prediction for dynamic spectrum access," in Proc. 7th Wireless Communications \& Signal Processing (WCSP), Oct. 2016.

[238] M. Tang, G. Ding, Z. Xue, J. Zhang, and H. Zhou, "Multi-dimensional spectrum map construction: A tensor perspective," in Proc. 7th Wireless Communications \& Signal Processing (WCSP), Oct. 2016.

[239] K.-M. Kang et al., "Deployment and coverage of cognitive radio networks in TV white space," IEEE Commun. Mag., vol. 50, no. 12, pp. 88C94, Dec. 2012.

[240] FCC, FCC Adopt Rule for Unlicensed Use of Television White Spaces. [Online]. Available: http://www.fcc.gov

[241] FCC, Office of Engineering and Technology Authorizes TV White Space Database Administrators to Provide Service to Unlicensed Devices Operating on Unused TV Spectrum Nationwide, ET Docket No. 04186, Mar. 2013.

[242] R. Murty, R. Chandra, T. Moscibroda, and P. Bahl, "Senseless: A database-driven white spaces network," IEEE Trans. Mobile Comput., vol. 11, no. 2, pp. 189C203, Feb. 2012

[243] ETSI, "System architecture for information exchange between different geo-location databases (GLDBs) enabling the operation of white space devices (WSDs)," Tech. Rep., 2015. http://www.etsi.org/deliver

[244] CSMAC Committee, "Interference and dynamic spectrum access," National Telecommunications and Information Administration (NTIA), USA, Tech. Rep., November, 2010. http: //www.ntia.doc.gov/legacy/advisory/spectrum/reports

[245] Institute for Telecommunication Sciences, "Developing forward thinking rules and processes to fully exploit spectrum resources: Case study exploring approaches for real-time federal spectrum sharing," in Proc. 13th Annual International Symposium on Advanced Radio Technologies, 24-26 July 2012, Boulder, Colorado, NTIA Special Publication SP-14-508.

[246] J. Wang, G. Ding, Q. Wu, L. Shen, and F. Song, "Spatial-temporal spectrum hole discovery: A hybrid spectrum sensing and geolocation database framework," Chin. Sci. Bull., vol. 59, no. 16, pp. 1896C1902, Jun. 2014.

[247] SpectrumBridge. [Online]. Available: http://whitespaces.spectrumbridge. com

[248] Google Spectrum Database. [Online]. Available: http://www.google.org/spectrum/whitespace/

[249] Microsoft Research http://whitespaces.msresearch.us/WSWebGUI/whitespaces.aspx

[250] Telcordia. White Space Database-Capitalize on Dynamic Spectrum Access Opportunities. [Online]. Available: http$\mathrm{s}: / /$ prism.telcordia.com/tvws/main/home/index.shtml

[251] Y. Zhao, J. Gaeddert, K. K. Bae, and J. H. Reed, "Radio environment map enabled situation-aware cognitive radio learning algorithms," in Proc. Software Defined Radio Forum (SDRF) Technical Conference, 2006.

[252] Y. Zhao, L. Morales, J. Gaeddert, K. K. Bae, J. S. Um, and J. H. Reed, "Applying radio environment maps to cognitive wireless regional area networks," In Proc. 2nd IEEE Intl. Symposium on New Frontiers in Dynamic Spectrum Access Networks (DySPAN 2007), 17-20 April 2007, Dublin, Ireland.

[253] Y. Zhao, "Enabling cognitive radios through radio environment maps," Ph.D. dissertation, Virginia Polytechnic Institute and State University, 2007.

[254] H. B. Yilmaz, T. Tugcu, F. Alagoz, and S. Bayhan, "Radio environment map as enabler for practical cognitive radio networks," IEEE Commun. Mag., vol. 51, no. 12, pp. 162-169, 2013.

[255] J. Perez-Romero, A. Zalonis, L. Boukhatem, A. Kliks, K. Koutlia, N. Dimitriou, and R. Kurda, "On the use of radio environment maps for interference management in heterogeneous networks," IEEE Commun. Mag., vol. 53, no. 8, pp. 184-191, 2015.

[256] S. Debroy, S. Bhattacharjee, and M. Chatterjee, "Spectrum map and its application in resource management in cognitive radio networks," IEEE Transactions on Cognitive Communications and Networking, vol. 1, no. 4, pp. 406-419, 2016.

[257] Berna Sayrac, Sana Ben Berna Sayrac, Sana Ben Jemaa \& Pascal \& Pascal Cordier Cordier Orange Labs, "Radio environmental maps (REMs): A cognitive tool for environmental awareness, Tech. Rep., http://www-syscom.univ-mlv.fr/ najim/gdr-ecoradio/sayrac.pdf.

[258] Z. Quan, S. Cui, and A. H. Sayed, "Optimal linear cooperation for spectrum sensing in cognitive radio networks," IEEE J. Sel. Topics Signal Process., vol. 2, no. 1, pp. 28-40, 2008.

[259] J. G. Andrews, S. Buzzi, W. Choi, S. V. Hanly, A. Lozano, A. C. Soong, and J. C. Zhang, "What will 5G be?" IEEE J. Sel. Areas Commun., vol. 32, no. 6, pp. 1065 - 1082, 2014.

[260] J. Zhao and J. Wang, "Joint optimization algorithm based on centralized spectrum sharing for cognitive radio,"in Proc. IEEE International Conference on Communications (ICC), 8-12 Jun. 2015, London, UK, pp. 7653-7658.

[261] J. Mitola III, J. Guerci, J. Reed, Y.-D. Yao, Y. Chen, T. C. Clancy, J. Dwyer, H. Li, H. Man, and R. McGwier, "Accelerating 5G QoE via public-private spectrum sharing," IEEE Commun. Mag., vol. 52, no. 5, pp. 77-85, 2014

[262] E. E. Johnson, S. Cook, R. I. Desourdis, G. Earle, and J. C. Ostergaard, Advanced High-Frequency Radio Communications. Artech House, Inc., 1997.

[263] E. E. Johnson, E. Koski, W. N. Furman, M. Jorgenson, and J. Nieto, Third-generation and Wideband HF Radio Communications. Artech House, 2012

[264] J. Wang, Research and Development of HF Digital Communications (in Chinese). Beijing: Science Press, 2013.

[265] E. Koski and W. Furman, "Applying cognitive radio concepts to HF communications," in Proc. 11th International Conference on Ionospheric Radio Systems and Techniques (IRST), 2009, Edinburgh, Scotland, United Kingdom, pp. 1-6.

[266] W. Furman and E. Koski, "Next generation ALE concepts," Proc. 11th International Conference on Ionospheric Radio Systems and Techniques (IRST), 2009, Edinburgh, Scotland, United Kingdom, pp. $1-5$.

[267] A. Shahid, S. Ahmad, A. Akram, and S. A. Khan, "Cognitive ALE for HF radios," in Proc. Second International Conference on Computer 
Engineering and Applications (ICCEA), 19-21 Mar. 2010, Bali Island, Indonesia, vol. 2, pp. 28-33.

[268] W. Furman, E. Koski, and J. Nieto, "Design concepts for a wideband HF ALE capability," in Proc. 12th IET International Conference on Ionospheric Radio Systems and Techniques (IRST), 15-17 May 2012, York, UK, pp. 1-5.

[269] A. A. Khan, M. H. Rehmani, and M. Reisslein, "Cognitive radio for smart grids: Survey of architectures, spectrum sensing mechanisms, and networking protocols," IEEE Commun. Surveys Tuts., vol. 18, no. 1, pp. 860-898, Firstquarter 2016.

[270] R. C. Qiu, et al., "Cognitive radio network for the smart grid: Experimental system architecture, control algorithms, security, and microgrid testbed," IEEE Transactions on Smart Grid vol. 2, no. 4 pp. 724-740, 2011.

[271] O. B. Akan, O. B. Karli, O. Ergul, "Cognitive radio sensor networks," IEEE Network, vol. 23, no.4, pp. 34-40, July 2009.

[272] A. O. Bicen, V. C. Gungor, O. B. Akan, "Delay-sensitive and multimedia communication in cognitive radio sensor networks," Ad Hoc Networks Journal (Elsevier), vol. 10, no. 5, pp. 816-830, July 2012.

[273] M. Ozger, E. A. Fadel, O. B. Akan, "Event-to-sink spectrum-aware clustering in mobile cognitive radio sensor networks," IEEE Trans. Mobile Comput., vol. 15, no. 9, pp. 2221-2233, 2016.

[274] S. H. R. Bukhari, M. H. Rehmani, and S. Siraj, "A survey of channel bonding for wireless networks and guidelines of channel bonding for futuristic cognitive radio sensor networks," IEEE Commun. Surveys Tuts., vol. 18, no. 2, pp. 924-948, Secondquarter 2016.

[275] H. ElSawy, E. Hossain, and M. Haenggi, "Stochastic geometry for modeling, analysis, and design of multi-tier and cognitive cellular wireless networks: A survey," IEEE Commun. Surveys Tuts., vol. 15, pp. 996-1019, July 2013.

[276] M. Chen, W. Saad, and C. Yin, "Optimized uplink-downlink decoupling in LTE-U networks: An Echo State Approach," in Proc. IEEE International Conference on Communications (ICC), Mobile and Wireless Networks Symposium, 22-27 May 2016, Kualalumpur, Malaysia, pp. $1-6$

[277] M. Chen, W. Saad, C. Yin, and M. Debbah, "Echo state networks for proactive caching and content prediction in cloud radio access networks," in Proc. IEEE Global Communications Conference (GLOBECOM), Workshop on 5G RAN Design, 4-8 Dec. 2016, Washington, DC, USA, pp. 1-6.

[278] Y. Zhang, R. Yu, M. Nekovee, Y. Liu, S. Xie, and S. Gjessing, "Cognitive machine-to-machine communications: Visions and potentials for the smart grid," IEEE Network, vol. 26, no. 3, pp. 6-13, 2012.

[279] A. McAfee, E. Brynjolfsson, T. H. Davenport, D. Patil, and D. Barton, "Big data," The Management Revolution. Harvard Bus. Rev., vol. 90, no. 10, pp. 61-67, 2012.

[280] W.-B. Chien, C.-K. Yang, and Y.-H. Huang, "Energy-saving cooperative spectrum sensing processor for cognitive radio system," IEEE Trans. Circuits Syst. I, Reg. Papers, vol. 58, no. 4, pp. 711-723, 2011.

[281] T. Jing, X. Xing, W. Cheng, Y. Huo, and T. Znati, "Cooperative spectrum prediction in multi-PU multi-SU cognitive radio networks," in Proc. 8th International Conference on Cognitive Radio Oriented Wireless Networks (CROWNCOM), 8-10 July 2013, Washington DC, United States, pp. 25-30.

[282] R. Umar and A. U. H. Sheikh, "A comparative study of spectrum awareness techniques for cognitive radio oriented wireless networks," Physical Communication, vol. 9, pp. 148-170, 2013.

[283] T. O'Shea and C. Clancy, "Deep reinforcement learning radio control and signal detection with KeRLym, a Gym RL agent," arXiv preprint, arXiv:1605.09221, 2016.

[284] R. McGwier, T. O'Shea, K. Karra, M. Fowler, "Recent developments in artificial intelligence applications of deep learning for signal processing," Virginia Tech Wireless Symposium, 2016.

[285] F. H. Sanders, R. L. Hinkle, and B. J. Ramsey, "Measurement procedures for the radar spectrum engineering criteria (RSEC)," 2005 Available: https://www.its.bldrdoc.gov/publications/download /TR-05-420.pdf

[286] F. H. Sanders, E. F. Drocella, and R. L. Sole, "Using on-shore detected radar signal power for interference protection of off-shore radar receivers," National Telecommunications and Information Administration (NTIA), USA, Tech. Rep., March, 2016, [Online]. Available:https://www.its.bldrdoc.gov/publications/ download/TR-16-521.pdf

[287] Z. Khan, J. J. Lehtomaki, S. I. Iellamo, R. Vuohtoniemi, E. Hossain, and Z. Han, "IoT connectivity in radar bands: A shared access model based on spectrum measurements," IEEE Communications Magazine, vol. 55 , no. 2 , pp. $88-96,2017$
[288] S. Bhattarai, J. M. J. Park, B. Gao, K. Bian, and W. Lehr, "An overview of dynamic spectrum sharing: Ongoing initiatives, challenges, and a roadmap for future research," IEEE Transactions on Cognitive Communications and Networking, vol. 2, no. 2, pp. 110-128, 2016.

[289] F. Hessar and S. Roy, "Spectrum sharing between a surveillance radar and secondary WiFi networks," IEEE Transactions on Aerospace and Electronic Systems, vol. 52, no. 3, pp. 1434-1448, June 2016.

[290] S. W. Boyd et al, "Spectrum monitoring during reception in dynamic spectrum access cognitive radio networks," IEEE Trans. Commun., vol. 60, no. 2, pp. 547-558, Feb. 2012.

[291] A. Ali and W. Hamouda, "Spectrum monitoring using energy ratio algorithm for OFDM-based cognitive radio networks," IEEE Trans. Wireless Commun., vol. 14, no. 4, pp. 2257-2268, Apr. 2015.

[292] T. Ihalainen et al, "Spectrum monitoring scheme for filter bank based cognitive radios," in Proc. Future Network and Mobile Summit, 16-18 June 2010, Florence, Italy, pp. 1-6.

[293] C. Song, Z. Qu, N. Blumm, and A.-L. Barabasi, "Limits of predictability in human mobility," Science, vol. 327, no. 5968, pp. 1018-1021, Feb. 2010.

[294] http://orca.feit.ukim.edu.mk/index.html.

[295] O. Fratu, et al, "Comparative study of radio mobile and ICS telecom propagation prediction models for DVB-T," IEEE International Symposium on Broadband Multimedia Systems and Broadcasting (BMSB), pp. 1-6, 17-19 June 2015 .

[296] P. Lazaridis, et al, "Evaluation of prediction accuracy for the LongleyRice model in the FM and TV bands," IX international Conference EATI-2013, Ohrid, Macedonia, 26-28 Sep. 2013.

[297] http://www.wishful-project.eu/.

[298] http://www.crew-project.eu/.

[299] M. Pesko, et al, "The indirect self-tuning method for constructing radio environment map using omnidirectional or directional transmitter antenna," EURASIP Journal on Wireless Communications and Networking, vol. 2015, no. 1. pp. 1-12, Dec. 2015.

[300] http://www.ict-faramir.eu.

[301] J. Riihijarvi, J. Nasreddine, and P. Mahonen, "Demonstrating radio environment map construction from massive data sets," Proc. IEEE DySPAN, Bellevue, WA, USA, Oct. 2012, pp. 266-267.

[302] A. Palaios, et al, "Measurements of spectrum use in London: Exploratory data analysis and study of temporal, spatial and frequency-domain dynamics," Proc. IEEE DySPAN, Bellevue, WA, USA, Oct. 2012, pp. 154-165.

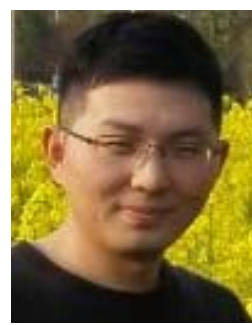

Guoru Ding (S'10-M'14-SM'16) received his B.S. degree (Hons.) in electrical engineering from Xidian University, Xi' an, China, in 2008 and his Ph.D. degree (Hons.) in communications and information systems in College of Communications Engineering, Nanjing, China, in 2014. Since 2014, he has been an assistant professor in College of Communications Engineering and a research fellow in National High Frequency Communications Research Center of China. Since April 2015, he has been a Postdoctoral Research Associate at the National Mobile Communications Research Laboratory, Southeast University, Nanjing, China. His research interests include cognitive radio networks, massive MIMO, machine learning, and big data analytics over wireless networks.

He has served as a Guest Editor of the IEEE Journal on SElectED AREaS in COMMUNiCATIONS (Special issue on spectrum sharing and aggregation in future wireless networks). He is now an Associate Editor of THE JOURNAL OF COMMUNICATIONS AND INFORMATION NETWORKS, THE KSII TRANSACTIONS ON INTERNET AND INFORMATION SYSTEMS and THE AEU-INTERNATIONAL JOURNAL OF ELECTRONICS AND COMMUNICATIONS. He has acted as Technical Program Committees (TPC) members for a number of international conferences, including the IEEE Global Communications Conference (GLOBECOM), IEEE International Conference on Communications (ICC), and IEEE Vehicular Technology Conference (VTC). $\mathrm{He}$ is a Voting Member of the IEEE 1900.6 Standard Association Working Group. He was a recipient of the Best Paper Awards from EAI MLICOM 2016, IEEE VTC 2014-Fall, and IEEE WCSP 2009. He was awarded the Alexander von Humboldt Fellowship in 2017 and the Excellent Doctoral Thesis Award of China Institute of Communications in 2016. 


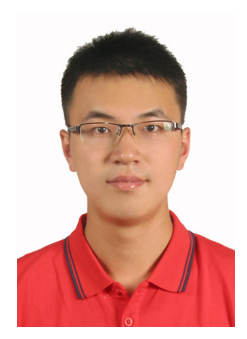

Yutao Jiao is currently a Ph.D. student in the School of Computer Science and Engineering, Nanyang Technological University. He received a B.S. degree from the College of Communications Engineering, Nanjing, China, in 2013. His research interests include resource management in the Internet of Things and economics of big data.

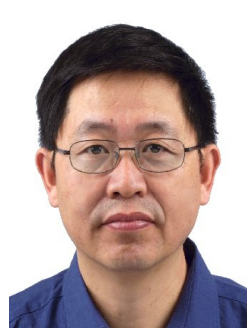

Jinlong Wang is currently a Professor at PLA University of Science and Technology, Nanjing, China. $\mathrm{He}$ received the B.S. degree in wireless communications, M.S. degree and Ph.D. degree in communications and electronic systems from Institute of Communications Engineering, Nanjing, China, in 1983, 1986 and 1992, respectively.

Dr. Wang is the co-chair of IEEE Nanjing Section. His current research interests span a wide range of topics in wireless communications and signal processing, including cognitive radio networks, HF communications, cooperative communications, and wireless security. In these areas, he has published extensively in internationally renowned journals, including the IEEE Communications Magazine, IEEE Signal Processing Magazine, IEEE Transactions on Communications, IEEE Transactions on Wireless Communications, and IEEE Transactions on Vehicular Technology, etc.

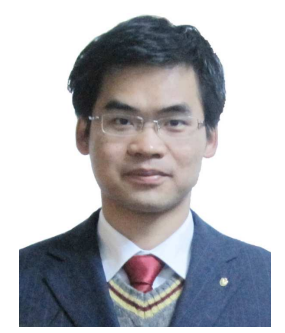

Yulong Zou (S'07-M'12-SM'13) is a Full Professor at the Nanjing University of Posts and Telecommunications (NUPT), Nanjing, China. He received the B.Eng. degree in information engineering from NUPT, Nanjing, China, in July 2006, the first Ph.D. degree from the Stevens Institute of Technology, New Jersey, United States, in May 2012, and the second Ph.D. degree from NUPT, Nanjing, China, in July 2012.

Dr. Zou is currently serving as an editor for the IEEE Communications Surveys \& Tutorials, IEEE Communications Letters, EURASIP Journal on Advances in Signal Processing, and KSII Transactions on Internet and Information Systems. He is also serving as a lead guest editor for special issue on Security Challenges and Issues in Cognitive Radio Networks in the EURASIP Journal on Advances in Signal Processing. In addition, he has acted as symposium chairs, session chairs, and TPC members for a number of IEEE sponsored conferences, including the IEEE Wireless Communications and Networking Conference (WCNC), IEEE Global Telecommunications Conference (GLOBECOM), IEEE International Conference on Communications (ICC), IEEE Vehicular Technology Conference (VTC), International Conference on Communications in China (ICCC), International Conference on Communications and Networking in China (ChinaCom) and so on. His research interests span a wide range of topics in wireless communications and signal processing, including the cooperative communications, cognitive radio, wireless security, and green communications. In these areas, he has published extensively in internationally renowned journals, including the IEEE Transactions on Signal Processing, IEEE Transactions on Communications, IEEE Journal on Selected Areas in Communications, IEEE Transactions on Wireless Communications, IEEE Transactions on Vehicular Technology, and IEEE Communications Magazine.

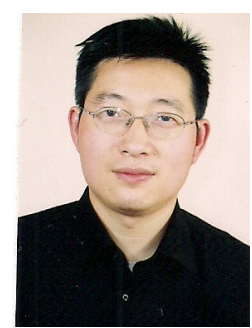

Qihui Wu (SM'13) received his B.S. degree in communications engineering, M.S. degree and Ph.D. degree in communications and information systems from Institute of Communications Engineering, Nanjing, China, in 1994, 1997 and 2000, respectively. From 2003 to 2005, he was a Postdoctoral Research Associate at Southeast University, Nanjing, China. From 2005 to 2007, he was an Associate Professor with the College of Communications Engineering, PLA University of Science and Technology, Nanjing, China, where he served as a Full Professor from 2008 to 2016. Since May 2016, he has been a full professor with the College of Electronic and Information Engineering, Nanjing University of Aeronautics and Astronautics, Nanjing, China. From March 2011 to September 2011, he was an Advanced Visiting Scholar in Stevens Institute of Technology, Hoboken, USA. Dr. Wu's current research interests span the areas of wireless communications and statistical signal processing, with emphasis on system design of software defined radio, cognitive radio, and smart radio.

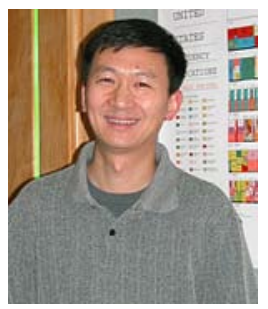

Yu-Dong Yao (S'88-M'88-SM'94-F'11) is currently a professor and department director of electrical and computer engineering. He is also a director of Stevens Wireless Information Systems Engineering Laboratory (WISELAB). He has been with Stevens Institute of Technology, Hoboken, New Jersey, since 2000. Previously, from 1989 and 1990, he was at Carleton University, Ottawa, Canada, as a Research Associate working on mobile radio communications. From 1990 to 1994, he was with Spar Aerospace Ltd., Montreal, Canada, where he was involved in research on satellite communications. From 1994 to 2000, he was with Qualcomm Inc., San Diego, CA, where he participated in research and development in wireless code-division multiple-access (CDMA) systems.

$\mathrm{He}$ holds one Chinese patent and twelve U.S. patents. His research interests include wireless communications and networks, spread spectrum and CDMA, antenna arrays and beamforming, cognitive and software defined radio (CSDR), and digital signal processing for wireless systems. Dr. Yao was an Associate Editor of IEEE Communications Letters and IEEE Transactions on Vehicular Technology, and an Editor for IEEE Transactions on Wireless Communications. He received the B.Eng. and M.Eng. degrees from Nanjing University of Posts and Telecommunications, Nanjing, China, in 1982 and 1985, respectively, and the Ph.D. degree from Southeast University, Nanjing, China, in 1988, all in electrical engineering. 


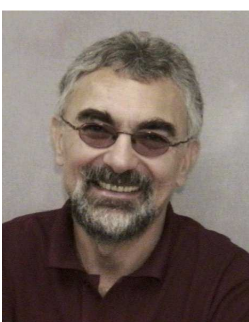

Lajos Hanzo received his Masters degree in electronics in 1976 and his Doctorate in 1983 from the Technical University of Budapest. In 2010 he was awarded the universitys highest honour, namely the Honorary Doctorate Doctor Honaris Causa. Since 1986 he has been with the University of Southampton, UK and in 2004 he was awarded the Doctor of Sciences (DSc) degree. During his 36-year career in telecommunications he has held various research and academic posts in Hungary, Germany and the UK. Since 1986 he has been a member of academic staff in the School of Electronics and Computer Science, University of Southampton, UK, where he currently holds the Chair in Telecommunications and he is head of the Communications Research Area. He is also a Chaired Professor at Tsinghua University, Beijing, China.

Lajos Hanzo has co-authored 20 John Wiley/IEEE Press books totalling about 10000 pages on mobile radio communications, and published $1200+$ research papers and book chapters at IEEE Xplore. He has also organised and chaired major IEEE conferences, such as WCNC2006, WCNC2009, VTC2011, presented Tutorial/overview lectures at international conferences. He presented a number of named lectures and keynotes. Lajos is also an IEEE Distinguished Lecturer of both the Communications Society and the Vehicular Society as well as a Fellow of both the IEEE and the IEE/IET, Fellow of the Royal Academy of Engineering (FREng). He is acting as a Governor of the IEEE VTS as well as of ComSoc. Lajos is the Editorin-Chief of the IEEE Press. He has been awarded a number of distinctions, most recently the IEEE Wireless Technical Committee Achievement Award (2007), the IET Sir Monti Finniston Achievement Award across all disciplines of engineering (2008) and an Honorary Doctorate of the Technical University of Budapest (2010). His most recent paper awards are: WCNC2007 in Hong Kong, ICC'2009 Dresden and ICC'2010 Cape Town. 